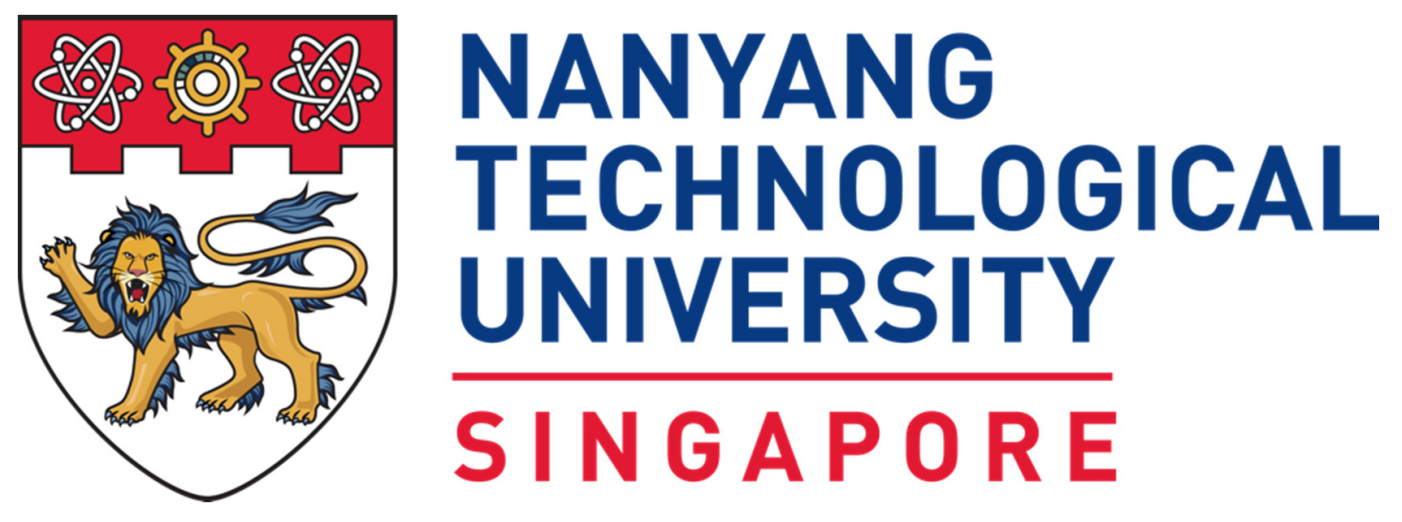

FABRICATION AND CHARACTERIZATION OF YTTERBIUM DOPED TRANSPARENT LASER CERAMICS

LUO DEWEI

SCHOOL OF MATERIALS SCIENCE AND ENGINEERING 



\title{
FABRICATION AND CHARACTERIZATION OF YTTERBIUM DOPED TRANSPARENT LASER CERAMICS
}

\author{
LUO DEWEI
}

SCHOOL OF MATERIALS SCIENCE AND ENGINEERING

A thesis submitted to the Nanyang Technological University in partial fulfillment of the requirement for the degree of Doctor of Philosophy 



\section{Statement of Originality}

I hereby certify that the work embodied in this thesis is the result of original research and has not been submitted for a higher degree to any other University or Institution.

$1 / 11 / 2017$

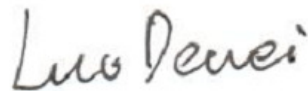

Date

Luo Dewei 



\begin{abstract}
Transparent laser ceramics have shown great application potentials as the host materials of solid state lasers, because of their excellent physical and stable chemical properties. Solid state lasers have wide applications in the fields of industry, communication, medical and military, such as laser drilling and welding, laser surgery and even laser weapons. Compared with their single crystal counterparts, polycrystalline ceramics possess various advantages, such as shorter fabrication period, higher yield for mass production, higher ion doping concentration, feasibility to be large sizes with complex shapes and structures, and overall lower fabrication cost.
\end{abstract}

Among various laser ceramics, ytterbium $\left(\mathrm{Yb}^{3+}\right)$ ion doped ones have been considered to be very attractive solid state laser materials. $\mathrm{Yb}^{3+}$ ion has unique properties, including high quantum efficiency, long fluorescence lifetime and broad emission spectrum. Its broad absorption band is especially useful for direct laser diode pumping. Yttrium aluminum garnet (YAG) is a very popular laser host materials, because of its high hardness, high thermal conductivity and stable chemical property. Its cubic crystal structure is also important to achieve high optical transparency. However, processing and fabrication of transparent ceramics with high optical transparency is still a challenge.

The work in this thesis is focused on the development of ytterbium doped transparent laser ceramics, mainly based on YAG garnet. By using high purity $\mathrm{Yb}_{2} \mathrm{O}_{3}, \mathrm{Al}_{2} \mathrm{O}_{3}$ and $\mathrm{Y}_{2} \mathrm{O}_{3}$ powders as starting materials, ytterbium doped YAG (Yb:YAG) ceramics with different concentrations of $\mathrm{Yb}$ were fabricated by using the conventional solid-state reaction process, combined with vacuum sintering technique. XRD results showed that all samples obtained were of pure garnet phase. SEM characterization results revealed that all samples had very dense and pore-free microstructure, with the average grain size of about $10 \mu \mathrm{m}$. 
According to spectroscopic studies, the Yb:YAG laser ceramics exhibited the in-line transmittance of $83 \%$ at room temperature, which was very close to their theoretical transparency. The high optical quality assured that the samples can be used for practical laser applications. As a result, the Yb:YAG ceramics demonstrated absorption and emission cross-section of $0.72 \times 10^{-20} \mathrm{~cm}^{2}$ and $2.01 \times 10^{-20} \mathrm{~cm}^{2}$, respectively. A diode pumped solid state laser system has been set up by using the Yb:YAG ceramics as the laser medium. Continuous wave (CW) laser operation was successfully achieved. The 5.0 at.\% doped Yb:YAG sample had a maximum output power of $6.2 \mathrm{~W}$, corresponding to a laser efficiency of $62 \%$.

Broader gain spectra have been achieved by adjusting the composition of the garnet host materials. In this respect, transparent ytterbium doped gadolinium yttrium aluminum garnets (Yb:GdYAG), i.e., mixed garnet ceramics, have been developed. This new type of mixed garnet ceramics could be used for laser applications with promising performances. Correspondingly, $\mathrm{CW}$ and passive mode-locking laser operations have been experimentally investigated.

The technique developed for $\mathrm{Yb}$ :YAG was further extended to lutetium aluminum garnet (LuAG). This is because LuAG higher thermal conductivity and thus has high potential as the host laser ceramics for high power laser applications, due to the better thermal management. Experimentally, the Yb:LuAG ceramics exhibited an emission crosssection of $2.7 \times 10^{-20} \mathrm{~cm}^{2}$, which was higher than that of $\mathrm{Yb}$ :YAG by about $35 \%$. CW laser performance of the sample was characterized, with an output power of $7.2 \mathrm{~W}$ and slope efficiency of $65 \%$. Femtosecond mode-locked laser operation was also realized with 650 fs pulse duration by using the $\mathrm{Yb}: \mathrm{LuAG}$ ceramics.

Based on the achievements, it is expected that $\mathrm{Yb}$ doped sesquioxide ceramics with even higher thermal properties could be considered to be new laser materials as the future works, which have been supported by preliminary results. 


\section{Acknowledgements}

First and foremost, I would like to give my sincere thanks and appreciation to my supervisor Assoc. Prof. Kong Ling Bing and co-supervisor Assoc. Prof. Tang Dingyuan for offering me the opportunity to take the journey of my $\mathrm{PhD}$ study. Their patient guidance and knowledgeable advice have made this challenging journey into an inspired and fruitful destination.

I would also like to express the acknowledgement to our group members: Dr Zhang Jian, Dr Ning Kaijie, Dr. Ma Jie, Mr Wang Jun and Ms Yin Danlei. It has been a pleasure to work with all of you, as colleagues in work and friends in life. The friendship will always be cherished.

Thanks will also be given to the technicians and staffs in the MSE lab who have helped a lot in one way or another.

Last but not least, my gratitude will be given to my parents. Their love and encouragement will always be the strongest support in my life!

Once again, thank all of you! 


\section{Table of Contents}

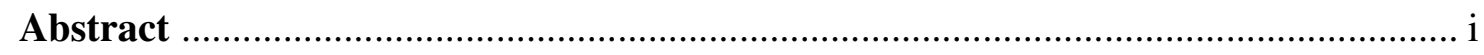

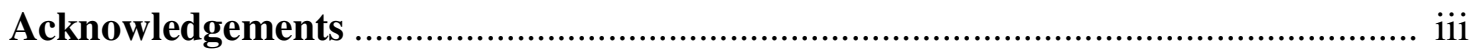

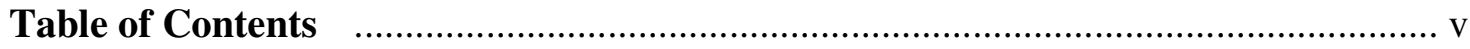

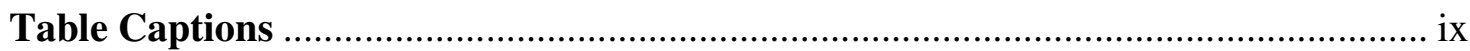

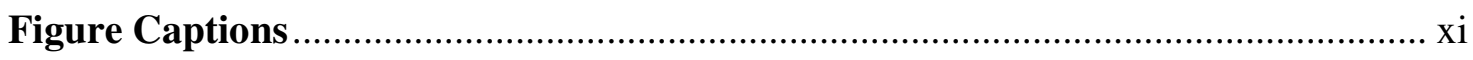

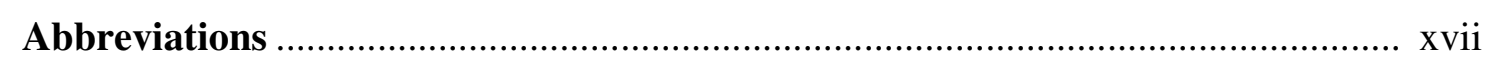

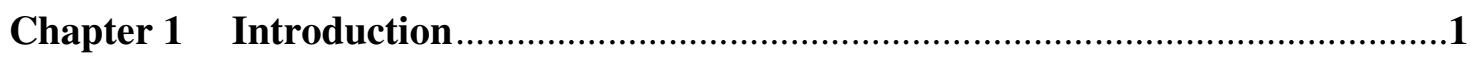

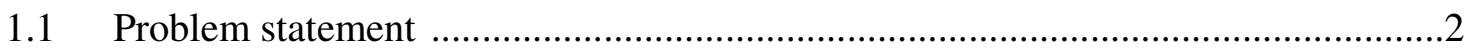

1.2 Objectives and scope

1.2.1 Establish fabrication method for laser ceramics .........................................

1.2.2 Develop new laser ceramic materials ......................................................4

1.2.3 Demonstration of the ceramic laser performances .....................................

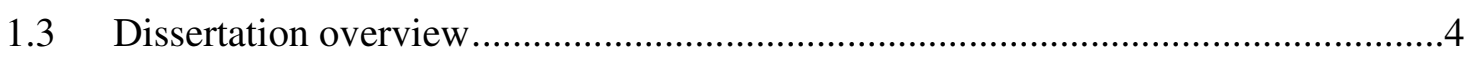

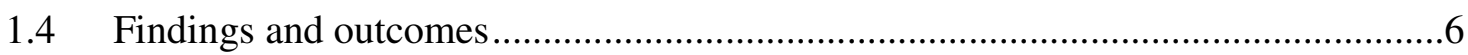

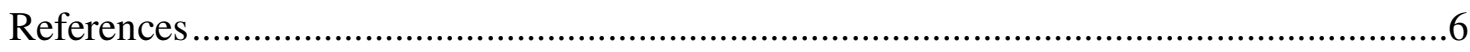

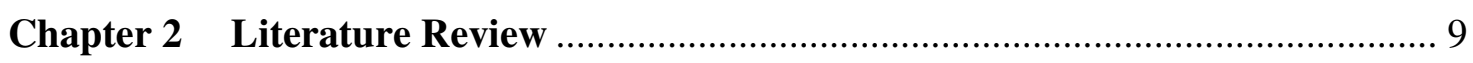

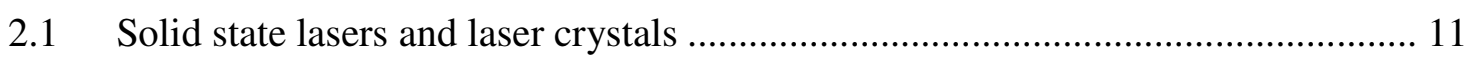

2.2 Polycrystalline transparent ceramics as laser materials ...................................... 12

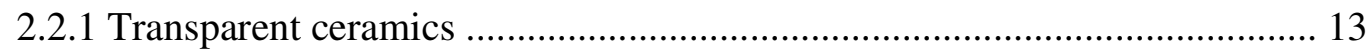




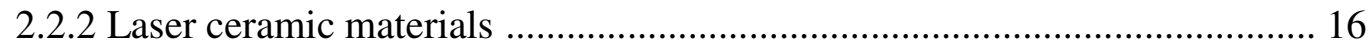

2.3 Fabrication approach for laser ceramics............................................................ 19

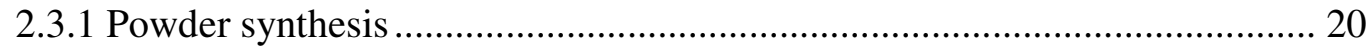

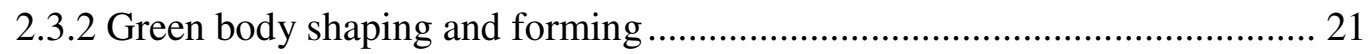

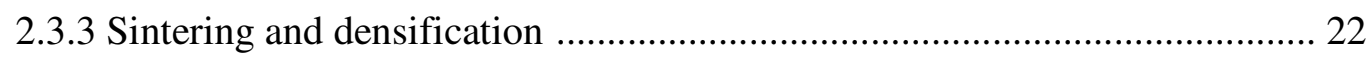

2.3.3.1 Sintering mechanisms _............................................................. 24

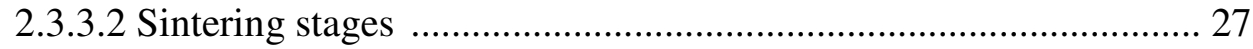

2.4 Challenges in transparent laser ceramics …...................................................... 31

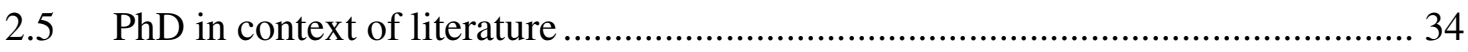

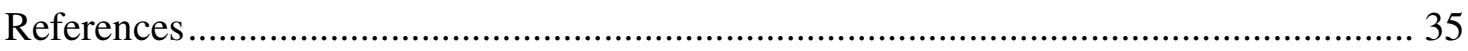

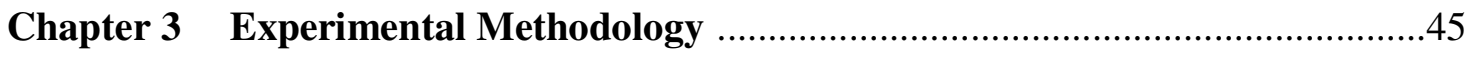

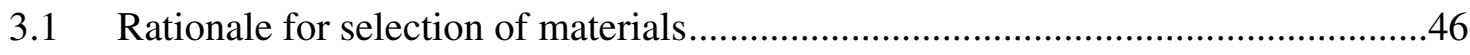

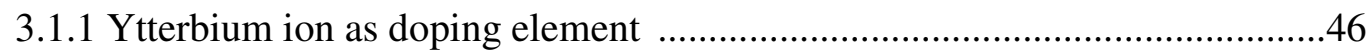

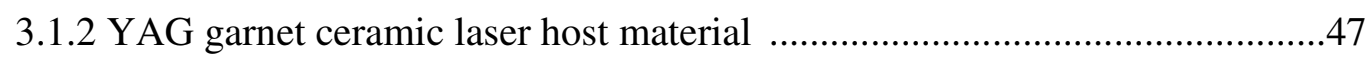

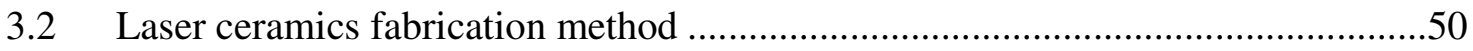

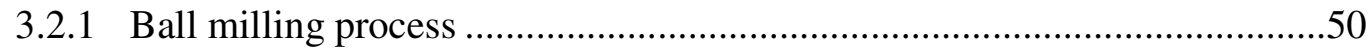

3.2.2 Dry press and cold isostatic pressing process ..........................................51

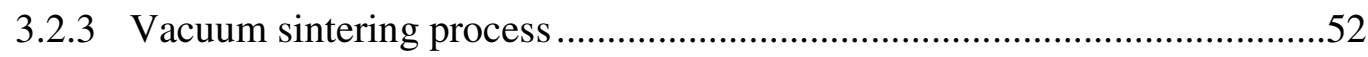

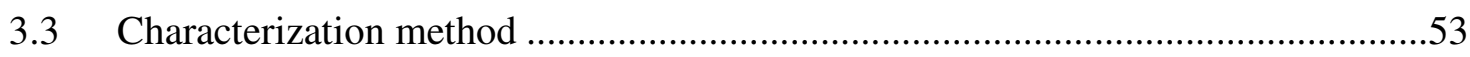

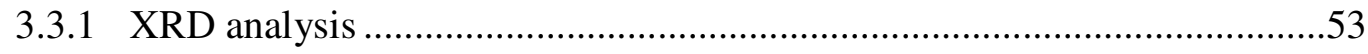

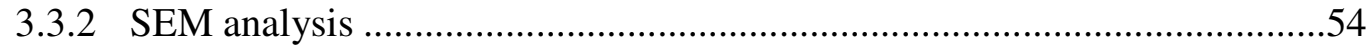

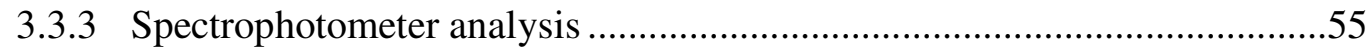

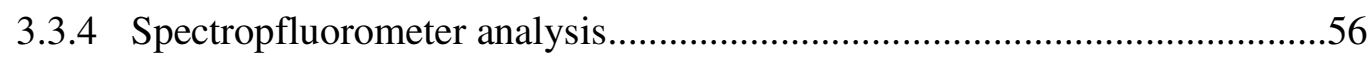

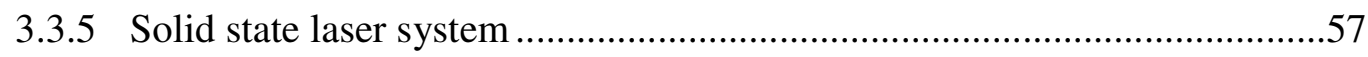




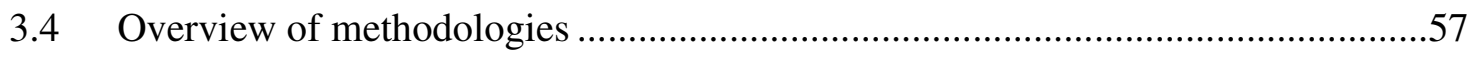

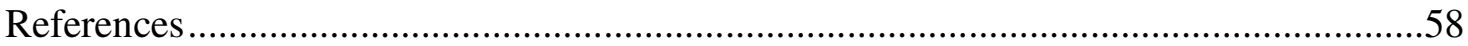

Chapter 4 Development of Yb:YAG Transparent Laser Ceramics .......................61

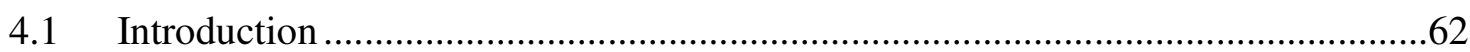

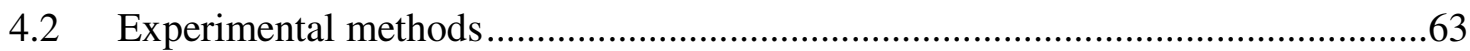

4.2.1 Fabrication process of Yb:YAG laser ceramics.......................................63

4.2.2 Characterization of Yb:YAG laser ceramics .........................................65

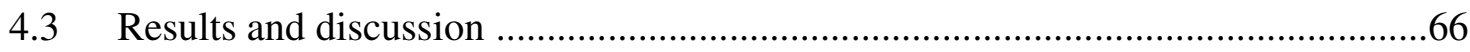

4.3.1 Phase and microstructure study ..........................................................66

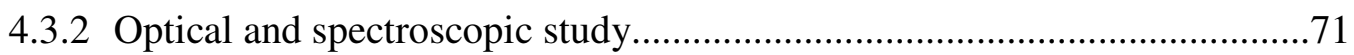

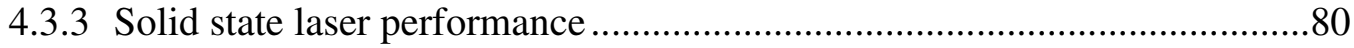

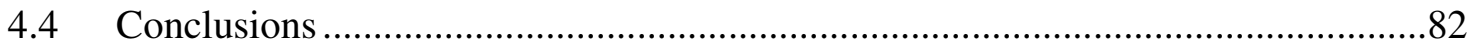

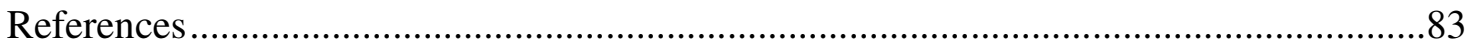

Chapter 5 Development of Yb:GdYAG Mixed Garnet Laser Ceramics ................87

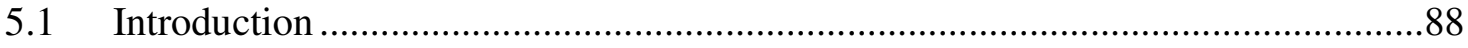

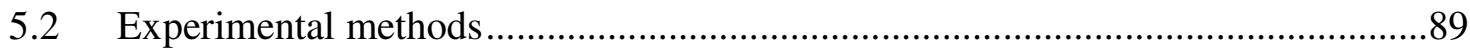

5.2.1 Fabrication and characterization of Yb:GdYAG laser ceramics .................89

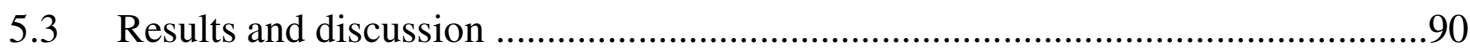

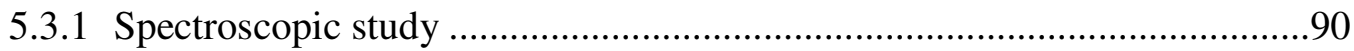

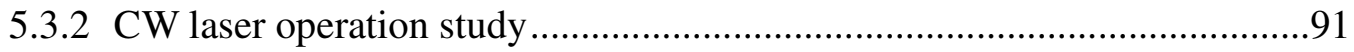

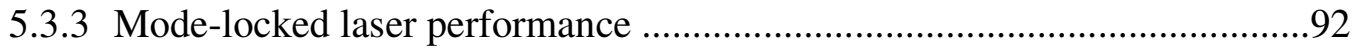

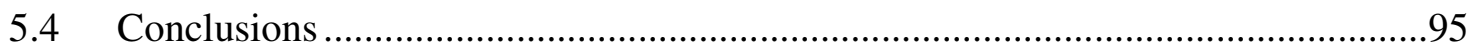

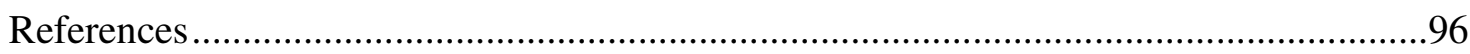


Chapter 6 Development of Yb:YLuAG Laser Ceramics.........................................99

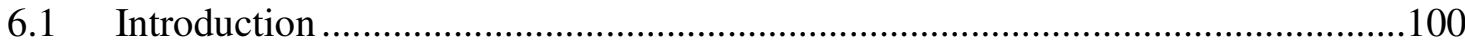

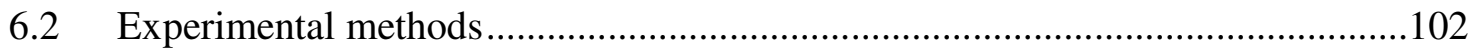

6.2.1 Fabrication of Yb:YLuAG laser ceramics ............................................102

6.2.2 Characterization of $\mathrm{Yb}: \mathrm{YLuAG}$ laser ceramics .......................................104

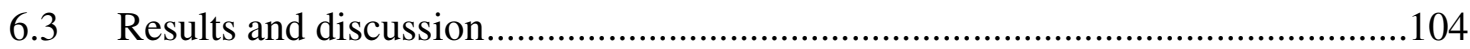

6.3.1 Phase and microstructure study .........................................................104

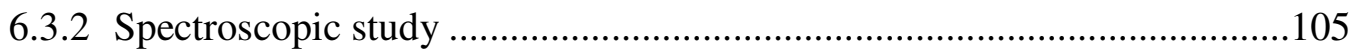

6.3.3 CW and mode-locked laser performance................................................108

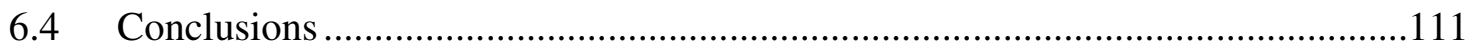

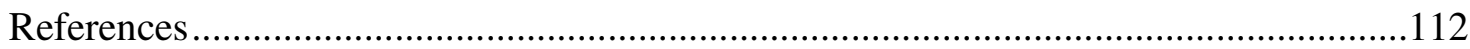

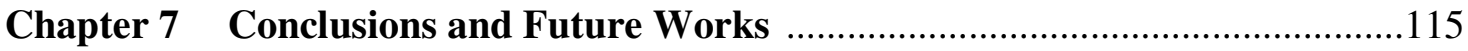

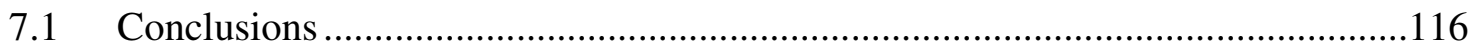

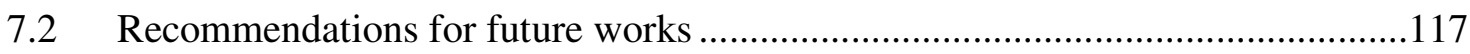

7.2.1 Exploration for new type of ytterbium co-doped garnet laser ceramics ....117

7.2.2 Composite structures laser ceramics ....................................................118

7.2.3 Sesquioxide ceramics as laser host material ...........................................119

7.1.4 Residual pore removal and ceramics densification study ........................120

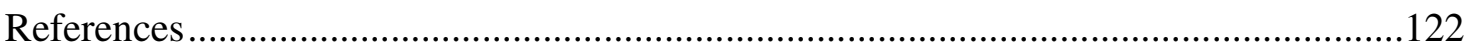

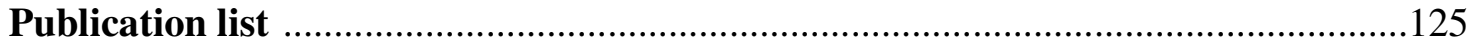




\section{Table Captions}

Table 2.1 Ceramic green body shaping method.

Table 2.2 Material transport mechanisms of sintering.

Table 3.1 Properties of YAG crystal.

Table 4.1 Composition parameters of Yb:YAG ceramics with different doping concentration.

Table 7.1 Properties of sesquioxides compared with YAG. 


\section{Figure Captions}

Figure 2.1 Schematic drawing of $\mathrm{Cz}$ furnace (left) and a grown single crystal ingot (right).

Figure 2.2 Photos of traditional ceramics (left) and industrial ceramics (right).

Figure 2.3 Schematic drawing of light scattering factors in polycrystalline ceramics.

Figure 2.4 Photo of applications in transparent ceramics.

Figure 2.5 Development progresses of ceramics lasers.

Figure 2.6 General fabrication patterns of laser ceramics.

Figure 2.7 Ceramics sintering process.

Figure 2.8 Illustrations of different types of sintering technologies.

Figure 2.9 Reduction in total interfacial energy through densification and grain coarsening during sintering process.

Figure 2.10 Six mechanisms contributing to the sintering of crystalline ceramics.

Figure 2.11 Idealized models for the three stages of sintering: (a) Initial stage, (b) Towards the end of initial stage, (c) Intermediate stage and (d) Final stage.

Figure 2.12 Schematic drawing of the densification curve for a powder compact and the three sintering stages.

Figure 2.13 Fabrication process for Nd:YAG ceramics by solid-state reactive sintering. 
Figure 2.14 Fabrication process for Nd:YAG ceramics by a wet chemical method.

Figure 3.1 Energy levels of $\mathrm{Yb}^{3+}$ ions.

Figure 3.2 Phase diagram of $\mathrm{Al}_{2} \mathrm{O}_{3}-\mathrm{Y}_{2} \mathrm{O}_{3}$ composite system.

Figure 3.3 Crystal structure of YAG.

Figure 3.4 Schematic drawing of high energy planetary ball milling system with (left) layout of planetary disk and (right) horizontal view of milling jar.

Figure 3.5 Schematic diagrams of the uniaxial mold and cold isostatic pressing.

Figure 3.6 Photo of high temperature vacuum sintering furnace.

Figure 3.7 Schematic drawing of Bragg diffraction.

Figure 3.8 Schematic drawing of SEM equipment.

Figure 3.9 Schematic drawing of configuration of a spectrophotometer.

Figure 3.10 Schematic drawing of configuration of a spectrofluorometer.

Figure 3.11 Schematic drawing of a typical solid state laser system.

Figure 4.1 Fabrication flow chart of Yb:YAG laser ceramics.

Figure 4.2 Photos of (a) green bodies and (b) sintered samples of the Yb:YAG ceramic.

Figure 4.3 SEM images of raw powders: (a) $\mathrm{Al}_{2} \mathrm{O}_{3}$, (b) $\mathrm{Y}_{2} \mathrm{O}_{3}$, (c) $\mathrm{Yb}_{2} \mathrm{O}_{3}$ and (d) mixture after ball milling. 
Figure 4.4 Relative density and grain size of 5.0 at.\% Yb:YAG ceramics.

Figure 4.5 Surface SEM images of 5.0 at.\% Yb:YAG ceramics samples sintered at (a) $1400^{\circ} \mathrm{C}$, (b) $1500^{\circ} \mathrm{C}$, (c) $1550^{\circ} \mathrm{C}$, (d) $1600^{\circ} \mathrm{C}$, (e) $1650^{\circ} \mathrm{C}$ and (f) $1700^{\circ} \mathrm{C}$.

Figure 4.6 XRD patterns of $\mathrm{Yb}$ :YAG ceramics of different $\mathrm{Yb}$ doping concentration.

Figure 4.7 Thermal etched SEM images of the Yb:YAG ceramics: (A) 1 at.\%, (B) 8 at.\%, (C) 15 at.\% and (D) 20 at.\% doping concentration.

Figure 4.8 Fracture SEM images of the Yb:YAG ceramics: (A) 1 at.\%, (B) 8 at.\%, (C) 15 at.\% and (D) 20 at.\% doping concentration.

Figure 4.9 SEM images of the 5.0 at.\% Yb:YAG ceramics sintered for: (a) $8 \mathrm{~h}$, (b) $12 \mathrm{~h}$, (c) $24 \mathrm{~h}$ and (d) $30 \mathrm{~h}$.

Figure 4.10 Transmittance curves of the 1.0 at.\% Yb:YAG ceramics before and after annealing, with their photos shown in the inset.

Figure 4.11 Transmittance curves of the 5.0 at.\% Yb:YAG ceramics with different sintering time.

Figure 4.12 Transmittance curves of the Yb:YAG ceramic samples (annealed) with various doping concentration.

Figure 4.13 Absorption spectra of the Yb:YAG ceramics with different $\mathrm{Yb}$ doping concentrations.

Figure 4.14 Absorption coefficient of Yb:YAG ceramics at $940 \mathrm{~nm}$ with respective to the doping concentration. 
Figure 4.15 The (a) fluorescence spectrum and (b) fluorescence decay curve of the 5.0 at.\% Yb:YAG ceramics.

Figure 4.16 Absorption and emission cross-section of the Yb:YAG ceramics.

Figure 4.17 Gain cross-section of the Yb:YAG ceramics under different $\beta$.

Figure 4.18 The cavity configuration of a CW Yb:YAG ceramics laser.

Figure 4.19 Laser performance of the Yb:YAG ceramics.

Figure 5.1 The room temperature absorption and normalized emission spectra of a 5.0 at.\% Yb:GdYAG ceramic.

Figure 5.2 The cavity configuration of a Yb:GdYAG ceramic laser in CW operation.

Figure 5.3 Output power versus the incident pump power of a 5.0 at.\% Yb:GdYAG ceramic under $\mathrm{CW}$ operation.

Figure 5.4 The experimental setup for mode-locked Yb:GdYAG ceramic laser.

Figure 5.5 The Output power of mode-locked Yb:GdYAG ceramic laser with respect to the incident pump power.

Figure 5.6 Autocorrelation trace of the mode-locked Yb:GdYAG ceramics laser.

Figure 5.7 Optical spectrum of mode-locked pulse for Yb:GdYAG ceramics laser.

Figure 6.1 $\mathrm{Lu}_{2} \mathrm{O}_{3}-\mathrm{Al}_{2} \mathrm{O}_{3}$ phase diagram.

Figure 6.2 Thermal conductivity of $\mathrm{Yb}$ :YAG and $\mathrm{Yb}: \mathrm{LuAG}$ as a function of $\mathrm{Yb}$ doping 
concentration.

Figure 6.3 In-house fabricated Yb:YLuAG ceramics.

Figure 6.4 $\mathrm{Yb}$ :LuAG ceramic samples sintered at $1840^{\circ} \mathrm{C}$ for $8 \mathrm{~h}$ with different doping concentration.

Figure 6.5 XRD patterns of YLuAG ceramics.

Figure 6.6 Surface SEM images of the $\mathrm{Yb}: \mathrm{LuAG}$ ceramics sintered at $1840^{\circ} \mathrm{C}$ for $8 \mathrm{~h}$ with different doping concentrations: (a) 5 at.\%, (b) 10 at.\%, (c) 15 at.\% and (d) 20 at.\%.

Figure 6.7 Absorption spectra of $\mathrm{Yb}$ :LuAG ceramic samples with different $\mathrm{Yb}$ doping concentrations.

Figure 6.8 Emission cross-section and decay curve (inset) of the 5.0 at.\% Yb:LuAG ceramics.

Figure 6.9 Gain cross-section of $\mathrm{Yb}: \mathrm{LuAG}$ ceramics under different value of inversion ratio $\beta$.

Figure 6.10 $\mathrm{CW}$ laser performance of the 5.0 at.\% Yb:LuAG ceramics under different output couplers.

Figure 6.11 Schematic drawing of cavity design of passively mode-locked Yb:LuAG ceramic laser.

Figure 6.12 Autocorrelation trace of the mode-locked Yb:LuAG ceramics laser with laser spectrum shown as the inset.

Figure 7.1 Transmittance curve of $\mathrm{Er}, \mathrm{Yb}$ co-doped YAG ceramics with photo of 
samples in the insert.

Figure 7.2 Photograph of composite $\mathrm{Yb}$ :YAG laser ceramics.

Figure 7.3 Model for the pore elimination of hot isostatic pressing sintering. 


\section{Abbreviations}

$\begin{array}{ll}\text { CIP } & \text { Cold Isostatic Pressing } \\ \mathrm{CW} & \text { Continuous Wave } \\ \mathrm{Cz} & \text { Czochealski } \\ \text { LuAG } & \text { Lutetium Aluminum Garnet } \\ \text { SEM } & \text { Scanning Electron Microscopy } \\ \text { SSL } & \text { Solid State Laser } \\ \text { XRD } & \text { X-ray Diffraction } \\ \text { YAG } & \text { Yttrium Aluminum Garnet } \\ \text { Z } & \text { Atomic Number }\end{array}$




\section{Chapter 1}

\section{Introduction}

Solid state lasers have shown enormous applications in military, industry and medical fields. Great efforts have been made to develop high quality laser materials to fulfill the requirements of these applications. With the advancement of modern ceramic processing technology, polycrystalline ceramic materials have shown great potentials as the laser gain medium, compared with the traditional single crystal laser materials. However, developing new types of laser ceramic materials and improving their qualities are still challenges to be faced by the research community of this area. This chapter was aimed to provide a brief introduction of laser ceramic materials, followed by an outline on the structure of the whole thesis. Also, objectives and scope of the thesis are described, while outcomes and findings are briefly summarized. 


\subsection{Problem statement}

Solid state lasers (SSL) have shown enormous applications in scientific study, military, industry and medical fields [1-4]. Since the first ruby laser was invented by Maiman in the 1960s [5,6], various type of SSLs have been developed. Such as continuous wave (CW) Nd:YAG single crystal laser [7], Ti:sapphire single crystal pulse laser [8]. As the active medium in the laser system, laser crystals often play the role of the most important component in the SSL system. The quality of laser crystals directly affects the whole laser system's performance and efficiency. Numerous types of materials have also been tried and tested as laser crystals for different application usage. Crystalline material such as garnet materials (YAG, GGG, GSGG) [7, 9, 10], fluoride materials $\left(\mathrm{CaF}_{2}, \mathrm{MgF}_{2}\right)$ [11, 12], vanadate materials [13], as well as amorphous materials such as silicate glass [14] have been successfully developed. The laser materials must have these basic requirements such as excellent optical quality with high transparency, strong mechanical properties of high hardness, stable chemical properties to resist chemical corrosion, and good thermal properties such as high thermal conductivity and low thermal expansion. Great efforts have also been put to develop good quality laser materials to fulfill these application usages [15-17].

Traditional single crystals such as yttrium aluminum garnet (YAG) crystals are usually grown by the Czochealski $(\mathrm{Cz})$ method [18]. This technique often takes quite a long production period and involves very expensive equipment and facilities. The directly obtained crystal ingots also encounter the problems such as non-homogeneity of materials doping concentration and optical quality. The final portion which is suitable to be used in practical laser system is only limited. So it means a relatively low yield of production, which in turns as high unit cost. Therefore, either improvement in the crystal grow technology or finding another alternate way of obtaining high quality and high productivity laser materials have become the problems waiting to be solved.

With the advancement in modern ceramics processing technology, polycrystalline transparent ceramic materials have shown great potentials as the laser material comparing 
with the traditional single crystal laser material [19-24]. A ceramic is an inorganic, nonmetallic solid material. It possesses the similar mechanical, chemical and thermal properties as single crystal, but only in the form of polycrystalline. The main processing steps for ceramics usually consist the followings: (1) synthesis and preparation of the raw powder materials, (2) compaction of the powders to form the green bodies, and (3) sintering and densification. The fabrication nature is very different from single crystal growth. Each processing step has a great influence on the property and optical quality of the final ceramic products. There are various processing parameters and different methods of sintering techniques to be utilized to optimize the ceramics fabrication process. As early as in the 1950 s, Coble et al. from MIT has fabricated translucent alumina ceramics by controlling its microstructure [25], thus opening the era of ceramics in the optical application. In 1964 the first laser oscillation was realized by using the Dy: $\mathrm{CaF}_{2}$ ceramics as the laser gain material [12]. In 1973 the first pulse laser oscillation of $\mathrm{Nd}: \mathrm{ThO}_{2}-\mathrm{Y}_{2} \mathrm{O}_{3}$ ceramics was reported [26]. However the laser efficiency achieved at that time was extremely low.

It was until in 1995 when the breakthrough was made by Ikesue et al. by using an improved ceramics fabrication technology to obtain the Nd:YAG ceramics of optical quality good enough to achieve efficient laser oscillation [27]. This proves the possibility of using polycrystalline ceramics as laser material. Since then, more and more ceramic lasers have been reported [29-31]. Researchers are still continuously putting efforts in improving the quality of laser ceramics, as well as developing new types of laser ceramic materials to fulfill the requirements for different laser applications. Nowadays, the highest output power of ceramics lasers can reach the value of more than $100 \mathrm{~kW}$ [32]. In addition, the ceramics technology can break the limitation of $\mathrm{Cz}$ growth method. For example, ceramics samples can be fabricated with theoretically unlimited size and complex structure design. Yet the fabrication for good quality laser ceramics still remains a challenging task. This thesis will focus on the establishment of fabrication approaches and optimization on improving the optical quality transparent laser ceramics, as well as the development of new types of laser materials. Laser performances testing of obtained samples will also be carried out. 


\subsection{Objectives and scope}

\subsubsection{Establish fabrication method for laser ceramics}

Though there's number of reports focused on fabrication approach for good quality laser ceramics, optimization and improvement for the optical quality of laser ceramics are still possible. We will demonstrate that by using combination of advanced ceramic processing technology, laser ceramics with high optical quality will be obtained. The optimized fabrication method for laser ceramics will be established.

\subsubsection{Develop new laser ceramic materials}

The laser ceramics processing technology is not limited to only one specific type of laser ceramic material. Based on the established fabrication method, new laser ceramics material of possibility will also be explored. It is possible that the fabrication method can be applied on various kinds of new materials with slight modification of fabrication conditions and parameters.

\subsubsection{Demonstration of ceramic laser performances}

Solid state laser system will be set up using the in-house fabricated ceramics as laser gain medium. The laser performance is also a good reflection of the quality of ceramics obtained. Good optical quality of the fabricated ceramics will lead to high power lasers with high slope efficiency.

\subsection{Dissertation Overview}

Outline of the thesis is described as follows:

Chapter 1 gives the problem statement and provides a rationale for the research. The objective and scope as well as findings and outcomes are outlined. 
Chapter 2 reviews the background knowledge of solid state laser and laser materials. The historical development progress and current situation in the area of transparent laser ceramics are also reviewed. The fabrication technologies of transparent laser ceramics are introduced. Challenging and difficulties in obtaining high quality laser ceramics are discussed.

Chapter 3 discusses the experimental methods and rationale for obtaining high quality ytterbium doped laser ceramics. Ceramic fabrication technologies such has ball milling process, green-body shaping process and vacuum sintering techniques are introduced. SEM and XRD are used to characterize the phase and microstructure of the ceramic samples. Spectrophotometer and spectrofluorometer are used to measure sample's spectroscopic properties. Solid state laser systems are also introduced to test the samples' laser performance.

Chapter 4 presents the development for fabricating high quality ytterbium (Yb) doped YAG laser ceramics. High purity commercial powders are used as starting material. High energy ball milling technique is used for powder processing. Dry press and cold isostatic pressing (CIP) techniques are used for green-body forming. Vacuum sintering is used for ceramic samples densification. Phase and microstructure morphology of the samples are characterized. Sintering densification progress is studied. Optical and spectroscopic properties of samples are investigated. And the laser performances of obtained samples are demonstrated.

Chapter 5 presents the fabrication and characterization for obtaining a new type of $\mathrm{Yb}$ doped GdYAG mixed garnet laser ceramics by using the vacuum sintering method demonstrated in the previous chapter. Characterizations of in-house fabricated Yb:GdYAG ceramics are also carried out. CW and mode-locked laser operations of the samples are successfully demonstrated.

Chapter 6 presents the development of the YLuAG garnet ceramics as an alternate host material for the $\mathrm{Yb}$ ion. The new type of $\mathrm{Yb}: \mathrm{LuAG}$ ceramics have been obtained 
successfully by vacuum sintering method, which was firstly reported. Characterizations Yb:YLuAG ceramics were also carried out. $\mathrm{CW}$ and mode-locked laser system are built and femtosecond ultrafast laser operations are successfully realized.

Chapter 7 concludes and summaries the techniques in obtaining high quality $\mathrm{Yb}$ doped garnet laser ceramics. Although good results were presented in the previous chapters, there's still space for further improvement of the sample quality as well as the exploration for new laser ceramic materials. Suggestions and recommendation for future work and some preliminary results in laser ceramics are also discussed.

\subsection{Findings and outcomes}

The thesis work led to the following novel outcomes:

(1) A fabrication technique to achieve high performance $\mathrm{Yb}$ doped YAG garnet laser ceramics has developed. Successful laser operations with high efficiency have been achieved by using the Yb:YAG ceramics as the laser gain medium.

(2) New type of $\mathrm{Yb}$ doped GdYAG mixed garnet ceramics and $\mathrm{Yb}$ doped LuAG garnet ceramics have been fabricated by using the developed technique, which have been reported for the first time.

(3) $\mathrm{CW}$ and mode-locked laser operations by using the transparent ceramics as laser gain medium have been realized. Laser performances of the Yb-doped ceramics with high slope efficiency have been demonstrated.

\section{References}

[1] W. Koechner, and M. Bass, Solid-State Lasers: A Graduate Text. Springer Science \& Business Media, 2003.

[2] T. O. Hardwell, Solid-state Lasers: Properties and Applications. Nova Publishers, 2008.

[3] W. Koechner, Solid-state laser engineering. Springer, 2013. 
[4] F. Hide, M. A. Diaz-Garcia, B. J. Schwartz and M. R. Andersson, Science. 1996, $273,1833$.

[5] T. H. Maiman, Nature. 1960, 187, 493-494.

[6] T. Maiman, Physical Review Letters. 1960, 4, 564.

[7] J. Geusic, H. Marcos, and L. Van Uitert, Applied Physics Letters. 1964, 4, 182-184.

[8] P. Moulton, Optics News. 1982, 8, 9-9.

[9] Q. Xiao, and J. J. Derby, Journal of Crystal Growth. 1994, 139, 147-157.

[10] W. Krupke, M. Shinn, J. Marion, J. Caird, and S. Stokowski, Journal of the Optical Society of America B. 1986, 3, 102-114.

[11] D. Welford, and P. F. Moulton, Optics letters. 1988, 13, 975-977.

[12] S. Hatch, W. Parsons, and R. Weagley, Applied Physics Letters. 1964, 5, 153-154.

[13] J. Rubin, and L. Van Uitert, Journal of Applied Physics. 1966, 37, 2920-2921.

[14] M. Ledig, E. Heumann, D. Ehrt, and W. Seeber, Optical and quantum electronics. 1990, 22, S107-S122.

[15] R. BYER, Science. 1988, 239, 742-747.

[16] S. A. Payne, L. Chase, H. W. Newkirk, L. K. Smith, and W. F. Krupke, IEEE Journal of Quantum Electronics. 1988, 24, 2243-2252.

[17] A. Giesen, H. Hügel, A. Voss, K. Wittig, U. Brauch, and H. Opower, Applied Physics B. 1994, 58, 365-372.

[18] R. C. Linares, Solid State Communications. 1964, 2, 229-231.

[19] A. Ikesue, I. Furusato, and K. Kamata, Journal of the American Ceramic Society. 1995, 78, 225-228.

[20] A. Ikesue, Y. L. Aung, T. Taira, T. Kamimura, K. Yoshida, and G. L. Messing, Annual Review of Materials Research. 2006, 36, 397-429.

[21] A. Ikesue, and Y. L. Aung, Nature photonics. 2008, 2, 721-727.

[22] J. Sanghera, W. Kim, G. Villalobos, B. Shaw, C. Baker, J. Frantz, B. Sadowski, and I. Aggarwal, Materials. 2012, 5, 258-277.

[23] V. Lupei, A. Lupei, and A. Ikesue, Optical Materials. 2008, 30, 1781-1786.

[24] J. Sanghera, W. Kim, G. Villalobos, B. Shaw, C. Baker, J. Frantz, B. Sadowski, and I. Aggarwal, Optical Materials. 2013, 35, 693-699.

[25] R. Coble, American Ceramic Society Bulletin. 1959, 38, 507-510. 
[26] C. Greskovich, and J. Chernoch, Journal of Applied Physics. 1973, 44, 4599-4606.

[27] A. Ikesue, T. Kinoshita, K. Kamata, and K. Yoshida, Journal of the American Ceramic Society. 1995, 78, 1033-1040.

[28] X. Qin, H. Yang, G. Zhou, D. Luo, Y. Yang, J. Zhang, S. Wang, J. Ma, and D. Tang, Optical Materials. 2012, 34, 973-976.

[29] H. Yang, J. Zhang, X. Qin, D. Luo, J. Ma, D. Tang, H. Chen, D. Shen, and Q. Zhang, Journal of the American Ceramic Society. 2012, 95, 52-55.

[30] Y. Wang, D. Shen, H. Chen, J. Zhang, X. Qin, D. Tang, X. Yang, and T. Zhao, Optics Letters. 2011, 36, 4485-4487.

[31] J. Zhang, L. An, M. Liu, S. Shimai, and S. Wang, Journal of the European Ceramic Society. 2009, 29, 305-309.

[32] A. Mandl, and D. E. Klimek, presented at Conference on Lasers and Electro-Optics. 2010, JThH2. 


\section{Chapter 2}

\section{Literature Review}

Transparent laser ceramics have formed a new group of functional materials that can be used in solid state laser systems. In this chapter, the background knowledge of solid state laser and laser crystal materials are introduced. The historical development progress and the current situation in the area of transparent ceramics as laser materials are presented and discussed. The fabrication technologies for transparent ceramics are described in a more detailed way. Challenging and difficulties in developing high quality laser ceramics are discussed. 


\subsection{Solid state lasers and laser crystals}

The word LASER is an acronym stands for light amplification by stimulated emission of radiation [1-4]. The first laser device was a pulsed ruby laser, demonstrated by Theodore Maiman in the 1960s [5, 6]. Since then lasers have played an important role in science, military, medical and industry applications [7-13]. A complete laser system has three main components: energy source, laser cavity and laser gain medium [1]. The laser gain medium is the source of optical gain within a laser system. The gain results from the stimulated emission of electronic or molecular transitions to a lower energy state from a higher energy state previously populated by a pump source. The laser cavity is where the energy source interacts with the gain medium and creates amplification. Lasers can be generally categorized into four kinds depending on the difference of laser gain medium: (1) semiconductor lasers, (2) gas lasers, (3) dye lasers, and (4) solid state lasers (SSL). Although semiconductor lasers are made of solid materials, they are still considered an independent laser type. They are mainly used for optical communications and information storage [14-16]. He-Ne gas lasers are typically used for distance measurement $[17,18]$ and industrial often use $\mathrm{CO}_{2}$ gas lasers as the cutting and welding tools $[19,20]$. Dye lasers use organic dye as laser medium [21, 22]. Solid state lasers use ion doped bulk solids such as glass or crystalline materials as laser medium [1, 23-25]. Typical solid state lasers can generate high output powers with high beam quality or ultra-short pulses for mechanical or medical applications.

Various solid materials have been developed for solid state lasers as the laser gain medium. Crystalline laser materials include garnets, such as yttrium aluminum garnet (YAG) [26-29], gadolinium gallium garnet (GGG) [30, 31] and gadolinium scandium gallium garnet (GSGG) [32, 33], fluoride materials include $\mathrm{CaF}_{2}$ and $\mathrm{MgF}_{2}$ [34-36], vanadates such as $\mathrm{GdVO}_{4}$ and $\mathrm{YVO}_{4}$ [37-40], alexandrite [41, 42] and forsterite [43, 44]. Non-crystalline materials are usually amorphous silicate and phosphate glasses $[45,46]$. These solid laser materials must meet the basic requirement for practical laser applications: (1) Excellent optical quality, which means less scattering and high optical transmittance. (2) Good mechanical property, include high hardness and fracture 
toughness. (3) Stable chemical property to resist chemical corrosion and reaction. (4) High thermal conductivity and low thermal expansion coefficient in order to withstand thermal short in high power laser operations. (5) Suitable spectroscopic property for different laser application purposes.

Traditional laser crystals are usually grown by using the Czochralski $(\mathrm{Cz})$ method. It is named after a scientist Jan Czochralski as early as 1916 [47] when he was experimenting on the crystallization rate of metals. Later this method was modified by Teal et al. to become known as the Czochralski method [48]. It is still a very popular crystal growth method utilized by many researchers to grow various laser crystals up till now [49-52].
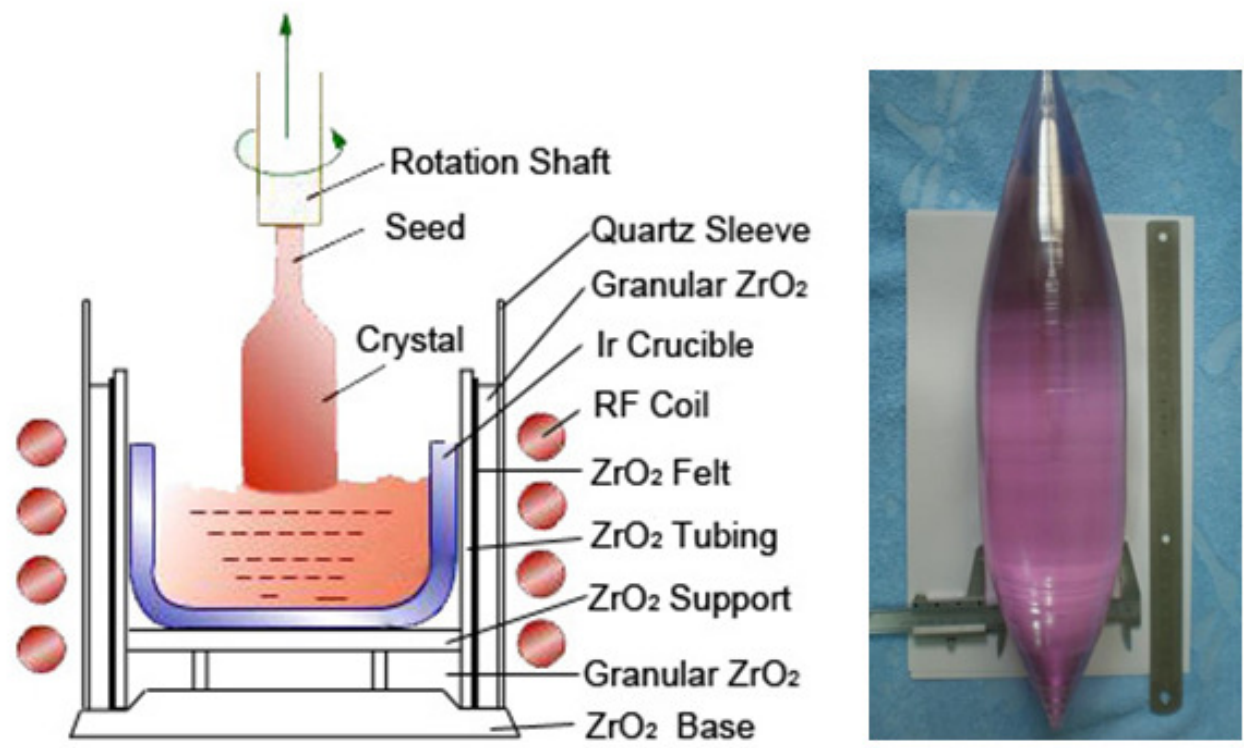

Figure 2.1 Schematic drawing of $\mathrm{Cz}$ furnace (left) and a grown single crystal ingot (right).

The Czochralski growth of crystal is a melt-grown process. The crucible is heated up using induction generators to the temperature above the melting point of the grown crystal. Typically it is close to or above $2000^{\circ} \mathrm{C}$. Very expensive iridium (Ir) crucible of which the highest working temperature is up to $2200^{\circ} \mathrm{C}$ is often used. The raw materials of crystal are blended into desired chemical stoichiometric composition and melted in the 
crucible. A seed crystal is placed on top of the melt and is constantly rotated and slowly pulled up from the melt. Temperature and pulling rate and speed is carefully controlled so that the solid crystal is formed and "grown" from the seed. Figure 2.1 presents the schematic drawing of Czochralski furnace and a grown single crystal ingot [53].

This technique often takes quite a long production period because the growing rate is usually less than $1 \mathrm{~mm}$ per hour. The diameter of crystal gown is also limited by the size of crucible and furnace, usually the ingot diameter is less than 10 inches. This means large size production will need even more expensive equipment and facilities. The directly obtained crystal ingots also encounter crystal detects, such as cores and facets, as well as the problems such as non-homogeneity of materials doping concentration [54]. The final portion of the whole crystal which is suitable to be used in practical laser system is only limited. Only the part with perfect quality will be cut off and further polished and processed. So it means a relatively low yield of production, causing a lot of waste in materials, which in turns as high unit cost. Thus, obtaining relatively large size and high quality laser crystal for future high power lasers still remains a challenging problem by the current crystal growing technology. Therefore, either improvement in the crystal growth technology or finding other alternate ways of obtaining high quality and high productivity laser materials have also become the problems waiting to be solved.

\subsection{Polycrystalline transparent ceramics as laser materials}

Ceramics comes from Greek word "keramos", which means pottery and porcelain. They were invented by humans thousands of years ago in ancient times. A ceramic is an inorganic, nonmetallic solid material [55]. It is often being used as earthenware or tableware in households and is usually made from naturally occurring clay and sand. With the increase in varieties and the advancement of material processing technologies, ceramics are made into a wide range of industrial products showing their great usage in modern era [56]. How ceramics are involved as the laser materials will be discussed in this section. 


\subsubsection{Transparent ceramics}

A ceramic material is an inorganic, nonmetallic material. They are often crystalline oxide, nitride or carbide in chemical composition. Ceramic materials are brittle, hard, and strong in compression, weak in shearing and tension. They have relatively stable physical and chemical properties that can help them resist chemical erosion and physical wear and tear. Ceramics can also generally withstand very high temperatures.

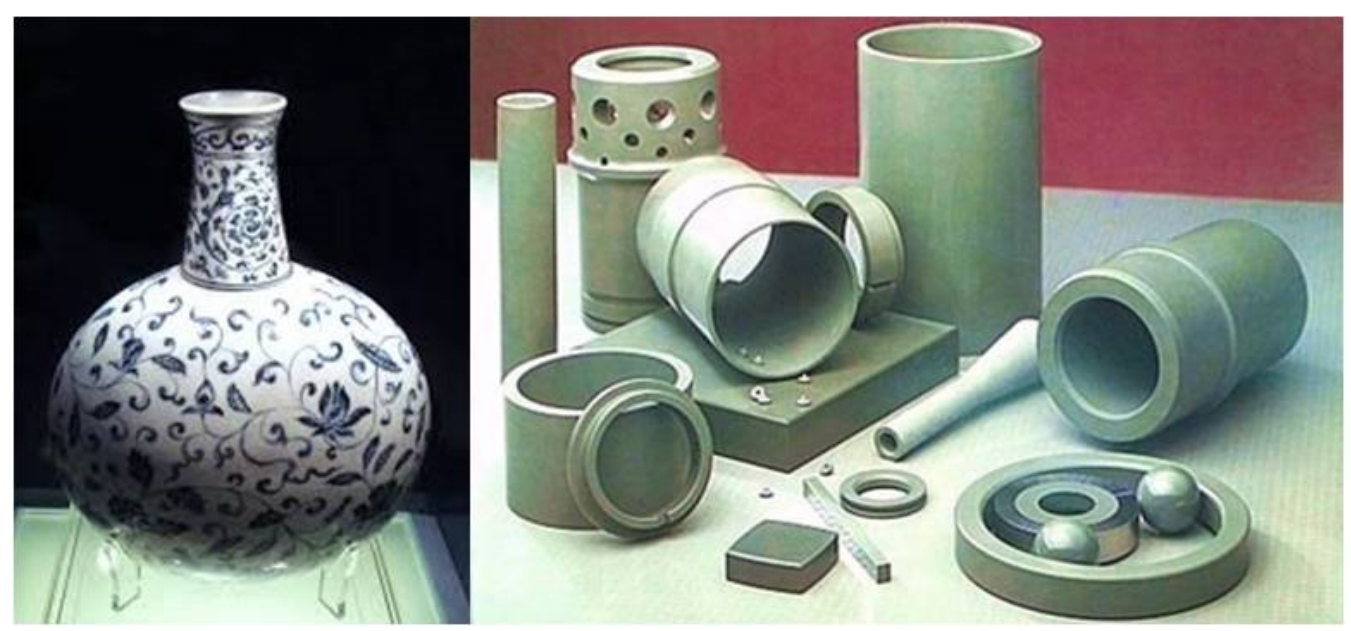

Figure 2.2 Photographs of traditional ceramics (left) and engineering ceramics (right).

Figure 2.2 shows the photo of traditional and industrial ceramics. They often take advantages of its high hardness and good mechanical strength. Such as alumina, zirconia and silicon carbide [57-60]. Others use their electrical properties, such as ferroelectric and piezoelectric properties. Examples are lead zirconate titanate (PZT) ceramics [61, 62]. Different from single crystal, the polycrystalline ceramics are composed of many randomly oriented grains. Most of ceramic products are optically either opaque or translucent. Figure 2.3 shows Schematic drawing of optical scattering factors in polycrystalline ceramics which affect its optical transparency [63]. They are summarized as the following factors:

(1) Grain boundary. A grain boundary is the interface between two grains in a polycrystalline material. Ceramics always consist of grains and grain boundaries. If there 
is composition difference between grains and grain boundaries, the interfaces between them will become light scattering sites [64].

(2) Residual pores. Pores are the most major factor that causes light scattering. They act as major scatter centers. Pores are void space (or some pores may include gas inside) formed during fabrication. Pores can be considered to have a different refractive index as the ceramic material. So that it reflects and refracts light. Large pores will cause optical geometry scattering, which can be visible using naked eyes. Rayleigh or Mie scattering occur when pore size is comparable or smaller to wavelength of light, in terms of micrometer or nanometers. But even pore volume of part per million (ppm) will cause quite a large portion of light attenuation and makes ceramics translucent or opaque [65].

(3) Secondary phase. The existence of a secondary phase between the grain boundaries or inside the bulk material will cause non-homogeneity of optical property of material. So maintain the pure phase of material is very important. The amount of sintering additives added should also be kept as low as possible to avoid the generation of the secondary phases.

(4) Double refraction, which is also called birefringence. It's scattering of light when it goes into an anisotropic medium. This is why generally ceramics with a cubic lattice structure can be fully transparent since it is isotropic without birefringence effect. Examples are YAG, sesquioxide [66-68], magnesium aluminate spinel $\left(\mathrm{MgAl}_{2} \mathrm{O}_{4}\right)$ [69] and aluminum oxynitride spinel (ALON) [70].

(5) Impurities such as contaminations or foreign inclusions will also cause the nonhomogeneity of the bulk material. So careful processing and high purity of the sample material is very crucial.

(6) Surface roughness. This is one external factor that affects the ceramic products' transparency. A rougher surface means a higher diffuse scattering, so the sample surface should be processed as smooth as possible. This can be minimized by polishing or 
lapping technology [71].

And the ceramic's total thickness is also another factor affects its transparency. When light passes through the bulk sample's body, the above factors will cause the attenuation of light, and light intensity decreased exponentially as the thickness increases. This typically follows the Beer-Lambert law [72].

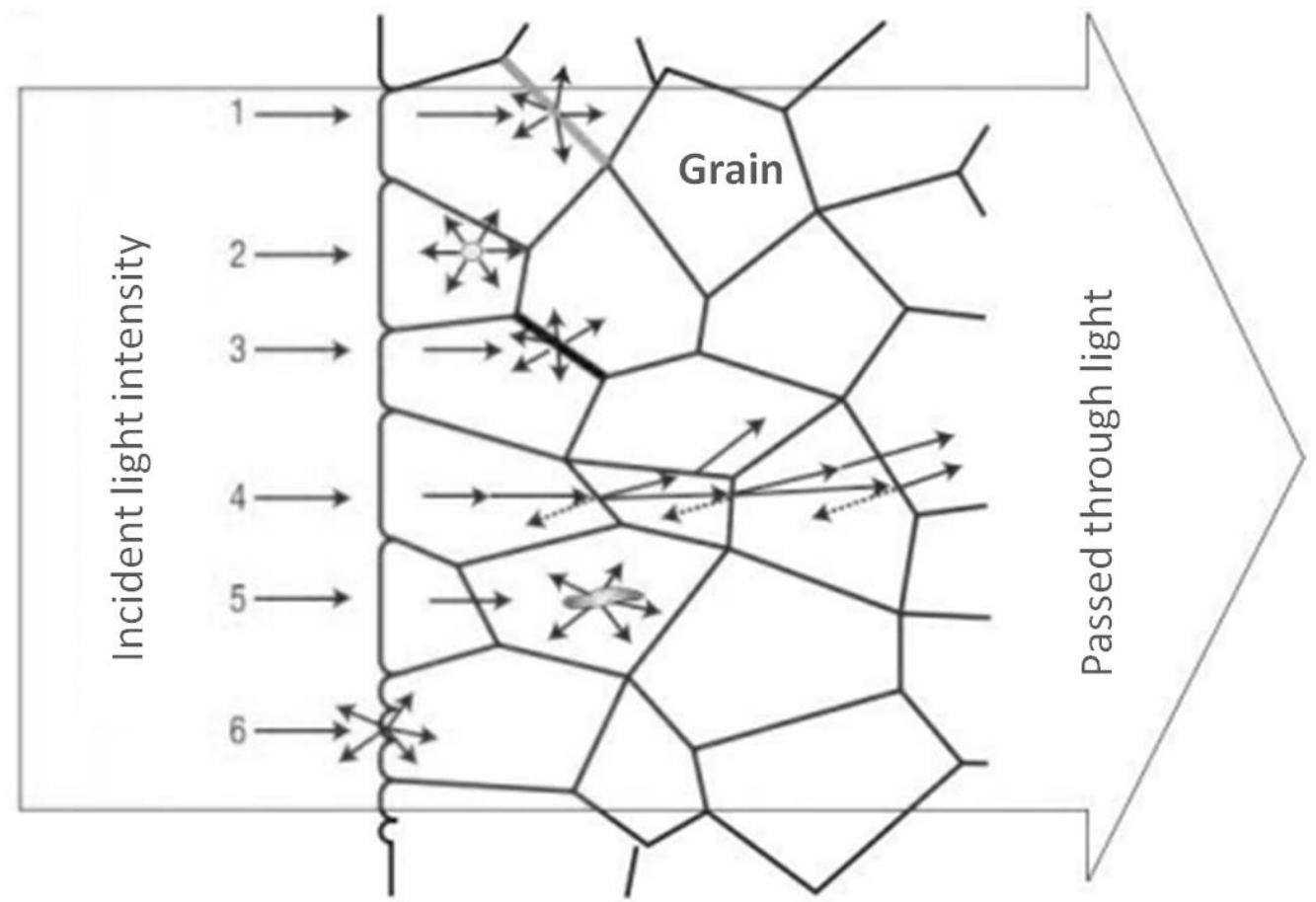

Figure 2.3 Schematic drawing of light scattering factors in polycrystalline ceramics [63].

These factors limit the transparency of ceramic materials. They are originally used in the applications where their transparency can be of tolerance. For example, in the 1960s, Coble et al. at General Electric (GE) Company use thin layer of translucent aluminum oxide in the high pressure sodium-vapor lamps as the discharge tubes material [73]. But still, this opens the possibility that ceramics can be made into optical related applications. By controlling the material purity, improving the sintering process, the light scattering factors can be effectively avoided and ceramics optical transparency can be increased. 
Translucent ceramics also can be used in lighting industry. Wei has studied the properties of transparent ceramic lamp tube materials, which includes small-grained polycrystalline alumina (PCA), sapphire, aluminum oxynitride and YAG [74]. If the ceramic's transparency can reach a higher level, they can also be used as optical observation window, infrared dome, etc. During the past two decades, additional types of transparent ceramics have been developed, include transparent magnesium aluminium spinel, aluminium oxynitride $(\mathrm{AlON})$ and $\mathrm{MgF} 2$ [75]. They are used for the applications such as lenses [76, 77], nose cones for heat-seeking missiles [78], transparent armor windows for military vehicle or aircraft [79-81], scintillation material for computed tomography (CT) scanners [82, 83], and optical attenuator (OA) [84]. The photo of some applications of transparent ceramics is shown in Figure 2.4 [85].

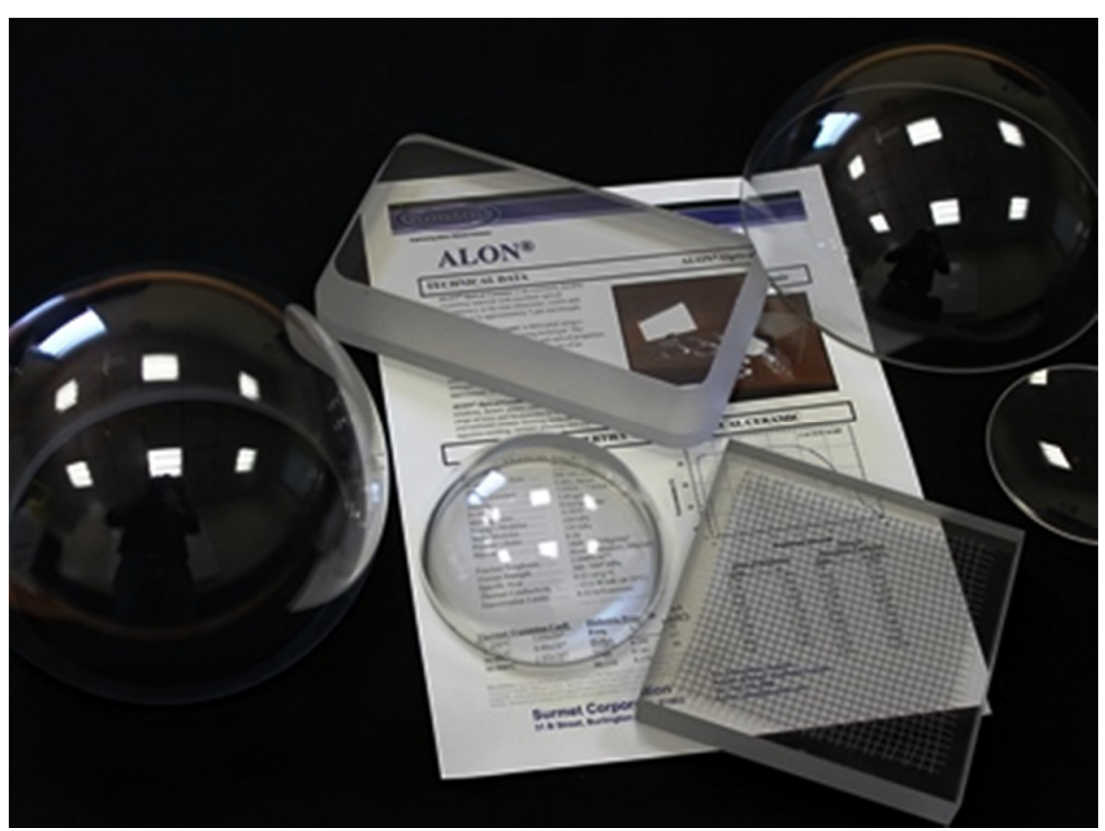

Figure 2.4 Photographs of transparent ceramics with different shapes for different applications [85].

\subsubsection{Laser ceramic materials}

The application of transparent ceramics in laser applications represents the highest standard of material processing technology. It needs the most stringent fabrication 
requirement for the perfect optical quality of transparent ceramics. In the laser system, since the light beam will travel through the laser gain medium repeatedly, any presence of the previously mentioned scattering factors will cause the light attenuate exponentially, which will result a great optical loss. Once the optical loss exceeds the optical gain, the laser oscillation will not occur. For high quality single crystals, the optical loss is quite low, often in the range of $0.3-0.2 \% \mathrm{~cm}^{-1}$ [86], which is sufficiently low enough for laser generation. But the transparent ceramics have a relatively high optical loss. This implies that only by improving the optical quality and reduces the optical loss of transparent ceramics that the laser operations can then be realized.

The first successful laser oscillation of ceramic as laser gain medium was in 1964, by using Dy: $\mathrm{CaF}_{2}$ ceramic under the cryostatic condition [34]. But there's not much information available on the property of the material itself. In 1973, Greskovich et al. from GE company firstly developed a $\mathrm{Nd}: \mathrm{ThO}_{2}-\mathrm{Y}_{2} \mathrm{O}_{3}$ ceramics and successfully achieved lasing demonstration at room temperature with the slope efficiency was only $0.1 \%$ [87]. However the lasing efficiency was too low for practical applications. Due to the technology limit at that time, not many reports have been made and the research on laser ceramics has gone into a low tide period. Almost two decades have passed before the breakthrough was made in 1995 by Ikesue et al. [88]. They obtained the 1.1 at.\% neodymium doped yttrium aluminum garnet (Nd:YAG) laser ceramics by using a solidstate reaction method and high purity powders as raw material. The ceramic sample had the average grain size of $50 \mu \mathrm{m}$ and $99.98 \%$ relative density. Continuous wave laser oscillation was achieved with $70 \mathrm{~mW}$ output power and slope efficiency $28 \%$ [89]. The success of Nd:YAG ceramic laser revived much attention, because now the ceramic materials can perform equally with single crystal materials. And it is a great milestone in the development of ceramic lasers. Since the ceramic processing is much easier and cost lesser than the growth of single crystals, it is hoped that transparent ceramics will be a promising future candidate for solid state lasers.

Compared with the single crystal materials, the polycrystalline laser ceramics have a number of advantages, such as the short production period, lower production cost and the 
flexible size with complicated structures [63]. Since the first demonstration of Nd:YAG ceramic laser in 1995, the research and development on laser ceramics have kept a fast pace. Up till now, various kinds of rare-earth ions doped laser ceramic materials have been successfully developed [90-95]. New laser ceramics such as sesquioxide, which was too difficult to obtain using the conventional crystal growth can also be fabricated by using ceramics fabrication technology [96-98]. For example, by using advanced ceramic technology, composite ceramics with layer-by-layer structure can also be easily fabricated [99-102], whereas it is very difficult to fabricate single crystals with similar structure by using conventional $\mathrm{Cz}$ method. Efforts have also been made to minimize these light scattering factors and improve optical quality of laser ceramics, thus increasing output power of ceramic lasers.

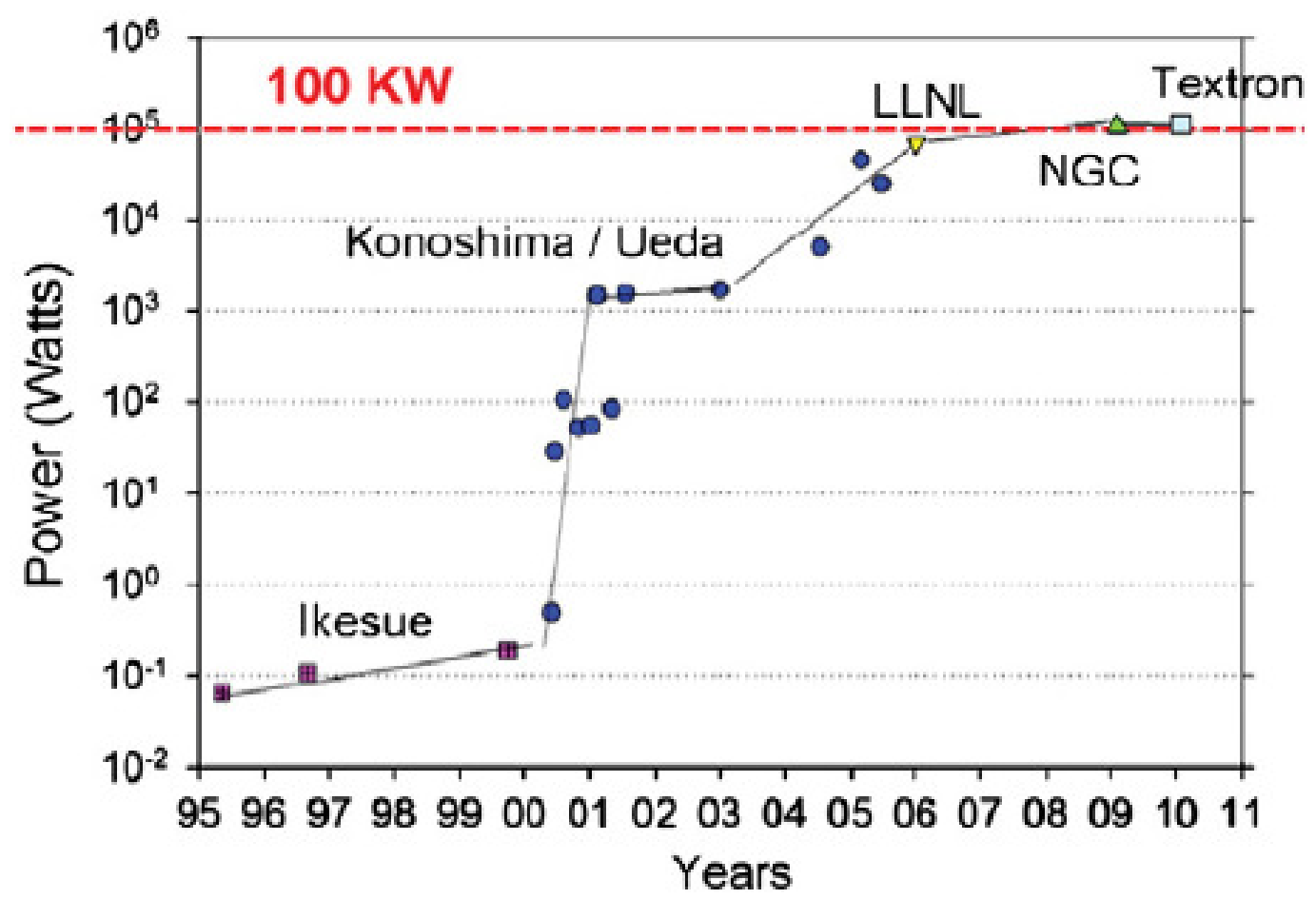

Figure 2.5 Development progresses of ceramics lasers [107,108].

Lu et al. reported an increased laser output power of $357 \mathrm{~mW}$ and achieved a laser slope efficiency of 53\% of Nd:YAG ceramics laser in 2000 [103], The ceramics quality were comparable with that of the single crystal at that time. In 2001, a $1.46 \mathrm{~kW} \mathrm{Nd:YAG}$ 
ceramic laser was demonstrated in Toshiba Corporation in Japan [104], which was a tremendous increase in laser output power. Lu et al. had then developed a $110 \mathrm{~W}$ high power Nd:YAG ceramics laser in 2004, with a laser slope efficiency of $41 \%$ and good beam quality [105]. Since then, higher power ceramic lasers have been continuously reported. In 2006, the Lawrence Livermore National Lab (LLNL) has developed a solid state heat capacity ceramics laser with an output power of 67 KW. In 2009, Northrop Grumman Corp. (NGC) demonstrated a >100 KW end-pumped Yb:YAG slab ceramics laser, followed by a zigzag Nd:YAG ceramic laser developed by Textron company in 2010 [106]. Figure 2.5 shows the development progress of ceramics lasers in the past 20 years $[107,108]$.

Reliability of high quality ceramics as laser gain media is the most important prerequisite for achieving high power and high efficiency lasers. It is still a challenging work for researchers. Yet, compared with the matured growing techniques for single crystal, research on laser ceramics is just at its very beginning phase. There are still many unknown and unsolved scientific questions we need to answer. The detailed fabrication approach for laser ceramics will be explained in the next section.

\subsection{Fabrication approach for laser ceramics}

As briefly mentioned in previous chapter, the fabrication of laser ceramics is no essentially different from other ceramics. Its fabrication is a processing flow including various steps as shown in Figure 2.6. Similar to that of traditional ceramics, the process of transparent ceramics also consists of synthesis of precursor powder, calcinations, shaping and forming, sintering and densification, and post-sintering treatment (such as annealing, machining and polishing). However, the process of transparent ceramics has its stringent requirements and conditions, especially for the powders synthesis and sintering. The detailed fabrication approach will be discussed in this section. 


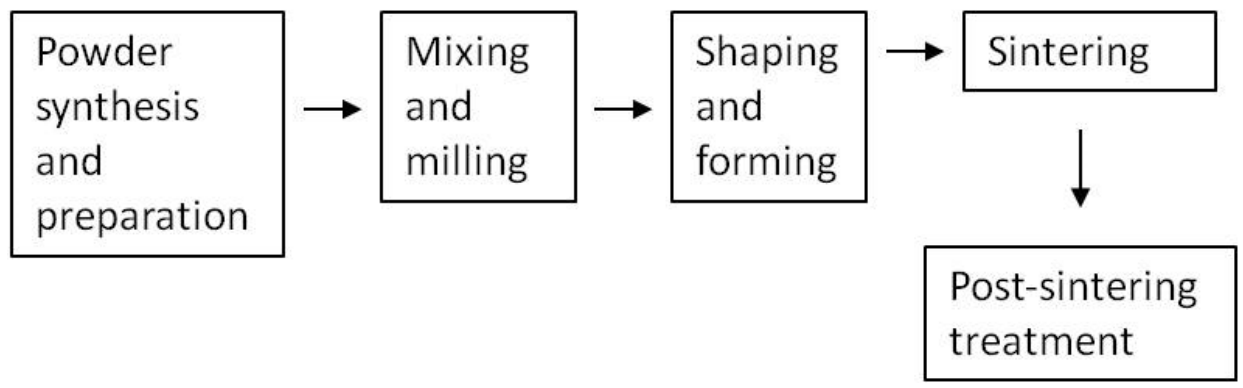

Figure 2.6 General fabrication patterns of laser ceramics.

\subsubsection{Powder synthesis}

To obtain high quality laser ceramics with full density, there are several pre-requirement for the raw powders, such as (1) high purity and controlled size and size distribution, (2) good dispersion and without severe agglomerations, (3) uniform and homogeneity and (4) high sinterablity. There are various methods to synthesize precursor powders of transparent laser ceramics, which are described as follows.

(1) Solid state reaction method [88]. It is mainly used to synthesize multi-component powder mixtures. The materials are typically oxides, carbonates, nitrates, sulphates, etc. The dry powders are mixed together according to the calculated chemical stoichiometric composition and reaction occurs during the process.

(2) Wet chemical co-precipitation method [109-112]. This method is a convenient way to synthesize nanopowders from salt solutions. For example, for multi-component composition, chemical precipitation is performed by the reverse-strike technique, which means the solution of nitrate salts is added drop wise into the precipitant solution. This method has the advantage of higher cation homogeneity in the precursor powder.

(3) Sol-gel process [113-114]. This process involves the formation of a polymeric colloidal suspension (sol) and its gelation to form a continuous network in a liquid phase (gel). The gel will then be calcined to produce oxide powders. The powder particle size can be controlled by the sol/gel transition process. It's very popular for synthesis of 
homogenous nanopowders. Other powder synthesis methods also include hydrothermal method [115-117], spray pyrolysis method [118, 119] and combustion method [120].

\subsubsection{Green body shaping and forming}

Before sintering, the loose powders have to be formed into desired shape to make the bulk body of a final ceramic product. This step is green-body forming or powder compaction. The microstructure of the green body and its relative density has an important effect on the sintering and densification of the final ceramic products. The homogeneous packing of powder and a high packing green body density is desired. The typical green-body shaping methods include dry pressing and cold isotstaic pressing [121, 122], tape casting [123, 124], slip casting [125-127], injection molding method [128, 129] and extrusion method [130]. Some of the shaping methods are summarized in the following Table 2.1 [131].

Table 2.1 Ceramic green body shaping method.

\begin{tabular}{l|l|l|l|l}
\hline Process & Shape & Equipment & Benefits & Drawbacks \\
\hline $\begin{array}{l}\text { Dry } \\
\text { pressing }\end{array}$ & $\begin{array}{l}\text { Uniform } \\
\text { cross- } \\
\text { section }\end{array}$ & Mold or die & $\begin{array}{l}\text { High production } \\
\text { rate, high green } \\
\text { density }\end{array}$ & Simple shape \\
\hline $\begin{array}{l}\text { Tape } \\
\text { casting }\end{array}$ & Thin sheets & $\begin{array}{l}\text { Tape casting } \\
\text { machine }\end{array}$ & $\begin{array}{l}\text { Large area of thin } \\
\text { flat sheets, high } \\
\text { production rates }\end{array}$ & $\begin{array}{l}\text { Tape may crack or } \\
\text { curl due to uneven } \\
\text { drying }\end{array}$ \\
\hline Slip casting & $\begin{array}{l}\text { Various } \\
\text { shape }\end{array}$ & $\begin{array}{l}\text { Porous } \\
\text { separable } \\
\text { mold }\end{array}$ & $\begin{array}{l}\text { Low cost, complex } \\
\text { shape }\end{array}$ & $\begin{array}{l}\text { Low production rate } \\
\text { and lower } \\
\text { dimensional precision }\end{array}$ \\
\hline $\begin{array}{l}\text { Injection } \\
\text { molding }\end{array}$ & $\begin{array}{l}\text { Almost any } \\
\text { shape }\end{array}$ & $\begin{array}{l}\text { Separable } \\
\text { mold }\end{array}$ & Flexible shape & $\begin{array}{l}\text { Longer and slower } \\
\text { debinding time, } \\
\text { expensive equipment }\end{array}$ \\
\hline Extrusion & $\begin{array}{l}\text { Uniform } \\
\text { cross- } \\
\text { section }\end{array}$ & Extruder & $\begin{array}{l}\text { Low cost production } \\
\text { for rods and tubes }\end{array}$ & Easy to deform \\
\hline
\end{tabular}

The different methods will involve either preparation of the powder slurry or direct processing with the dry powders, as well as using different equipment. Different method can be chosen depending on the desired sample's shape/size or application. The finished 
green-body will be ready for the final sintering and densification process.

\subsubsection{Sintering and densification}

Sintering is a processing technique used to consolidate metal or ceramic powder compacts and produce solid dense materials by applying thermal energy in the form of heat [132]. Usually the sintering temperature is below the point of material liquefaction. During this process, any of the remained organic components will be burned out. The contained pores will be expelled so that the porosity is reduced and the compact's volume shrinks and density increases, thus densification occurs. A simple schematic drawing of ceramic sintering process is shown in Figure 2.7.

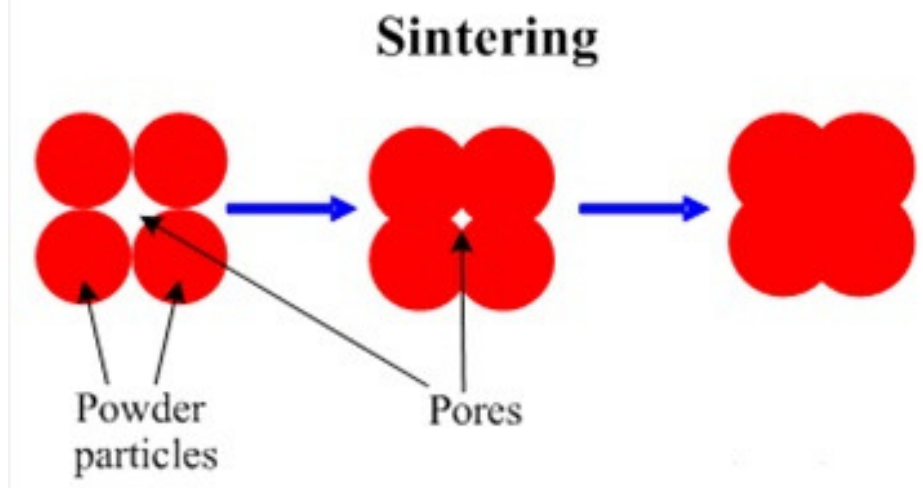

Figure 2.7 Ceramics powder compact sintering process.

Sintering technique is of the oldest technologies used by humans for firing of pottery and porcelains since ancient times. It was only after the 1940s that the fundamental and scientific principles of sintering have been studied. Huge progress and various developments in sintering science have been made ever since then. As one of the most important material synthesis and processing techniques, sintering have become more and more crucial in modern days for materials development. Sintering is also widely used to fabricate powder metallurgical parts as well as bulk ceramic components in industry manufacturing.

Sintering processes can also be divided into mainly of four types: (1) solid-state sintering, 
(2) liquid-phase sintering, (3) viscous flow sintering and (4) vitrification. Figure 2.8 illustrates the different types of sintering in a schematic phase diagram of A-B two components system [133]. Solid state sintering occurs when then powder compact densifies in a total solid state at the sintering temperature T1. Liquid phase sintering occurs for the same powder composition while a liquid phase is present at temperature T3. In between at temperature T2, transient liquid phase sintering happens when there's a combination of both liquid phase sintering and solid state sintering. The liquid phase forms in the compact at the early stage of sintering and disappears as sintering proceeds. The densification is finally completed in the solid state. Viscous flow sintering occurs while the liquid volume fraction is high enough, so that densification of the powder compact can be achieved by a viscous flow. The presence of the liquid phase could promote densification by grain rearrangement and matter can transport faster compared with solid state sintering. The liquid phase sintering also allows the easy control of microstructure and reduction in processing cost, but it degrades some of the important properties of materials such as mechanical properties.

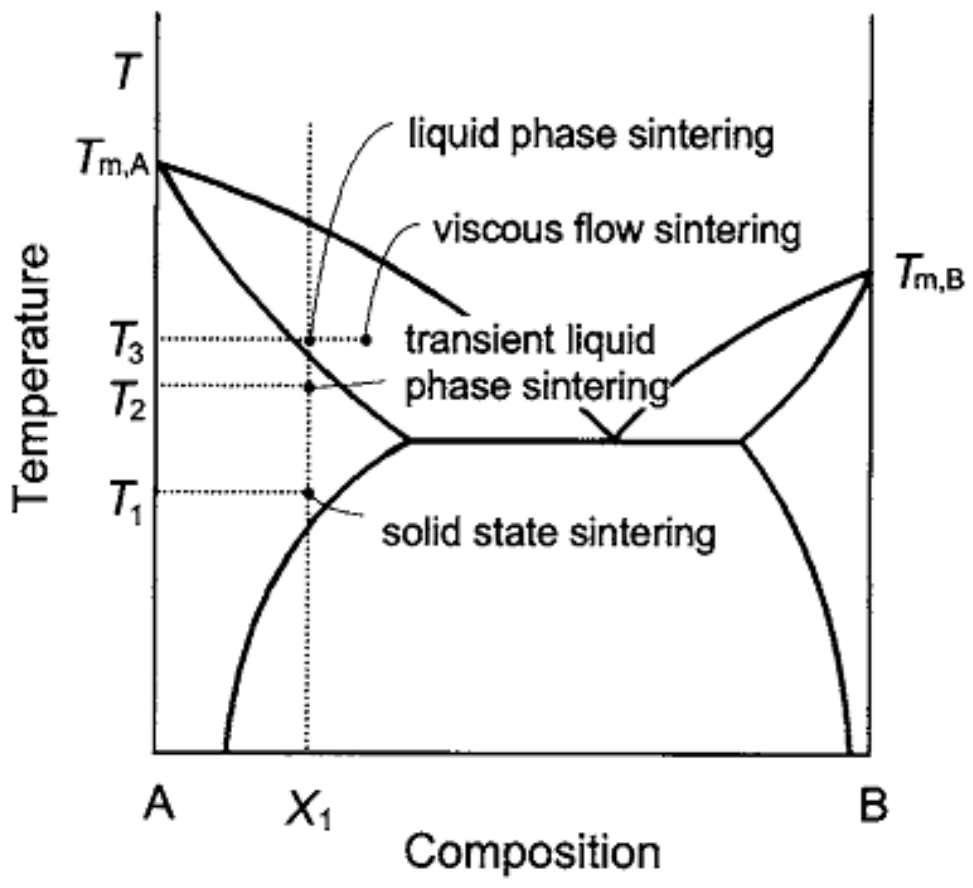

Figure 2.8 Illustrations of different types of sintering. 

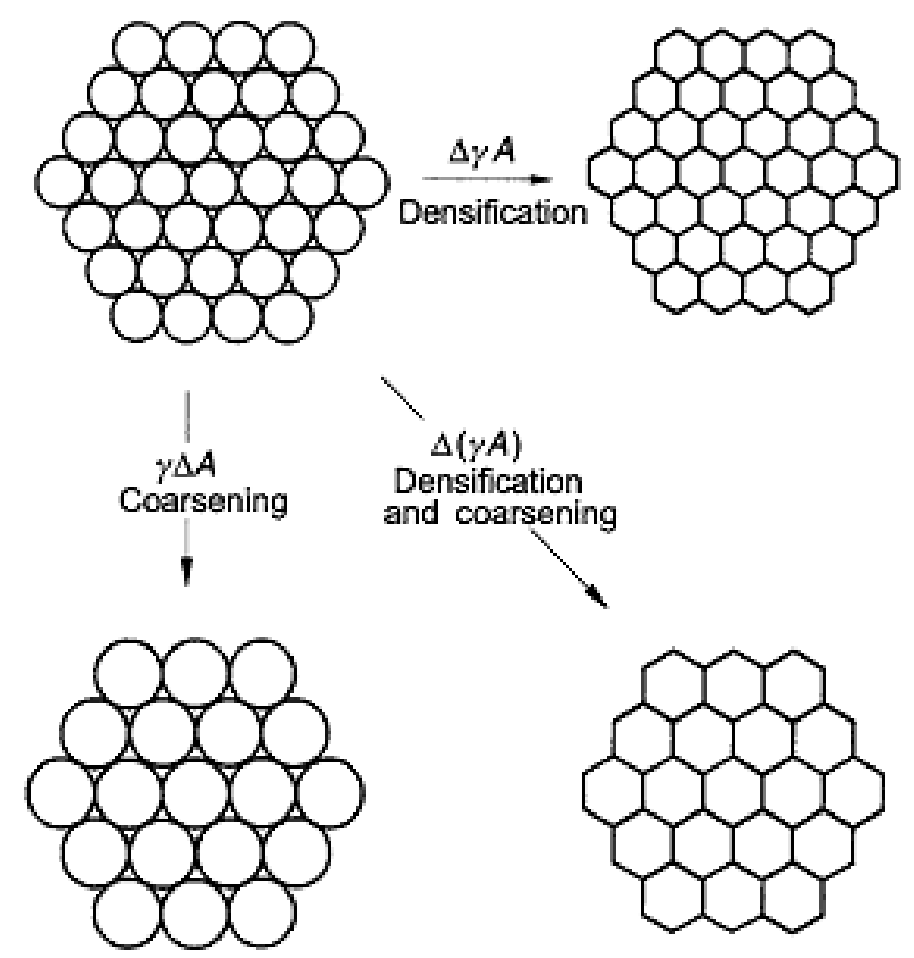

Figure 2.9 Reduction in total interfacial energy through densification and grain coarsening during sintering process.

Sintering process is an irreversible process. The major driving force of sintering is the reduction of the total interfacial energy. Activation energy in the form of heat is required to break the chemical bonds and allow the powder particles to coalesce into single larger one. The total interfacial energy of a powder compact can be expressed as $\gamma \mathrm{A}$, where $\gamma$ is the specific surface energy and $\mathrm{A}$ is the total surface area. So the change in interfacial energy $(\Delta \gamma)$ is due to densification and the change in the interfacial area $(\Delta \mathrm{A})$ is due to grain growth. As shown in Figure 2.9, the reduction in total interfacial energy $(\Delta \gamma \mathrm{A})$ occurs through both densification $(\Delta \gamma \mathrm{A})$ and grain coarsening $(\gamma \Delta \mathrm{A})$ [133].

\subsubsection{Sintering mechanisms}

The matter transport during the ceramics sintering process occurs through diffusion, which is a thermally activated process. The atoms diffuse through different paths in the bulk solid, relating to the different diffusion mechanisms. The chemical potential gradient 
causes the diffusion: atoms are transported from the area of higher chemical potential which is known as the source, to the area of lower chemical potential which is known as the sink. These different paths that the atoms take to get from one region to another are regarded as the sintering mechanisms correspondingly. Because of these different matters transport paths as well as the presence of grain boundaries, ceramics materials sintering phenomena is more complicated than that in the amorphous materials.

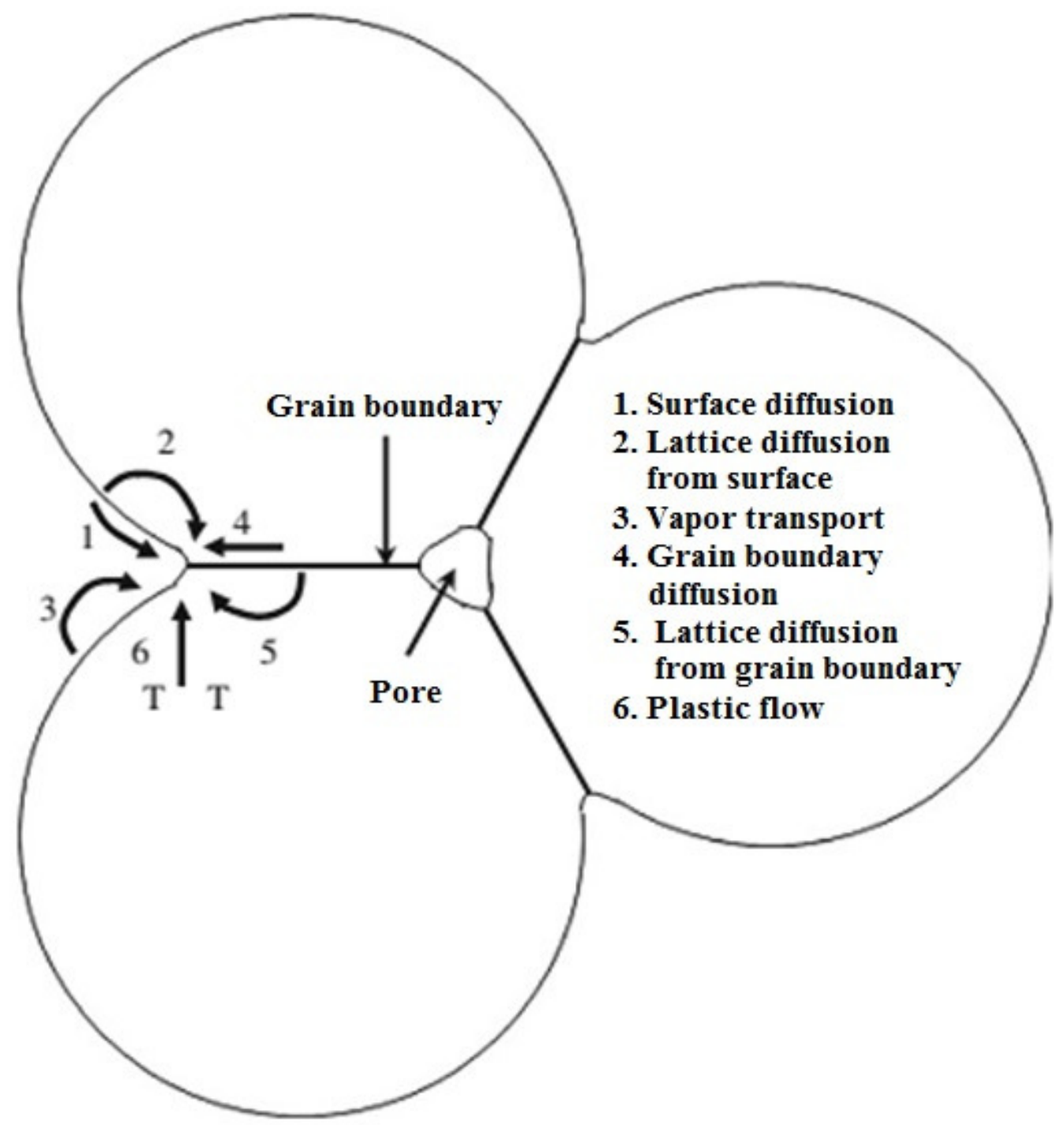

Figure 2.10 Six mechanisms contributing to the sintering of crystalline ceramics. 
The matter transport of the ceramics sintering can take six different paths that determine the sintering mechanisms. Figure 2.10 shows the six mechanisms which contribute to the sintering of crystalline ceramics [134]. They are summarized as follows:

(1) Surface diffusion. It is the diffusion of atoms along a particle surface. The free surface of a particle often contains vacancies and adatoms. The migration and movement of vacancies and adatoms promote the mechanisms of surface diffusion. This diffusion process is usually close to the surface with a layer of thickness of less than $1 \mathrm{~nm}$.

(2) Lattice diffusion from surface. It is the diffusion of atoms through lattice from the particle surface. Lattice diffusion is due to the movement of point defects of the crystal lattice. It consists of different types of mechanism, such as vacancy mechanism, interstitial mechanism, interstitial mechanism and direct exchange mechanism.

(3) Vapor transport. It is the evaporation of atoms from sphere surfaces and condensation on the neck area.

(4) Grain boundary diffusion. It is the diffusion of atoms along the grain boundary area. The grain boundaries separate the crystal grains from each other. There is lattice mismatching and disordering along the grain boundary. The grain boundary diffusion has faster diffusion rate than lattice diffusion in the near grains due to their defective nature.

(5) Lattice diffusion from grain boundary. It is the diffusion of atoms through lattice from the grain boundary.

(6) Plastic flow. It is due to the dislocation motion which causes the flow of matter.

All these mechanisms can be divided into densifying mechanisms as well as nondensifying mechanisms. It depends on whether it will cause the shrinkage of volume or densification of the powder compact. The non-densifying mechanisms include mechanisms 1, 2 and 3, i.e., surface diffusion, lattice diffusion from the particle surfaces 
and vapor transport. These mechanisms only lead to neck growth and reduce the curvature of the neck surface but without densification. The other mechanisms 4 and 5 , grain boundary diffusion and lattice diffusion (where grain boundary is the source) are densifying mechanisms. They contribute most importantly to the densification of ceramics. Diffusion from the grain boundary favours neck growth and densification. Plastic flow also causes the neck growth and densification, and it is more obvious during the metal powders sintering. Viscous flows and particle deformation causes the neck growth and densification of amorphous materials which have no grain boundaries such as glasses. In practical situations, the paths of matter transport are actually very complex. The sintering mechanisms of materials transport are summarized in Table 2.2.

Table 2.2 Material transport mechanisms of sintering.

\begin{tabular}{l|l|l|l}
\hline Mechanisms & Material source & Material sink & \\
\hline 1. Surface diffusion & Surface & Neck & Nondensifying \\
\hline 2. Lattice diffusion from surface & Surface & Neck & Nondensifying \\
\hline 3. Vapor transport & Surface & Neck & Nondensifying \\
\hline $\begin{array}{l}\text { 4. Grain boundary diffusion } \\
\text { 5. Lattice diffusion from grain } \\
\text { boundary }\end{array}$ & Grain boundary & Neck & Densifying \\
\hline 6. Plastic flow & Grain boundary & Neck & Densifying \\
\hline
\end{tabular}

\subsubsection{Sintering stages}

The sintering process can also be categorized into three stages in sequence: (1) the initial stage, (2) the intermediate stage and (3) the final stage. Some researchers also include an extra "zero stage" to determine the moment of the instantaneous contact of the powder particles. The elastic deformation brought the particles together due to the reduction of surface energy at interfaces [135]. A stage represents a period section of time during which the microstructure of powder compact is well defined. Figure 2.11 shows the 
idealized model for the three stages of sintering of polycrystalline materials [136, 137].

Initial Stage

The initial stage begins with powder particle spheres contact tangentially. It is characterized by neck formation and rapid neck growth between particles through diffusion, vapor transport and viscous flow, as shown schematically in Figure 2.11 (a). The compact volume shrinkage and densification could occur when the neck growth is dominated by densifying mechanisms. The initial surface curvature differences between particles will be eliminated at this stage. Towards the end of the initial stage, particle spheres begin to coalesce as shown in Figure 2.11 (b). The radius of the neck between the particles can reach about half of the particle radius. For the green body with a starting density of $50 \%$ $60 \%$ of the theoretical density, the relative density can increase for about $3 \% \sim 5 \%$ during this sintering stage.

Intermediate Stage

During the intermediate stage, the pores begin to form their equilibrium shapes because of the surface tension. The grains have formed the tetrakaidecahedron shape with continuous pores forming interconnected channels along the grain edges as shown in Figure 2.11 (c). Densification also takes place because of the shrinkage of the pores. The pores are pinched off finally and those isolated pores are remained in the sintered compact before the final stage starts. The intermediate stage contributes the main process of sintering. The compact relative density can increase to about $90 \%$ of the theoretical density. 

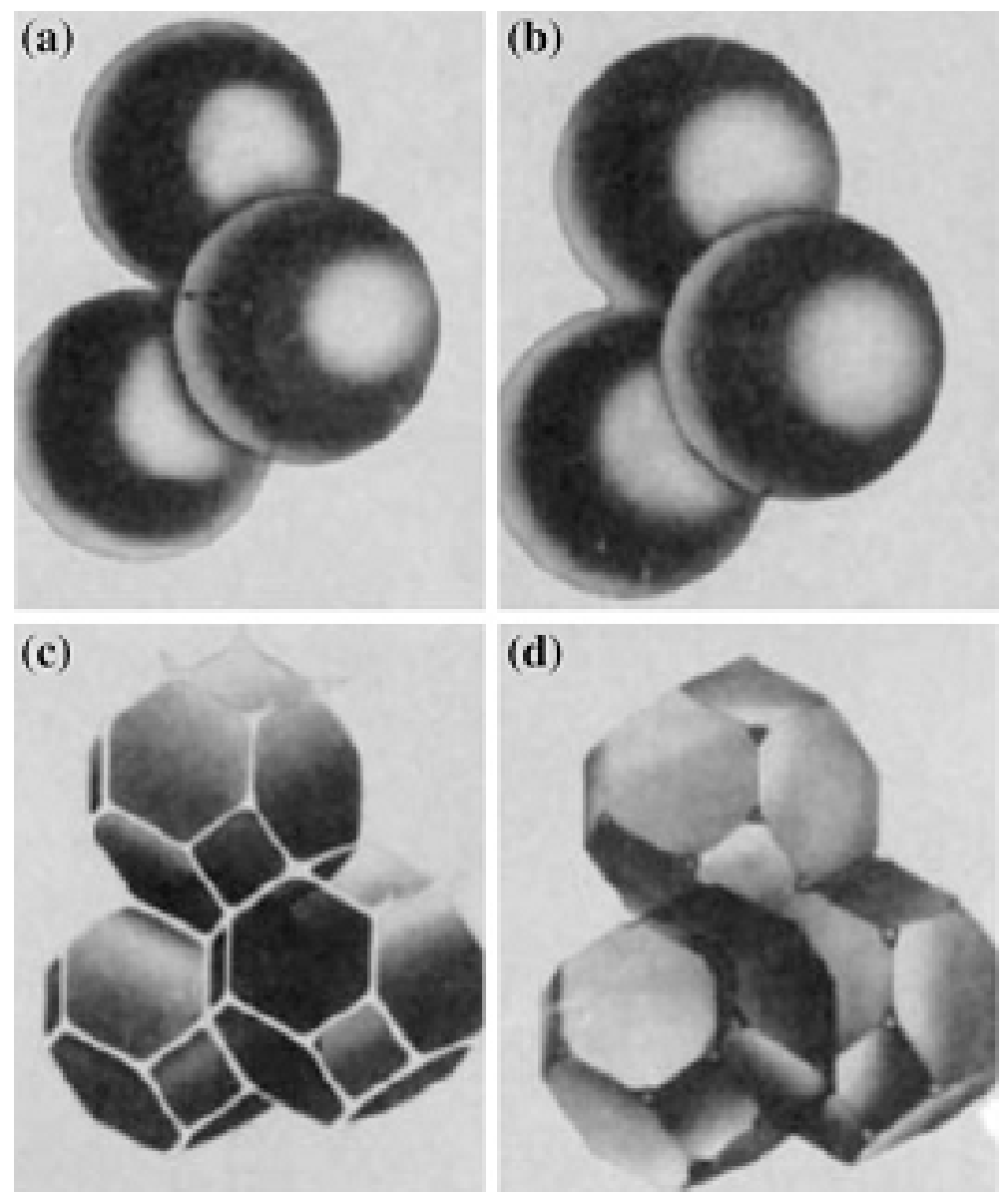

Figure 2.11 Idealized models for the three stages of sintering: (a) Initial stage, (b) Towards the end of initial stage, (c) Intermediate stage and (d) Final stage.

\section{Final Stage}

In the final stage, the pores are expelled and only isolated pores of tetrahedral inclusions located at grains corners, which is schematically in Figure 2.11 (d). The pores will shrink continuously and finally disappear in the end. The density of compact will increase to almost close to $100 \%$ of the theoretical density of the material. And thus sintering process is complete. Figure 2.12 shows the schematic drawing of the densification curve of a green body compact and the sintering stage with respect to the increase of sintering time [133]. 


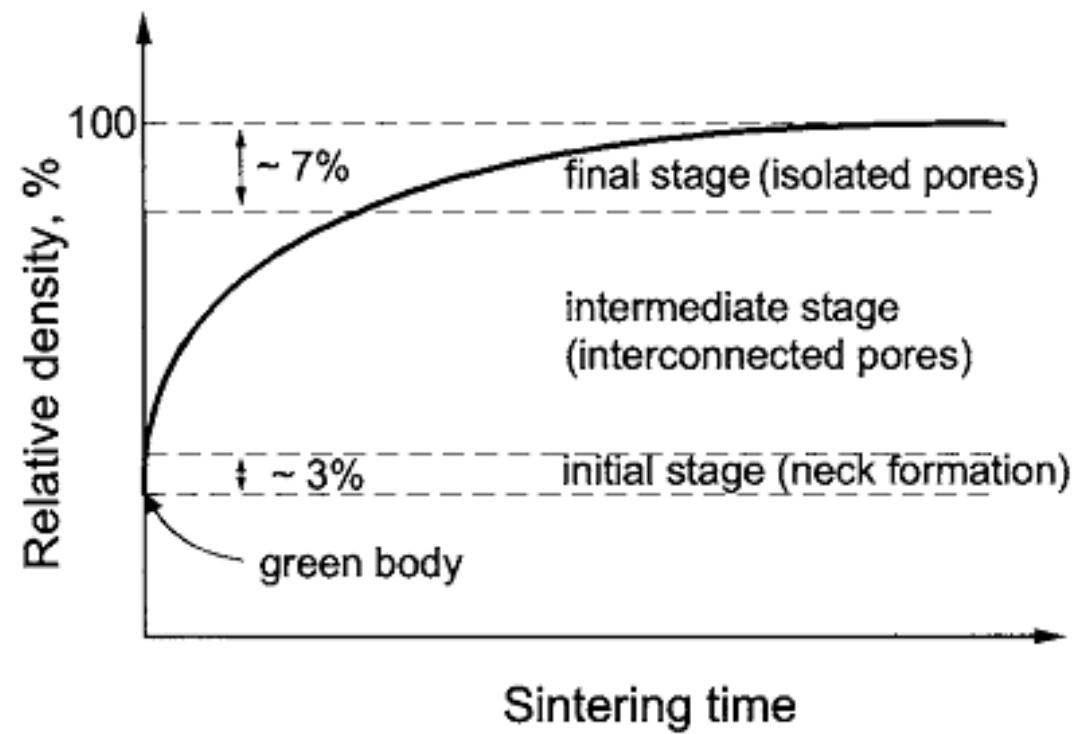

Figure 2.12 Schematic drawing of the densification curve for a powder compact and the three sintering stages.

In practical sintering experiments, there're various processing variables and adjustable parameters that can determine sinterability and sintered properties of powder compact samples. They can be divided into the variables related to raw material itself and the parameters related to sintering process conditions. The material variables include powder shape, size, size distribution, the level of agglomeration, the degree of mixing uniformity, as well as powder's chemical composition, purity, stoichiometry, etc. These variables will influence the powder sinterability. So typically the mechanical ball milling process or chemical process such as co-precipitation are used to improve powder homogeneity. The sintering parameters include sintering temperature, time, atmosphere, pressure, heating and cooling rate. Combined with all these variable and parameters, sintering is in fact a quite complicated process. Many studies have also been focused on how to precisely control these variable and parameters. In practice, the various sintering technologies will involve quite a lot of complicated and specially designed equipments. Some major sintering techniques are summarizes in the following part:

(1) Vacuum sintering [88]. Vacuum sintering is a sintering processes carried out in 
vacuum sintering furnace at a relative high vacuum. It can achieve better sintering results compared with those run at normal pressure. Vacuum sintering can typically be used to fabricate garnet or sesquioxide laser ceramics [138, 139].

(2) Hot press (HP) sintering [140, 141]. This is a process when heat and pressure (50-100 $\mathrm{MPa}$ ) are simultaneous applied to the samples in the hot press furnace. High pressure can promote plastic flow and particle rearrangement, thus enhance densification.

(3) Hot isostatic pressing (HIP) [142-145]. This sintering technique can reduce the porosity and further increase density by applying high temperature and high pressure to the material from all directions. Specially designed HIP furnace is required for the sintering experiments to withstand high temperature and high pressure.

(4) Spark plasma sintering (SPS) [146-148]. It is a sintering method by using high density current flux to flow through the sample and the carbon die, to cause the heating within the sample body. Because it can achieve densification within a short time, so it can obtain samples without the presence of significant grain growth.

(5) Microwave sintering [149-152]. This method can let the processed materials absorb microwave power and then convert microwave energy to heat within the material itself. It has the advantages of higher energy efficiency, enhanced reaction and sintering rate, short cycle time and cost savings. Microwave sintering can be conducted at lower sintering temperatures and much shorter sintering times.

\subsection{Challenges in transparent laser ceramics}

As early as in the 1980s, much of the pioneering efforts have been devoted to fabrication of high quality un-doped YAG and rare-earth ions doped YAG transparent ceramics for high-temperature's window, arc tubes for lamp and laser materials. In 1990, M. Sekita reported the optical properties of $\mathrm{Nd}$ :YAG ceramics fabricated from a urea precipitation method [153]. Optical properties of the sample are almost the same as those of single 
crystals grown by the $\mathrm{Cz}$ method and floating-zone method, but the background absorption is up to $2.5-3.0 \mathrm{~cm}^{-1}$, which was not good enough for laser experiment. The major breakthrough was in 1995, when Ikesue et al. reported first laser oscillation using transparent Nd:YAG ceramics. The ceramics were fabricated by solid-state reactive sintering technology using high-purity powders (>99.99 wt.\% purity) as starting materials. Tetraethyl orthosilicate (TEOS) was added as a sintering additives [88]. The mixture was ball-milled using high-purity alumina media in ethanol solvent. After well mixing, the slurry was dried in oven. Then the powder was pressed into various shapes. The shaped sample was then cold isostatically pressed at $200 \mathrm{MPa}$, and a green body with a packing density of $50-55 \%$ of theoretical density was obtained. After calcinations at about $800^{\circ} \mathrm{C}$ to remove the organic component, the powder compact was sintered under vacuum conditions. During the sintering, YAG phase crystallite was formed and fully dense ceramics were obtained with the grain growth as well as density increase. The samples were then optical polished to remove surface roughness. The fabrication process is illustrated in Figure 2.13.

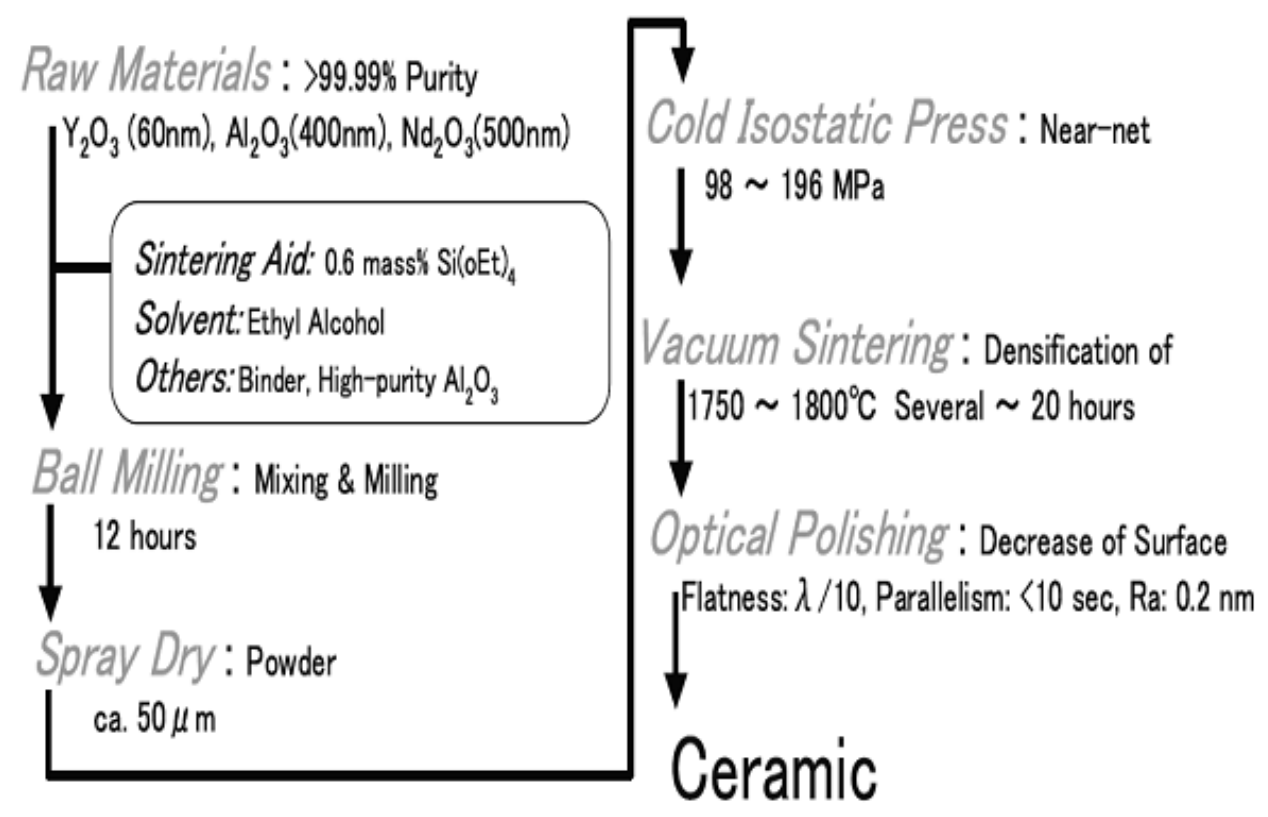

Figure 2.13 Fabrication process for Nd:YAG ceramics by solid-state reactive sintering. 


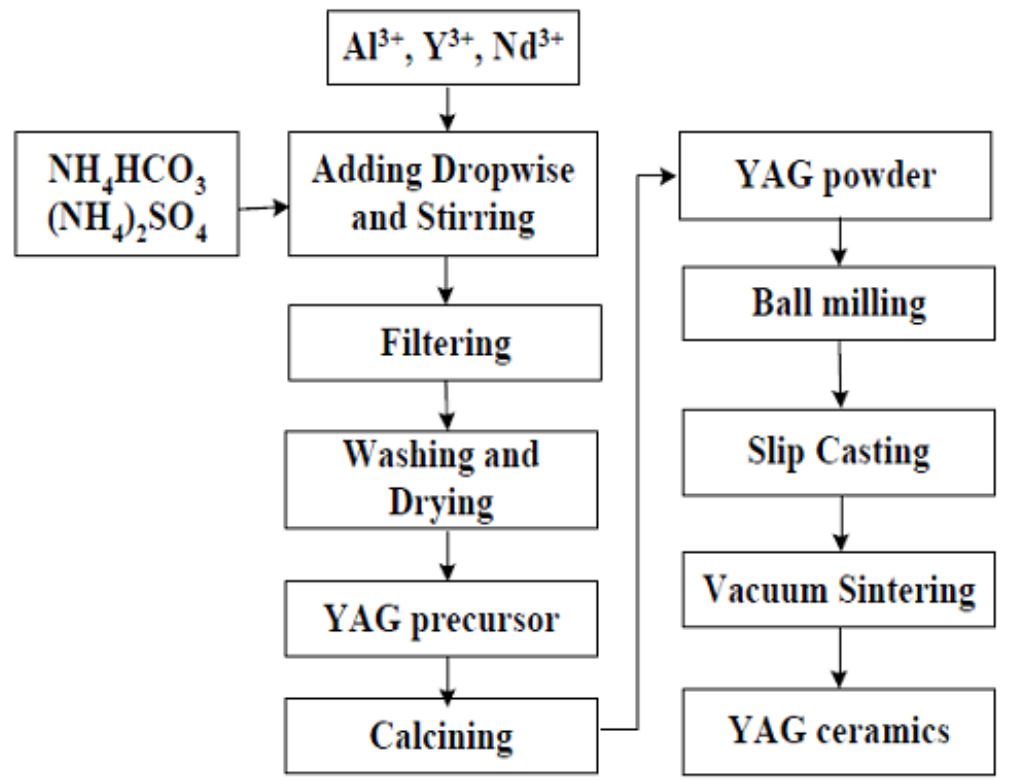

Figure 2.14 Fabrication process for Nd:YAG ceramics by a wet chemical method.

Since 1999, Ueda's group in University of Electro-communication and Yagi's group in Konoshima Corp. Ltd began to fabricate highly transparent ceramic laser materials by a wet chemical method as shown in Figure 2.14 [104]. In this process, yttrium, aluminum and neodymium chloride solutions of were used as source materials to prepare high quality YAG precursors first. Then the compact pellets were sintered under high vacuum conditions. Scattering losses were greatly reduced by using the improved wet-chemical powder synthesis and the slip casting formation technique.

The advantages of using ceramic as laser materials lie in the fabrication process. Such as short period Compared with single crystal laser materials, polycrystalline ceramic laser materials have the following advantages. The advantages include:

(1) Relative ease of fabrication.

(2) Less expensive, lower fabrication cost.

(3) Higher doping concentration.

(4) Fabrication of large size samples.

(5) Multi-layer and multi-functional ceramic structure. 
The current problems and challenging for obtaining real good quality laser ceramics still lies in each stage of the ceramics fabrication process. Starting from raw powders control to the final sintering condition control. The sinterability of raw powder also depends on the powder characteristics, including the purity, homogeneity, particle average size, size distribution and particle shape. The conditions of each subsequent stage also depend on the quality of the products of the previous stage.

\subsection{PhD in context of literature}

By addressing the previous problems and challenges, this $\mathrm{PhD}$ study mainly focus on establishing the most cost-effective fabrication method to obtaining the high quality ytterbium doped laser ceramics which are suitable for practical laser applications. New types of laser ceramics materials are being developed based on the established fabrication method. High power solid state laser systems are built and high efficiency laser performances are demonstrated. And thus leads to the following novel outcomes:

(1) The fabrication techniques for $\mathrm{Yb}$ doped $\mathrm{YAG}$ garnet laser ceramics were established. Successful laser operation was achieved by using the in-house fabricated samples.

(2) Yb doped GdYAG mixed garnet laser ceramics were obtained by similar method with laser performances demonstrated.

(3) Yb doped LuAG garnet laser ceramics were obtained and reported for the first time. $\mathrm{CW}$ and femtosecond laser operations were also demonstrated.

The rationale for materials selection and experimental method will also be elaborated in detail in the next chapter. 


\section{References}

[1] W. Koechner, Solid-state laser engineering. Springer, 2013.

[2] O. Svelto, and D. C. Hanna, Principles of lasers, Springer, 1976.

[3] W. Koechner, and M. Bass, Solid-State Lasers: A Graduate Text. Springer Science \& Business Media, 2003.

[4] T. O. Hardwell, Solid-state Lasers: Properties and Applications, Nova Publishers, 2008.

[5] T. Maiman, Physical Review Letters 1960, 4, 564.

[6] T. H. Maiman, Nature. 1960, 187, 493-494.

[7] H. Xu, and X. Wang, Infrared and Laser Engineering 2007, 36, 13.

[8] F. Schwarz, A. Aoki, J. Becker, and A. Sculean, Journal of Clinical Periodontology. 2008, 35, 29-44.

[9] K. Tsubota, Ophthalmologica 1990, 200, 117-122.

[10] B. Finkel, Y. Eliezri, A. Waldman, and M. Slatkine, Journal of Clinical Laser Medicine \& Surgery. 1997, 15, 225-229.

[11] J. Ion, Laser processing of engineering materials: principles, procedure and industrial application, Butterworth-Heinemann, 2005.

[12] L. J. Radziemski, and D. A. Cremers, Laser-induced plasmas and applications. Marcel Dekker Inc., 1989.

[13] M. C. Gower, Optics Express. 2000, 7, 56-67.

[14] G. P. Agrawal, and N. K. Dutta, in Semiconductor Lasers, Springer 1993, 547-582.

[15] C. Henry, IEEE Journal of Quantum Electronics. 1982, 18, 259-264.

[16] K. Iga, F. Koyama, and S. Kinoshita, IEEE Journal of Quantum Electronics. 1988, 24, 1845-1855.

[17] K. Tomiyasu, The Laser Literature, Springer 1968, 24-30.

[18] K. Seta, and T. O'ishi, Applied Optics. 1990, 29, 354-359.

[19] W. B. Tiffany, R. Targ, and J. Foster, Applied Physics Letters. 1969, 15, 91-93.

[20] S.-L. Chen, Journal of materials processing technology. 1998, 73, 147-159.

[21] F. J. Duarte, P. Kelley, L. W. Hillman, and P. F. Liao, Dye laser principles, Academic Press, 1990. 
[22] D. Moses, Applied Physics Letters. 1992, 60, 3215-3216.

[23] S. A. Payne, L. Chase, H. W. Newkirk, L. K. Smith, and W. F. Krupke, IEEE Journal of Quantum Electronics. 1988, 24, 2243-2252.

[24] R. BYER, Science 1988, 239, 742-747.

[25] N. Sarukura, Y. Ishida, T. Yanagawa, and H. Nakano, Applied Physics Letters 1990, 57, 229-230.

[26] E. C. Honea, R. J. Beach, S. B. Sutton, J. A. Speth, S. C. Mitchell, J. A. Skidmore, M. A. Emanuel, and S. A. Payne, IEEE Journal of Quantum Electronics. 1997, 33, 1592-1600.

[27] J. Geusic, H. Marcos, and L. Van Uitert, Applied Physics Letters 1964, 4, 182-184.

[28] P. Yang, P. Deng, J. Xu, and Z. Yin, Journal of Crystal Growth. 2000, 216, 348-351.

[29] Y. Shimony, Y. Kalisky, H. Lotem, Z. Goldbart, and J. Kagan, Journal of Applied Physics. 1990, 68, 2966-2971.

[30] J. Carruthers, M. Kokta, R. Barns, and M. Grasso, Journal of Crystal Growth. 1973, 19, 204-208.

[31] P. Schiffer, A. Ramirez, D. Huse, P. Gammel, U. Yaron, D. Bishop, and A. Valentino, Physical Review Letters. 1995, 74, 2379.

[32] W. Krupke, M. Shinn, J. Marion, J. Caird, and S. Stokowski, Journal of the Optical Society of America B. 1986, 3, 102-114.

[33] E. V. Zharikov, N. N. Il'ichev, V. Laptev, A. A. Malyutin, V. Ostroumov, P. P. Pashinin, A. Pimenov, V. A. Smirnov, and I. A. Shcherbakov, Soviet Journal of Quantum Electronics. 1983, 13, 82.

[34] S. Hatch, W. Parsons, and R. Weagley, Applied Physics Letters. 1964, 5, 153-154.

[35] D. Welford, and P. F. Moulton, Optics Letters. 1988, 13, 975-977.

[36] W. D. SCOTT, Journal of the American Ceramic Society. 1962, 45, 586-587.

[37] R. Fields, M. Birnbaum, and C. Fincher, Applied Physics Letters. 1987, 51, 1885 1886.

[38] H. Zhang, X. Meng, L. Zhu, P. Wang, X. Liu, Z. Yang, J. Dawes, and P. Dekker, Physica Status Solidi (a). 1999, 175, 705-710.

[39] J. Chen, F. Guo, N. Zhuang, J. Lan, X. Hu, and S. Gao, Journal of Crystal Growth. 2002, 243, 450-455. 
[40] C. Wang, Y. Chow, L. Reekie, W. Gambling, H. Zhang, L. Zhu, and X. Meng, Applied Physics B. 2000, 70, 769-772.

[41] T. S. Alster, Journal of the American Academy of Dermatology. 1995, 33, 69-73.

[42] R. Scheps, B. M. Gately, J. F. Myers, J. S. Krasinski, and D. F. Heller, Applied Physics Letters. 1990, 56, 2288-2290.

[43] H. R. Verdun, L. M. Thomas, D. M. Andrauskas, T. McCollum, and A. Pinto, Applied Physics Letters. 1988, 53, 2593-2595.

[44] V. Petričević, S. Gayen, R. Alfano, K. Yamagishi, H. Anzai, and Y. Yamaguchi, Applied Physics Letters. 1988, 52, 1040-1042.

[45] M. Ledig, E. Heumann, D. Ehrt, and W. Seeber, Optical and Quantum Electronics. 1990, 22, S107-S122.

[46] W. Seeber, D. Ehrt, and H. Ebendorff-Heidepriem, Journal of Non-crystalline Solids. 1994, 171, 94-104.

[47] J. Czochralski, Z. Phys. Chemie. 1918, 92, 219-221.

[48] G. K. Teal, and J. Little, presented at Physical Review. 1950, 78, 647.

[49] X. Xu, Z. Zhao, G. Zhao, P. X. Song, J. Xu, and P. Deng, Journal of Crystal Growth. 2003, 257, 297-300.

[50] Y. D. Zavartsev, V. Vlasov, S. Kutovoi, A. Zagumennyi, and A. Zerrouk, Journal of Crystal Growth. 2011, 320, 36-40.

[51] X. Fu, Z. Jia, Y. Li, D. Yuan, C. Dong, and X. Tao, Journal of Crystal Growth. 2012, $353,72-76$.

[52] M. Weber, M. Bass, K. Andringa, R. Monchamp, and E. Comperchio, Applied Physics Letters. 1969, 15, 342-345.

[53] H. Tang, H. Li, and J. Xu, Growth and development of sapphire crystal for LED applications, INTECH Open Access Publisher, 2013.

[54] C. Belouet, Journal of Crystal Growth. 1972, 15, 188-194.

[55] W. D. Kingery, Introduction to ceramics. Wiley. 1960.

[56] F. Singer, Industrial ceramics, Springer, 2013.

[57] S. Sasaki, Wear. 1989, 134, 185-200.

[58] A. De Aza, J. Chevalier, G. Fantozzi, M. Schehl, and R. Torrecillas, Biomaterials. 2002, 23, 937-945. 
[59] K. Negita, Journal of the American Ceramic Society. 1986, 69, 308-310.

[60] P. F. Manicone, P. R. Iommetti, and L. Raffaelli, Journal of Dentistry. 2007, 35, 819-826.

[61] T. Furukawa, K. Ishida, and E. Fukada, Journal of Applied Physics. 1979, 50, 49044912.

[62] A. I. Kingon, and J. B. Clark, Journal of the American Ceramic Society. 1983, 66, 253-256.

[63] A. Ikesue, and Y. L. Aung, Nature photonics. 2008, 2, 721-727.

[64] A. Ikesue, K. Yoshida, T. Yamamoto, and I. Yamaga, Journal of the American Ceramic Society. 1997, 80, 1517-1522.

[65] A. Ikesue, and K. Yoshida, Journal of Materials Science 1999, 34, 1189-1195.

[66] K. Takaichi, H. Yagi, A. Shirakawa, K. Ueda, S. Hosokawa, T. Yanagitani, and A. Kaminskii, Physica Status Solidi (a). 2005, 202, R1-R3.

[67] J. Lu, J. Lu, T. Murai, K. Takaichi, T. Uematsu, K. Ueda, H. Yagi, T. Yanagitani, and A. A. Kaminskii, Japanese Journal of Applied Physics 2001, 40, L1277.

[68] J. Lu, J. Bisson, K. Takaichi, T. Uematsu, A. Shirakawa, M. Musha, K. Ueda, H. Yagi, T. Yanagitani, and A. Kaminskii, Applied Physics Letters. 2003, 83, 1101.

[69] C. Wang, and P. Zanzucchi, Journal of the Electrochemical Society. 1971, 118, 586591.

[70] J. W. McCauley, and N. D. Corbin, Journal of the American Ceramic Society. 1979, $62,476-479$.

[71] T. Doi, E. Uhlmann, and I. D. Marinescu, Handbook of Ceramics Grinding and Polishing, William Andrew, 2015.

[72] D. Swinehart, Journal of Chemical Education. 1962, 39, 333.

[73] R. Coble, American Ceramic Society Bulletin. 1959, 38, 507-510.

[74] G. Wei, Journal of the European Ceramic Society. 2009, 29, 237-244.

[75] T. Yen, Y. Chang, D. Yu, F. Yen, D. Tsai, and I.-N. Lin, Materials Science and Engineering: A. 1991, 147, 121-128.

[76] P. Hartmann, H. F. Morian, and R. Jedamzik, presented at Large Lenses and Prisms 2002.

[77] U. Peuchert, Y. Okano, Y. Menke, S. Reichel, and A. Ikesue, Journal of the 
European Ceramic Society. 2009, 29, 283-291.

[78] F. Monteverde, A. Bellosi, and L. Scatteia, Materials Science and Engineering: A 2008, 485, 415-421.

[79] J. J. Swab, and A. Gilde, presented at 23nd Annual Conference on Composites, Advanced Ceramics, Materials, and Structures-B: Ceramic Engineering and Science Proceedings, 2009.

[80] P. J. Patel, G. A. Gilde, P. G. Dehmer, and J. W. McCauley, presented at International Symposium on Optical Science and Technology, 2000.

[81] J. A. Salem, Journal of the American Ceramic Society. 2013, 96, 281-289.

[82] C. Greskovich, and S. Duclos, Annual Review of Materials Science. 1997, 27, 69-88.

[83] Y. Ito, H. Yamada, M. Yoshida, H. Fujii, G. Toda, H. Takeuchi, and Y. Tsukuda, Japanese Journal of Applied Physics. 1988, 27, L1371.

[84] H. Jiang, Y. Zou, Q. Chen, K. Li, R. Zhang, Y. Wang, H. Ming, and Z. Zheng, presented at Photonics Asia, 2005.

[85] http://www.surmet.com/technology/alon-optical-ceramics/.

[86] L. B. Kong, Y. Huang, W. Que, T. Zhang, S. Li, J. Zhang, Z. Dong, and D. Tang, Transparent Ceramics, Springer, 2015.

[87] C. Greskovich, and J. Chernoch, Journal of Applied Physics. 1973, 44, 4599-4606.

[88] A. Ikesue, I. Furusato, and K. Kamata, Journal of the American Ceramic Society. 1995, 78, 225-228.

[89] A. Ikesue, T. Kinoshita, K. Kamata, and K. Yoshida, Journal of the American Ceramic Society. 1995, 78, 1033-1040.

[90] H. Yagi, T. Yanagitani, K. Takaichi, K.-i. Ueda, and A. A. Kaminskii, Optical Materials. 2007, 29, 1258-1262.

[91] D. Luo, J. Zhang, C. Xu, X. Qin, D. Tang, and J. Ma, Optical Materials. 2012, 34, 936-939.

[92] H. Yang, X. Qin, J. Zhang, J. Ma, D. Tang, S. Wang, and Q. Zhang, Optical Materials. 2012, 34, 940-943.

[93] X. Qin, H. Yang, G. Zhou, D. Luo, Y. Yang, J. Zhang, S. Wang, J. Ma, and D. Tang, Optical Materials. 2012, 34, 973-976.

[94] T. Zhao, H. Chen, D. Shen, Y. Wang, X. Yang, J. Zhang, H. Yang, and D. Tang, 
Optical Materials. 2013, 35, 712-714.

[95] A. Ikesue, Y. L. Aung, T. Taira, T. Kamimura, K. Yoshida, and G. L. Messing, Annual Review of Materials Research. 2006, 36, 397-429.

[96] H. Zhang, Q. Yang, S. Lu, and Z. Shi, Optical Materials. 2012, 34, 969-972.

[97] J. Zhang, L. An, M. Liu, S. Shimai, and S. Wang, Journal of the European Ceramic Society. 2009, 29, 305-309.

[98] A. Ikesue, K. Kamata, and K. Yoshida, Journal of the American Ceramic Society. 1996, 79, 359-364.

[99] F. Tang, Y. Cao, J. Huang, H. Liu, W. Guo, and W. Wang, Journal of the American Ceramic Society. 2012, 95, 56-69.

[100]N. Ter-Gabrielyan, L. Merkle, E. Kupp, G. Messing, and M. Dubinskii, Optics Letters. 2010, 35, 922-924.

[101] E. R. Kupp, G. L. Messing, J. M. Anderson, V. Gopalan, J. Q. Dumm, C. Kraisinger, N. Ter-Gabrielyan, L. D. Merkle, M. Dubinskii, and V. K. Simonaitis-Castillo, Journal of Materials Research. 2010, 25, 476-483.

[102] S. Lu, Q. Yang, Y. Wang, Y. Li, and D. Huang, Optical Materials. 2013, 35, 718721.

[103] J. Lu, M. Prabhu, J. Song, C. Li, J. Xu, K. Ueda, A. Kaminskii, H. Yagi, and T. Yanagitani, Applied Physics B. 2000, 71, 469-473.

[104] J. Lu, K.-i. Ueda, H. Yagi, T. Yanagitani, Y. Akiyama, and A. A. Kaminskii, Journal of Alloys and Compounds. 2002, 341, 220-225.

[105] J. Lu, H. Yagi, K. Takaichi, T. Uematsu, J.-F. Bisson, Y. Feng, A. Shirakawa, K.-I. Ueda, T. Yanagitani, and A. Kaminskii, Applied Physics B. 2004, 79, 25-28.

[106]A. Mandl, and D. E. Klimek, presented at Conference on Lasers and Electro-Optics. 2010.

[107] J. Sanghera, W. Kim, G. Villalobos, B. Shaw, C. Baker, J. Frantz, B. Sadowski, and I. Aggarwal, Optical Materials. 2013, 35, 693-699.

[108] J. Sanghera, W. Kim, G. Villalobos, B. Shaw, C. Baker, J. Frantz, B. Sadowski, and I. Aggarwal, Materials. 2012, 5, 258-277.

[109] J.-G. Li, T. Ikegami, J.-H. Lee, T. Mori, and Y. Yajima, Journal of the European Ceramic Society. 2000, 20, 2395-2405. 
[110] Y. L. Kopylov, V. Kravchenko, A. Komarov, Z. Lebedeva, and V. Shemet, Optical Materials. 2007, 29, 1236-1239.

[111] X. Li, H. Liu, J. Wang, H. Cui, X. Zhang, and F. Han, Materials Science and Engineering: A. 2004, 379, 347-350.

[112] Q. Chen, Y. Shi, S. Wang, J. Chen, and J. Shi, Journal of the European Ceramic Society. 2007, 27, 191-197.

[113] S. Hassanzadeh-Tabrizi, E. Taheri-Nassaj, and H. Sarpoolaky, Journal of Alloys and Compounds. 2008, 456, 282-285.

[114] R. Manalert, and M. Rahaman, Journal of Materials Science. 1996, 31, 3453-3458.

[115] K. Yamashita, K. Ramanujachary, and M. Greenblatt, Solid State Ionics. 1995, 81, 53-60.

[116] X. Li, H. Liu, J. Wang, H. Cui, F. Han, and R. Boughton, Journal of the American Ceramic Society. 2004, 87, 2288-2290.

[117] S. Bhattacharyya, and S. Ghatak, Ceramics International. 2009, 35, 29-34.

[118] M. Nyman, J. Caruso, M. J. Hampden, and T. T. Kodas, Journal of the American Ceramic Society. 1997, 80, 1231-1238.

[119] Y. Zhou, J. Lin, M. Yu, S. Han, S. Wang, and H. Zhang, Materials research bulletin. 2003, 38, 1289-1299.

[120] G. Xia, S. Zhou, J. Zhang, and J. Xu, Journal of Crystal Growth. 2005, 279, 357362.

[121]H. Kamiya, H. Suzuki, T. Ichikawa, Y. I. Cho, and M. Horio, Journal of the American Ceramic Society. 1998, 81, 173-179.

[122] I. Y. Prokhorov, and G. Y. Akimov, Journal of the European Ceramic Society. 1997, $17,129-131$.

[123]F. Tang, Y. Cao, J. Huang, W. Guo, H. Liu, Q. Huang, and W. Wang, Journal of the European Ceramic Society. 2012, 32, 3995-4002.

[124] X. Ba, J. Li, Y. Pan, Y. Zeng, H. Kou, W. Liu, J. Liu, L. Wu, and J. Guo, Journal of Alloys and Compounds. 2013, 577, 228-231.

[125] A. Kaminskii, V. Kravchenko, Y. L. Kopylov, S. Bagayev, V. Shemet, A. Komarov, F. Kallmeyer, and H. Eichler, Physica Status Solidi (a). 2007, 204, 2411-2415.

[126]K. A. Appiagyei, G. L. Messing, and J. Q. Dumm, Ceramics International. 2008, 34, 
1309-1313.

[127] Y. L. Kopylov, V. Kravchenko, S. Bagayev, V. Shemet, A. Komarov, O. Karban, and A. Kaminskii, Optical Materials. 2009, 31, 707-710.

[128] M. Edirisinghe, and J. Evans, International Journal of High Technology Ceramics. 1986, 2, 1-31.

[129] M. Edirisinghe, and J. Evans, International journal of high technology ceramics. 1986, 2, 249-278.

[130]T. Isobe, T. Tomita, Y. Kameshima, A. Nakajima, and K. Okada, Journal of the European Ceramic Society. 2006, 26, 957-960.

[131]D. J. Shanefield, Organic additives and ceramic processing: with applications in powder metallurgy, ink, and paint, Springer Science \& Business Media, 2013.

[132] R. M. German, Solar-Terrestrial Physics. 1996, 568.

[133]S.-J. L. Kang, Sintering: densification, grain growth and microstructure, Butterworth-Heinemann, 2004.

[134]C. B. Carter, and M. G. Norton, Ceramic materials: science and engineering, Springer Science \& Business Media, 2007.

[135] K. Johnson, K. Kendall, and A. Roberts, presented at Proceedings of the Royal Society of London A: Mathematical, Physical and Engineering Sciences 1971.

[136] R. L. Coble, Journal of Applied Physics. 1961, 32, 787-792.

[137] R. L. Coble, Journal of Applied Physics. 1961, 32, 793-799.

[138] A. Ikesue, Optical materials. 2002, 19, 183-187.

[139]H. Yang, J. Zhang, X. Qin, D. Luo, J. Ma, D. Tang, H. Chen, D. Shen, and Q. Zhang, Journal of the American Ceramic Society. 2012, 95, 52-55.

[140] K. Hamano, and S. Kanzaki, Journal of the Ceramic Society of Japan. 1977, 85, 225-230.

[141]R. Cook, M. Kochis, I. Reimanis, and H.-J. Kleebe, presented at Defense and Security. 2005.

[142] M. Bocanegra-Bernal, Journal of Materials Science. 2004, 39, 6399-6420.

[143] I. Tanaka, G. Pezzotti, T. Okamoto, Y. Miyamoto, and M. Koizumi, Journal of the American Ceramic Society. 1989, 72, 1656-1660.

[144] S. H. Lee, E. R. Kupp, A. J. Stevenson, J. M. Anderson, G. L. Messing, X. Li, E. C. 
Dickey, J. Q. Dumm, V. K. Simonaitis, and G. J. Quarles, Journal of the American Ceramic Society. 2009, 92, 1456-1463.

[145]M. Suárez, A. Fernández, J. Menéndez, M. Nygren, R. Torrecillas, and Z. Zhao, Journal of the European Ceramic Society. 2010, 30, 1489-1494.

[146]M. Tokita, presented at Proceeding of NEDO International Symposium on Functionally Graded Materials. 1999.

[147] R. Chaim, M. Kalina, and J. Z. Shen, Journal of the European Ceramic Society. 2007, 27, 3331-3337.

[148] L. An, A. Ito, and T. Goto, Journal of the American Ceramic Society. 2011, 94, 695698.

[149] M. Oghbaei, and O. Mirzaee, Journal of Alloys and Compounds. 2010, 494, 175189.

[150] M. A. Janney, C. L. Calhoun, and H. D. Kimrey, Journal of the American Ceramic Society. 1992, 75, 341-346.

[151] J. D. Katz, Annual Review of Materials Science. 1992, 22, 153-170.

[152] J. Cheng, D. Agrawal, Y. Zhang, and R. Roy, Materials Letters. 2002, 56, 587-592.

[153] M. Sekita, H. Haneda, T. Yanagitani, and S. Shirasaki, Journal of Applied Physics. 1990, 67, 453-458. 



\section{Chapter 3}

\section{Experimental Methodology}

This chapter presents the rationale and principle as justification to select ytterbium ion as the doping element and YAG garnet as host materials for laser ceramics. The fabrication approaches from powder processing to ceramic sintering are presented and discussed. The methods and techniques used to characterize phase, microstructure, optical and spectroscopic properties of the materials are described in a detailed way. The set-up of solid state laser systems used to characterize laser performances of the samples is described as well. 


\subsection{Rationale for selection of materials}

\subsubsection{Ytterbium ion as doping element}

Ytterbium $(\mathrm{Yb})$ is fourteenth element in the lanthanide series. Its most common oxidation state is $3+$, forming oxide compound of $\mathrm{Yb}_{2} \mathrm{O}_{3} . \mathrm{Yb}^{3+}$ ion has electron configuration of $4 \mathrm{f}^{13}$ structure. The energy levels of $\mathrm{Yb}^{3+}$ ion are shown in Figure 3.1 [1].

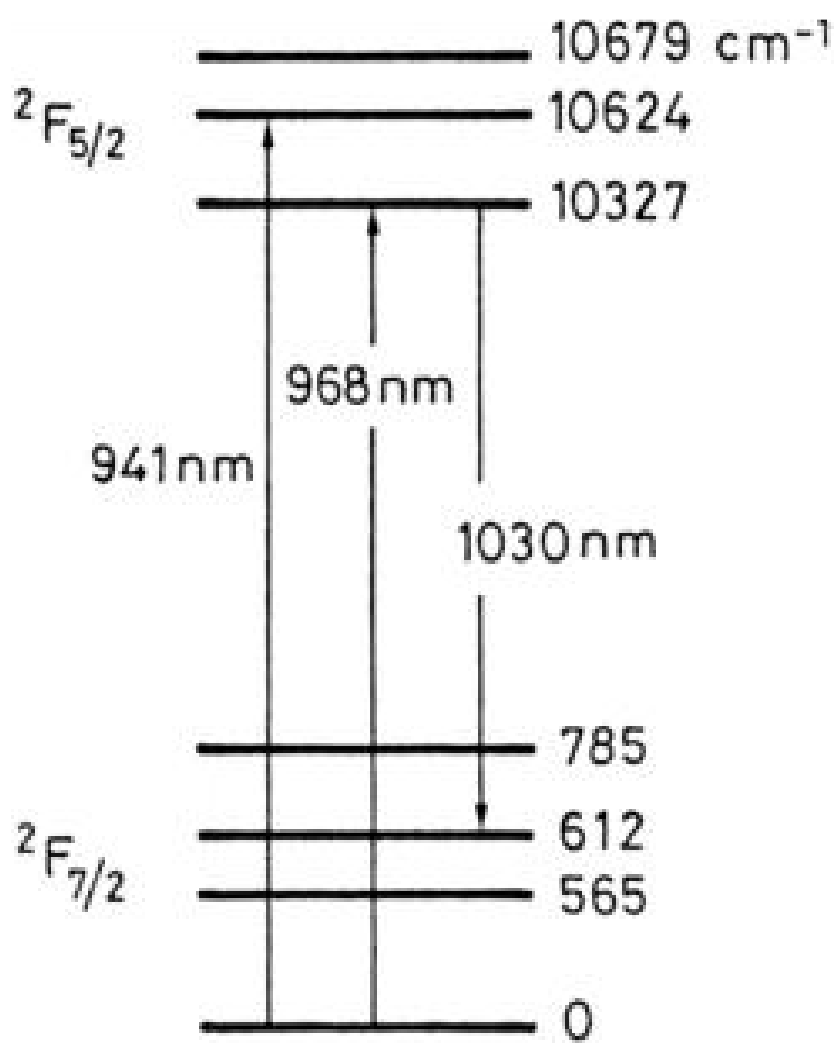

Figure 3.1 Energy levels of $\mathrm{Yb}^{3+}$ ions.

It has a very simple energy level structure, involving only two manifolds: one excited state manifold $\left({ }^{2} \mathrm{~F}_{5 / 2}\right)$ and one ground-state manifold $\left({ }^{2} \mathrm{~F}_{7 / 2}\right)$ [2]. The ytterbium-doped gain medium belongs to a quasi-three-level laser system [3]. In solid-state laser applications, it is commonly pumped by using InGaAs laser diodes at a broad band between $900 \mathrm{~nm}$ and $980 \mathrm{~nm}$, with lasing wavelength of $1030 \mathrm{~nm}$ [4-6]. There is also no up-conversion, no excited-state absorption or concentration quenching processes due to the simple 
electronic structure [7-9]. The quantum defect of $\mathrm{Yb}^{3+}$ ion is always very low, potentially allowing for very high power efficiencies of lasers. The gain bandwidth of the laser transitions is also fairly large, which allows for wide wavelength tuning ranges [10, 11] or generating ultra-short pulses in mode-locked lasers [12]. Its long upper-state lifetime is also beneficial for Q-switching laser operation [13].

Among the various laser medium, besides the very common neodymium $(\mathrm{Nd})$ doped material, the one comes next is the ytterbium doped material, such as ytterbium doped yttrium aluminum garnet ( $\mathrm{Yb}: \mathrm{YAG})$. The $\mathrm{Yb}$ doping concentration in YAG is usually in the range between 0.2 and 50 atomic percentages (at.\%). Higher doping of even 100 at.\% is also possible [14], while the crystal lattice structure will not be influenced too much with Yb doping level [15]. In Nd:YAG single crystal, the Nd doping is usually less than 1 at.\% due to the lower segregation coefficient [16]. It was difficult to grow good quality crystal with high $\mathrm{Nd}$ concentration using the traditional single crystal technology, whilst there's less of a problem for Yb:YAG. The other advantage in spectroscopic properties of $\mathrm{Yb}$ compared with $\mathrm{Nd}$ is that $\mathrm{Yb}$ possesses a longer florescent lifetime and broader emission bandwidth. This is especially beneficial for pump energy storage [17]. Yb doped materials also have a broader absorption bandwidth. The absorption band matches with the wavelength of commercially available laser diodes. This makes $\mathrm{Yb}$ materials very suitable for direct laser diode pumping. These advantages prove to be very useful for generating ultra short pulse in femtosecond lasers, which on the other hand is very difficult to achieve by the $\mathrm{Nd}$ doped material [17]. The appearance of $\mathrm{Yb}$ doped laser materials has played a crucial role in the development of the ultrashort-pulsed laser technology and has attracted great attentions of the laser scientists.

\subsubsection{YAG garnet ceramic laser host material}

Of various laser ceramics materials, yttrium aluminum garnet (YAG) is one which has been studied most intensively. The molecular formula of $\mathrm{YAG}$ is $\mathrm{Y}_{3} \mathrm{Al}_{5} \mathrm{O}_{12}$, it is a synthetic crystalline material of the garnet group, which belongs to one of the three 
phases in the yttrium-aluminium composite. The phase diagram of $\mathrm{Al}_{2} \mathrm{O}_{3}-\mathrm{Y}_{2} \mathrm{O}_{3}$ composite system is shown in the Figure 3.2 [18].

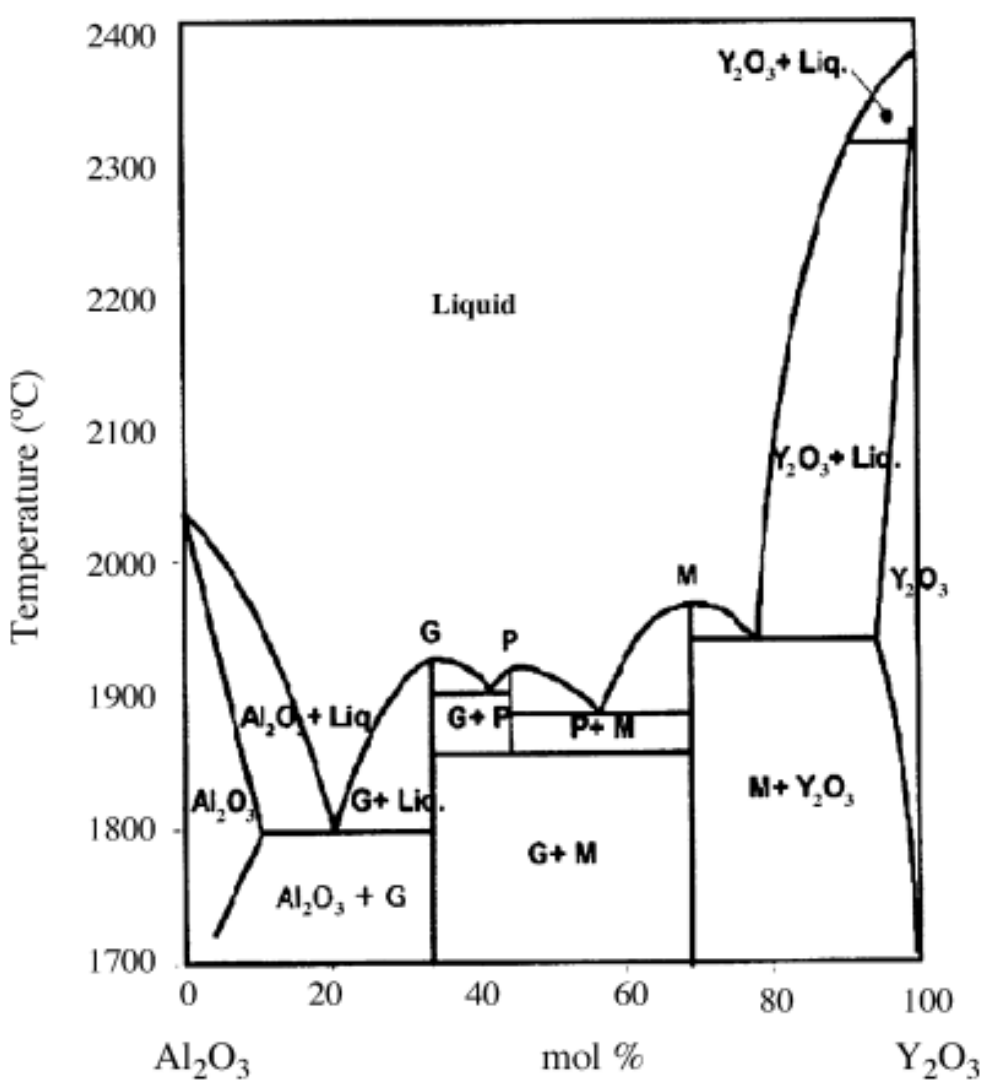

Figure 3.2 Phase diagram of $\mathrm{Al}_{2} \mathrm{O}_{3}-\mathrm{Y}_{2} \mathrm{O}_{3}$ composite system.

The formation of YAG phase and the intermediate phase YAM (yttrium aluminium monoclinic, $\mathrm{Y}_{4} \mathrm{~A}_{12} \mathrm{O}_{9}$ ) and $\mathrm{YAP}$ (yttrium aluminium perovskite $\mathrm{YAlO}_{3}$ ) of $\mathrm{Al}_{2} \mathrm{O}_{3}-\mathrm{Y}_{2} \mathrm{O}_{3}$ system are shown in following equations [19]:

$$
\begin{aligned}
& 2 \mathrm{Y}_{2} \mathrm{O}_{3}+\mathrm{Al}_{2} \mathrm{O}_{3} \rightarrow \text { YAM }\left(900-1100^{\circ} \mathrm{C}\right), \\
& \mathrm{YAM}+\mathrm{Al}_{2} \mathrm{O}_{3} \rightarrow 4 \mathrm{YAP}\left(1100-1250^{\circ} \mathrm{C}\right), \\
& 3 \mathrm{YAP}+\mathrm{Al}_{2} \mathrm{O}_{3} \rightarrow \mathrm{YAG}\left(1400-1600^{\circ} \mathrm{C}\right) .
\end{aligned}
$$

YAG crystal has a cubic lattice structure with lattice constant of $12.000 \pm 0.002 \AA$ [20]. 
There are eight $\mathrm{Y}_{3} \mathrm{Al}_{5} \mathrm{O}_{12}$ molecules in one cell. $\mathrm{Y}^{3+}$ ions locate at centre of the dodecahedrons, $\mathrm{Al}^{3+}$ ions are at center of the octahedron and tetrahedrons, surrounded by $\mathrm{O}^{2-}$ ions [21]. Figure 3.3 shows crystal structure of YAG.

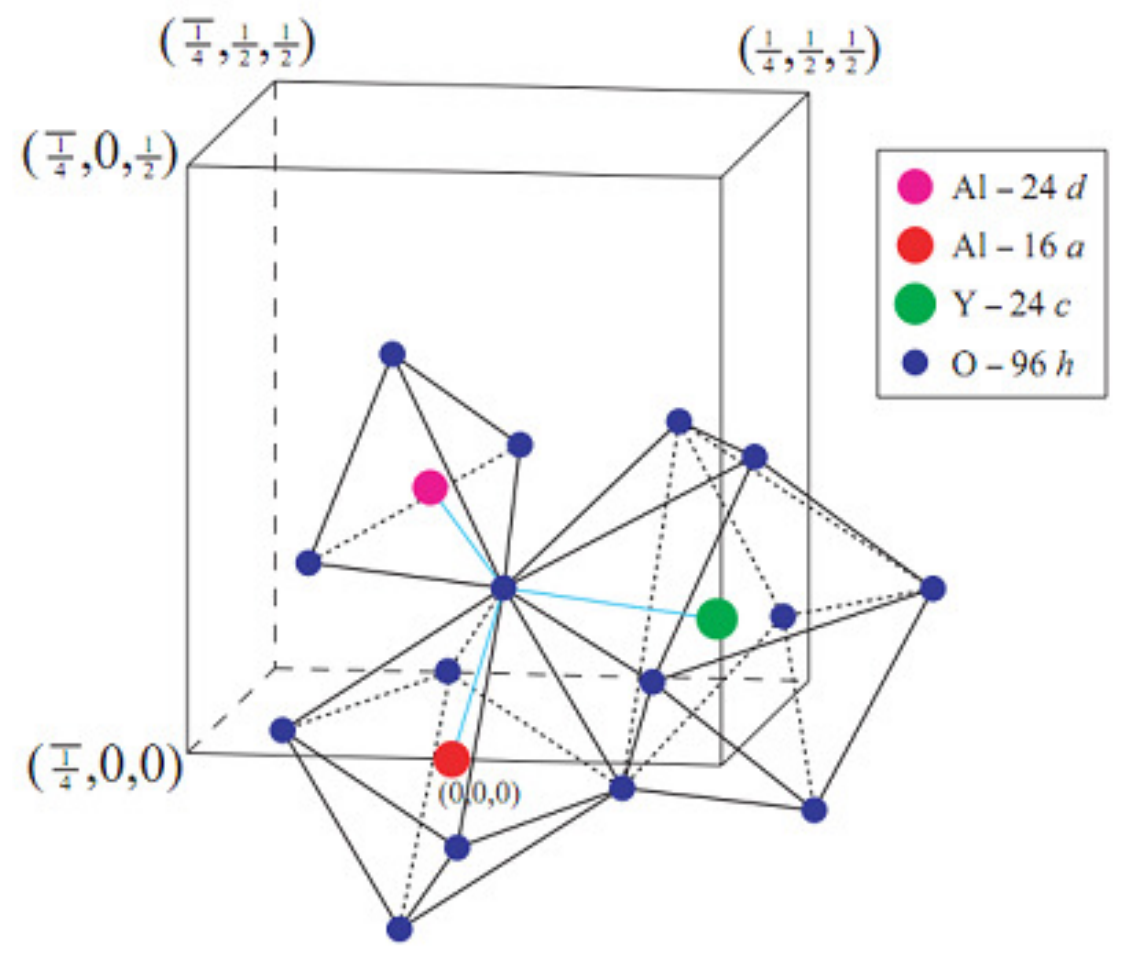

Figure 3.3 Crystal structure of YAG.

YAG is most commonly used in solid-state lasers as a host material. Some properties of YAG are listed in Table 3.1 [22]. The cubic crystal structure makes YAG optically isotropic. The high theoretical transmittance also favors YAG to be suitable as a laser material. Rare earth elements $\left(\mathrm{Re}^{3+}\right)$, such as neodymium $\left(\mathrm{Nd}^{3+}\right)$ and ytterbium $\left(\mathrm{Yb}^{3+}\right)$ can be doped into YAG, to partially or fully replace $\mathrm{Y}^{3+}$ ion positions as active laser ions, yielding rare-earth ion doped YAG (Re:YAG) lasers. Various YAG based materials have been studied over the years as a novel laser gain media for solid state laser applications [22-25]. With the development of ceramics processing technology, YAG transparent laser ceramics have also attracted many attentions in recent years [26-30]. 
Table 3.1 Properties of YAG crystal.

\begin{tabular}{l|l}
\hline Chemical formula & $\mathrm{Y}_{3} \mathrm{Al}_{5} \mathrm{O}_{12}$ \\
\hline Molecular weight & 593.62 \\
\hline Density & $4.55 \mathrm{~g} / \mathrm{cm}^{3}$ \\
\hline Crystal system & cubic \\
\hline Refractive index & $1.81(1.06 \mu \mathrm{m})$ \\
\hline Theoretical transmittance & $84.8 \%$ \\
\hline Mohs scale hardness & $8 \sim 8.5$ \\
\hline Melting point & $1950^{\circ} \mathrm{C}$ \\
\hline Thermal conductivity & $\sim 10 \mathrm{~W} / \mathrm{m} / \mathrm{K}$ \\
\hline
\end{tabular}

\subsection{Laser ceramics fabrication method}

\subsubsection{Ball milling processing}

Ball milling is used for the purpose of mixing the powders into homogeneous mixture. High energy planetary milling machine are used [31]. The schematic drawing for the milling machine is shown in following Figure 3.4.
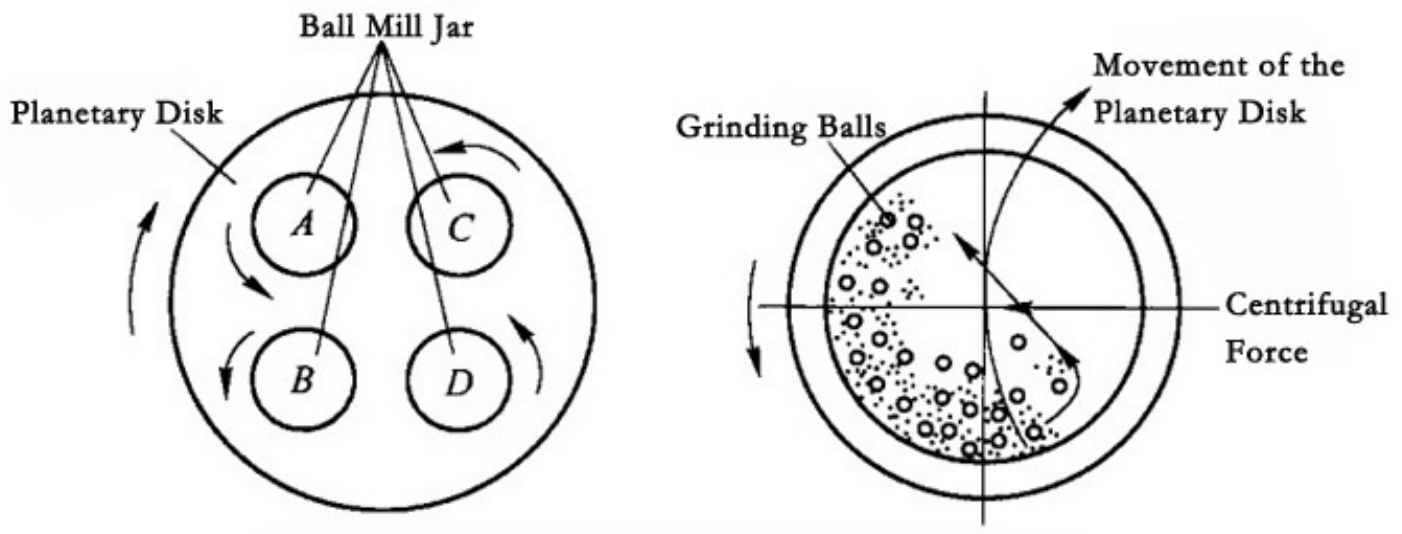

Figure 3.4 Schematic drawing of high energy planetary ball milling system with (left) layout of planetary disk and (right) horizontal view of milling jar. 
The milling jars are located eccentrically on the planetary disk and rotate to the opposite direction of the disk. The balls in the milling jars will then be in a complex rotational movement. High momentum energies will be released as the frictional force and impact force between the milling balls, jars and the powders. The overall ball milling effect is to break the powder agglomerations and reduce the powder size, as well as the homogeneous mixing. The materials for the balls and jars are usually of high purity with minimal wear out. This is to minimize the debris fallen from the jar and ball themselves and cause contamination the milling material. Commercially available raw powders were used as raw materials. The reason is that they can be obtained in relative large amount, and stable properties between various batches if they come from the same source. Thus repetitiveness of the experiments can be assured. The powder purity is typically $>99.99 \%$. Calcination process is often carried out before milling process. The calcinations temperature is around $\sim 500^{\circ} \mathrm{C}$ to $800^{\circ} \mathrm{C}$ in muffle furnace in air environment. The purpose is to remove of moistures vapor and volatile gases if presented

\subsubsection{Dry press and cold isostatic pressing process}

Dry press and cold isostatic pressing (CIP) method were used to form loose powder into shaped green-body. Figure.3.5 shows the schematic diagram of the single action uniaxial press forming and cold isostatic press forming. Loose powders are firstly poured into the metal die and relatively low pressure ( $20 \mathrm{MPa})$ is applied from top and bottom in a single axis to squeeze the powders to form a preliminary shape of green body. The green body is then ejected out from the die with care since it is relatively fragile at this stage. After wrapping with water proved elastic encapsulation such as a rubber mold, the green body will then be placed into the CIP machine chamber filled with water or oil. A much higher pressure ( 200MPa) is applied and the water liquid will give isostatic pressing to the green body. This can gives a better uniformity in packing density. The method is simple and straight and has the advantage of good repeatability. 


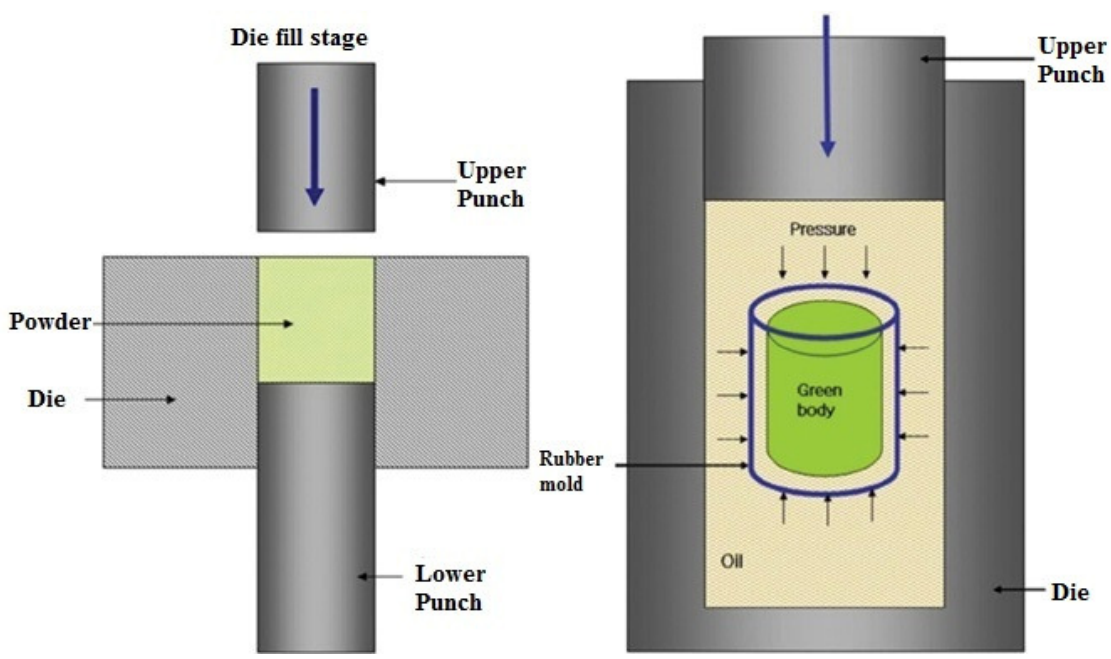

Figure 3.5 Schematic diagrams of the uniaxial mold and cold isostatic pressing.

\subsubsection{Vacuum sintering process}

High temperature vacuum sintering furnace was used for samples densification. The photo of sintering furnace is shown in Figure 3.6. The maximum size of a sample could be sintered is limited by the furnace chamber size. The furnace is able to reach a temperature of $2000^{\circ} \mathrm{C}$ and can maintain a vacuum level of higher than $\sim 10^{-4} \mathrm{~Pa}$.

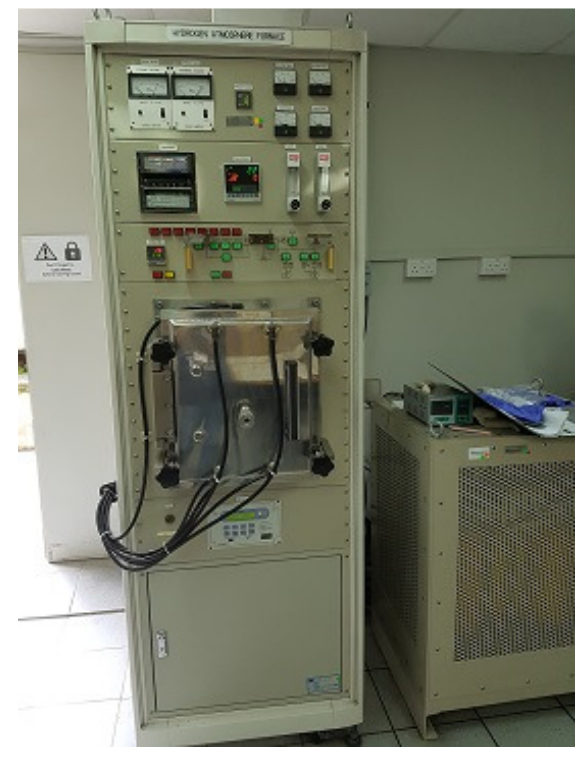

Figure 3.6 Photograph of a high temperature vacuum sintering furnace. 


\subsection{Characterization method}

\subsubsection{XRD analysis}

Material phase and crystal structures of fabricated samples are characterized by using Xray diffraction (XRD Bruker D8, Cu Ka radiation). The schematic drawing of Bragg diffraction is shown in Figure 3.7. When the X-ray passed through sample and scattered by the crystal lattices, the interference patterns are created in the reflected waves. Constructive interference occurs when the diffraction angle $\theta$ match the Bragg's law [32]: $N \lambda=2 d \theta$

where $\mathrm{N}$ is the wavelength number, $\lambda$ is the wavelength of the $\mathrm{x}$-ray, $\mathrm{d}$ is the lattice constant of the crystal. A peak in the diffraction pattern is then observed.

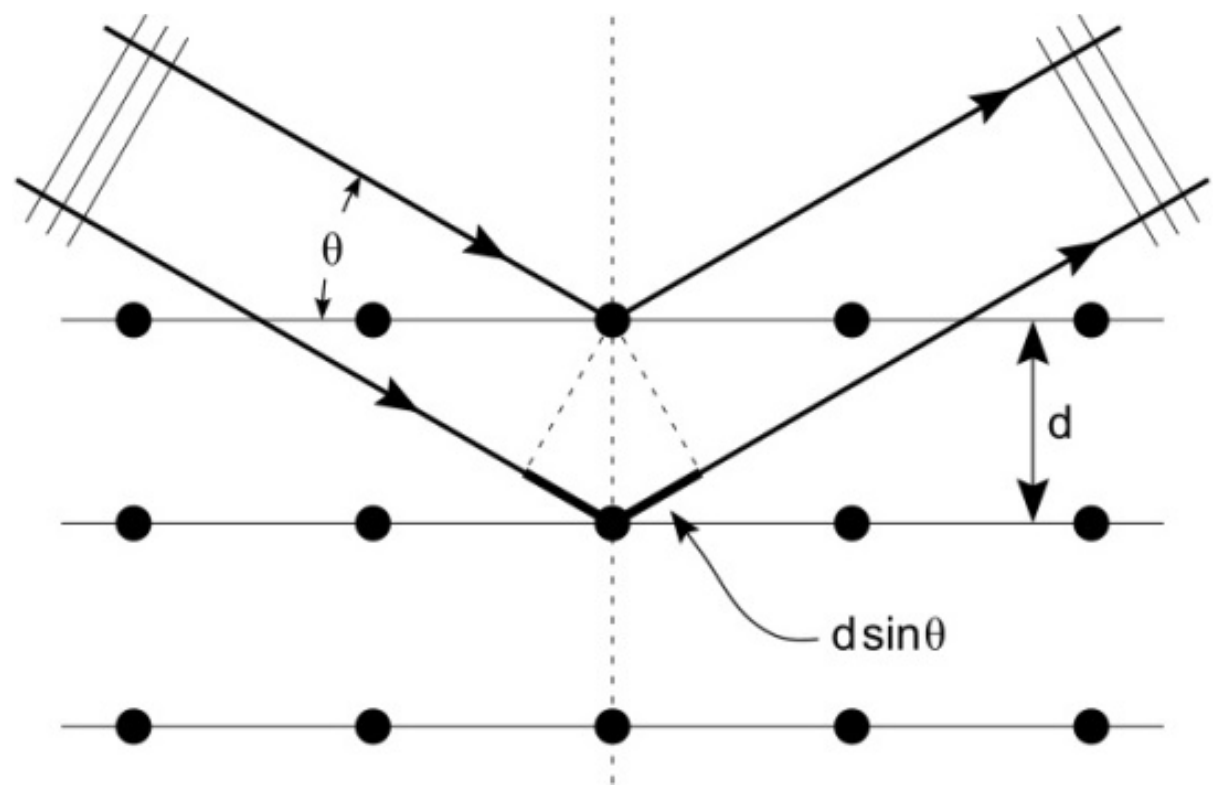

Figure 3.7 Schematic drawing of Bragg diffraction.

The samples were scanned with $2 \theta$ range from $10-100^{\circ}$. The measured samples' XRD patterns were recorded compared with standard database for analysis. XRD is an accurate method for identifying sample's crystal phase and structure. 


\subsubsection{SEM analysis}

Scanning Electron Microscope (SEM) JEOL 6360 were used to characterize samples' morphology and microstructure. It produces the images of the sample by scanning it with a focused beam of electrons. The electrons interact with atoms in the sample, producing various signals that can be detected and that contain information about the sample's surface topography and composition. Figure 3.8 shows the typical schematic of an SEM. The resolution of SEM can achieve as high as $1 \mathrm{~nm}$. It is determined by in the Abbe's equation [33]:

$D=\frac{0.612 \lambda}{N A}$

where $\lambda$ is the wavelength of electron, $\mathrm{D}$ is the resolution and NA is the numerical aperture of the SEM system.

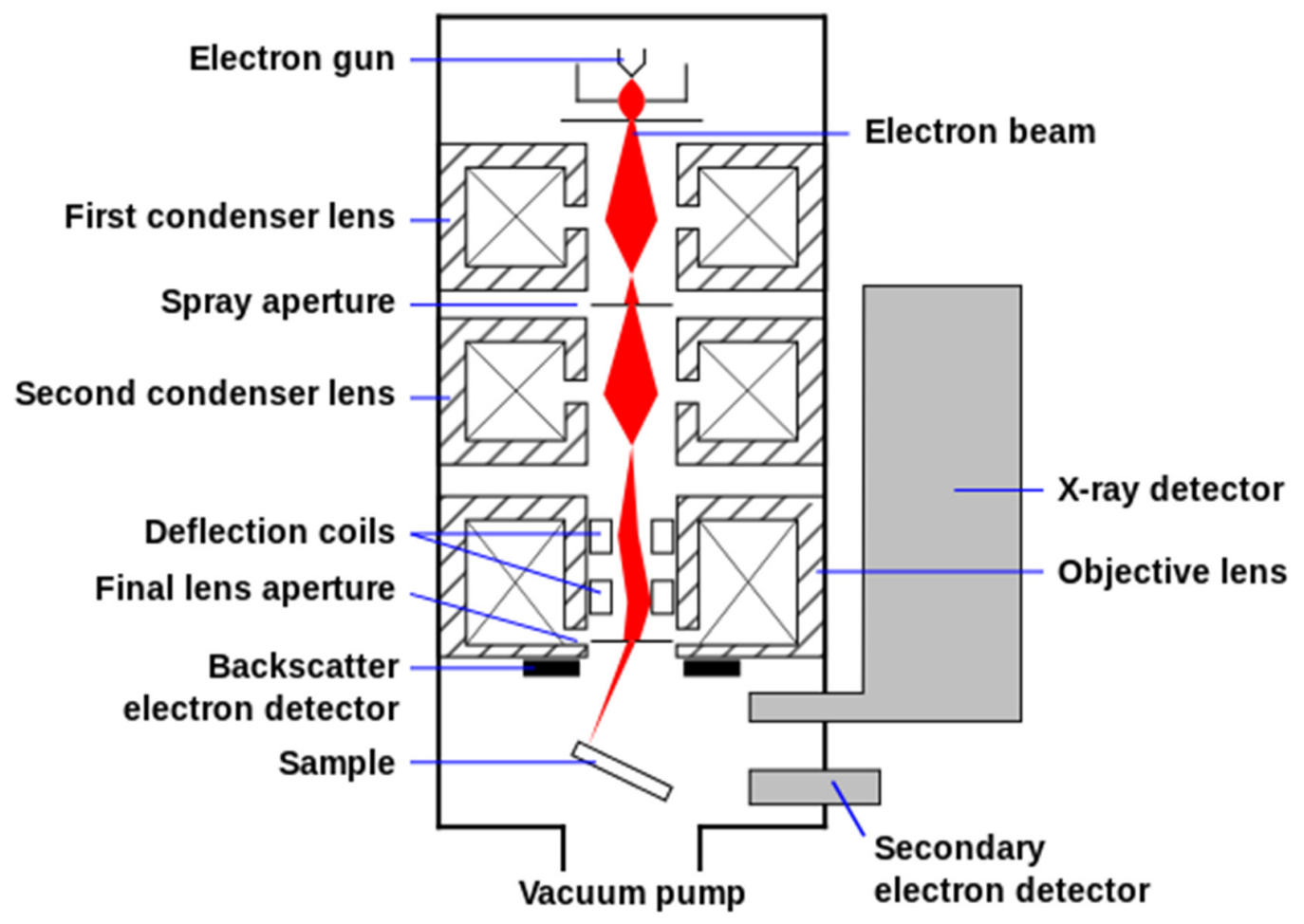

Figure 3.8 Schematic drawing of SEM equipment.

For powder samples, the particle size, distribution, level of agglomeration will reflect the 
powders properties. For bulk samples, the samples' grains shape, grain size, grain distribution and pores size/distribution will also be a good reflection and linked to the samples' optical/mechanical quality. SEM is a very useful method for topography and morphology characterization.

\subsubsection{Spectrophotometer analysis}

Spectrophotometer is used to measure the room temperature optical in-line transmittance of sintered sample. UV-VIS-NIR Spectrophotometer is used for characterizing the optical properties of the measured sample like transmittance and reflectance. The principle for measuring transmittance or reflectance is based on the calculated percentage of the transmitted or reflected light intensity with reference to the incident light intensity. The basic configuration of a spectrophotometer is shown in Figure 3.9.

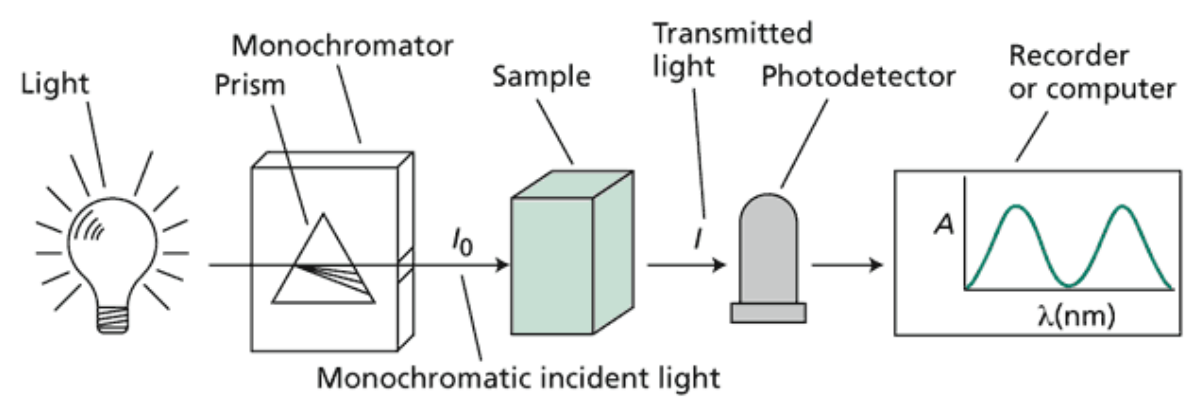

Figure 3.9 Schematic drawing of configuration of a spectrophotometer.

The light source generates a range of light wavelength. Typical light sources are halogen lamp. Since the generated light combines a lot of different wavelengths, monochrometer is needed to split them into separate components for single wavelength characterization. A prism or a diffraction grating can work as the monochrometer to split light to different exit slits. Samples are placed inside the holder in the sample compartment with the side of the sample perpendicular to the incident light beam to avoid reflection in other angles. Finally, photodetectors are placed behind the samples to measure the intensity of the remaining light beam. Light intensity attenuates while passing through the bulk samples body. So the transmittance value $\mathrm{T}$ is typically less than $100 \%$. Spectrophotometer 
method can gives sample's optical transmittance quantitatively, which is a good reflection of sample's optical quality.

\subsubsection{Spectrofluorometer analysis}

A spectrofluorometer is an instrument used to measure the fluorescent properties of the samples. A certain excitation wavelength from light source is selected to excite the sample. The wavelength is typically close to sample's strongest absorption wavelength for maximizing its absorption efficiency. The light beam is usually passed through a monochromator and the shine onto the sample. The emission light from the sample is collected typically at 90 degrees to the exciting light, to avoid the interference directly from the light source. The emission is also either passed through a filter or a monochromator before being detected by a photodetector or charge-coupled device (CCD) detector. The emission intensity versus wavelength of the sample is recorded and plotted, which gives the emission spectra of the samples. The schematic drawing of configuration of a spectrofluorometer is shown in Figure 3.10.

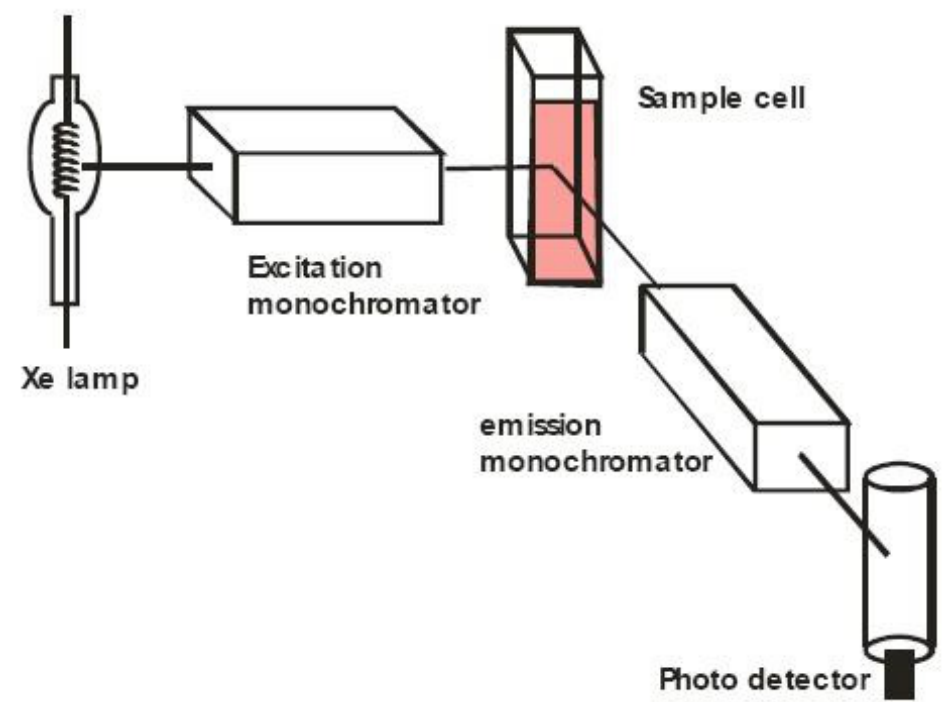

Figure 3.10 Schematic drawing of configuration of a spectrofluorometer. 


\subsubsection{Solid state laser system}

Figure 3.11 shows the schematic drawing of a simple tow mirror cavity solid state laser system. It shows the optical resonator, laser gain medium and pump source. In experiment, the pump source is replaced by a fiber coupled laser diode, with a focusing mirror to direct the laser beam on to the laser ceramics. The advantage of direct laser diode pumping is that we can choose respective laser diode source to match with the specific sample's absorption, thus increase absorption efficiency. The ceramics being tested are polished and AR coated on both pumping and lasing facets, to reduce surface reflection loss. Output laser power is measured by power meter. The two mirror solid laser system is used to test the laser performances of the fabricated ceramic samples. Modification and design of the laser cavity will depend on the practical laser experiment on the specific situation.

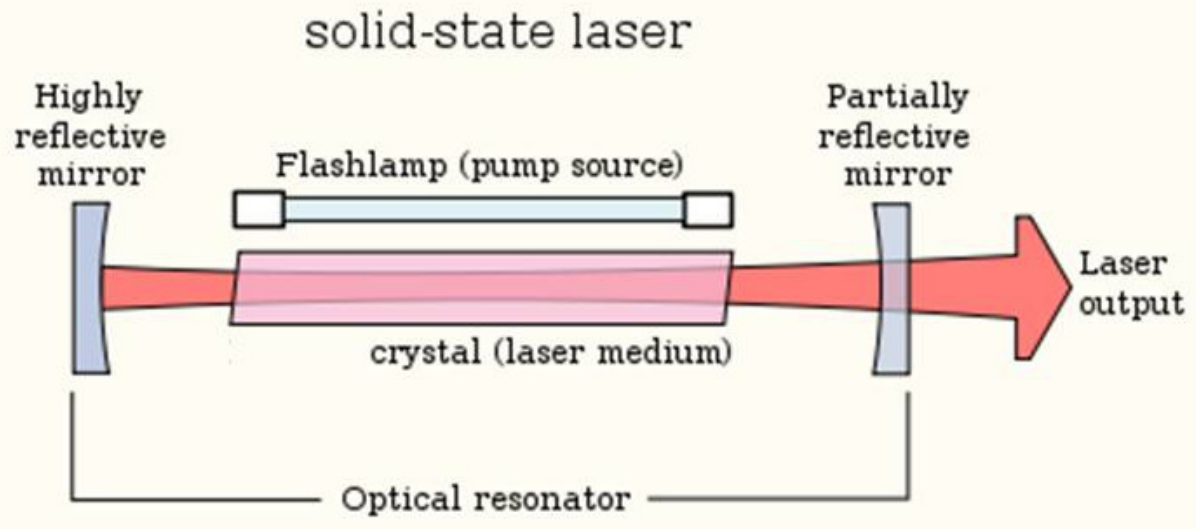

Figure 3.11 Schematic drawing of a typical solid state laser system.

\subsection{Overview of methodologies}

In this chapter, we first discussed the rational for selection of ytterbium ion as the active element and YAG garnet as the host material for laser ceramics. The ytterbium ion has the advantage of high quantum efficiency, which gives the possibility of obtaining high power and high efficiency laser performance. The fabrication process from powder process including ball milling, to green body shaping and vacuum sintering method were 
then introduced in details. The most cost effective method for repeatable sample fabrication was established, which is a demonstration of the in-house ceramic fabrication capability of our group. SEM and XRD techniques were used to characterize the phase, morphology and micro-structure of the powder and ceramic samples. The optical and spectroscopic properties of the samples were characterized with spectrophotometer and spectrofluorometer. Finally, the solid-laser system was presented for laser performances of the fabricated ceramic samples. This chapter provides theoretical understanding and a clear view of the techniques and approaches for the fabrication and characterization of transparent laser ceramics as well as the solid state laser system using obtained ceramic samples.

\section{References}

[1] O. Svelto, and D. C. Hanna, Principles of lasers, Springer, 1976.

[2] D. Wood, The Journal of Chemical Physics. 1963, 39, 1671-1673.

[3] R. J. Beach, Optics Communications. 1996, 123, 385-393.

[4] P. Lacovara, H. Choi, C. Wang, R. Aggarwal, and T. Fan, Optics Letters. 1991, 16, 1089-1091.

[5] T. Y. Fan, S. Klunk, and G. Henein, Optics Letters. 1993, 18, 423-425.

[6] H. W. Bruesselbach, D. S. Sumida, R. A. Reeder, and R. W. Byren, IEEE Journal of Selected Topics in Quantum Electronics. 1997, 3, 105-116.

[7] P. Yang, P. Deng, and Z. Yin, Journal of Luminescence. 2002, 97, 51-54.

[8] J. Dong, K. Ueda, H. Yagi, A. Kaminskii, and Z. Cai, Laser Physics Letters. 2008, 6, 282.

[9] R. Paschotta, J. Nilsson, P. Barber, J. Caplen, A. C. Tropper, and D. C. Hanna, Optics Communications. 1997, 136, 375-378.

[10] S. Nakamura, H. Yoshioka, Y. Matsubara, T. Ogawa, and S. Wada, Optics Communications. 2008, 281, 4411-4414.

[11] S. Nakamura, H. Yoshioka, T. Ogawa, and S. Wada, presented at Advanced SolidState Photonics. 2009. 
[12] E. Innerhofer, T. Südmeyer, F. Brunner, R. Häring, A. Aschwanden, R. Paschotta, C. Hönninger, M. Kumkar, and U. Keller, Optics Letters. 2003, 28, 367-369.

[13] J. Dong, A. Shirakawa, K.-i. Ueda, H. Yagi, T. Yanagitani, and A. A. Kaminskii, Applied Physics Letters. 2007, 90, 191106.

[14] F. D. Patel, E. C. Honea, J. Speth, S. A. Payne, R. Hutcheson, and R. Equall, IEEE Journal of Quantum Electronics. 2001, 37, 135-144.

[15] X. Xu, Z. Zhao, J. Xu, and P. Deng, Journal of Crystal Growth. 2003, 255, 338-341.

[16] K.-i. Shiroki, and Y. Kuwano, Nippon Kagaku Kaishi. 1978, 1978, 940-944.

[17] W. F. Krupke, IEEE Journal of Selected Topics in Quantum Electronics. 2000, 6, 1287-1296.

[18] S. Kochawattana, A. Stevenson, S.-H. Lee, M. Ramirez, V. Gopalan, J. Dumm, V. K. Castillo, G. J. Quarles, and G. L. Messing, Journal of the European Ceramic Society. 2008, 28, 1527-1534.

[19] J. Abell, I. Harris, B. Cockayne, and B. Lent, Journal of Materials Science. 1974, 9, 527-537.

[20] M. M. Kuklja, Journal of Physics: Condensed Matter. 2000, 12, 2953.

[21] Y.-N. Xu, and W. Ching, Physical Review B. 1999, 59, 10530.

[22] J. Geusic, H. Marcos, and L. Van Uitert, Applied Physics Letters. 1964, 4, 182-184.

[23] R. Stoneman, and L. Esterowitz, Optics Letters. 1990, 15, 486-488.

[24] T. Y. Fan, G. Huber, R. L. Byer, and P. Mitzscherlich, IEEE Journal of quantum Electronics. 1988, 24, 924-933.

[25] Y. E. Young, S. D. Setzler, K. J. Snell, P. A. Budni, T. M. Pollak, and E. Chicklis, Optics letters. 2004, 29, 1075-1077.

[26] A. Ikesue, I. Furusato, and K. Kamata, Journal of the American Ceramic Society. 1995, 78, 225-228.

[27] Y. Wu, J. Li, Y. Pan, J. Guo, B. Jiang, Y. Xu, and J. Xu, Journal of the American Ceramic Society. 2007, 90, 3334-3337.

[28] W. X. Zhang, Y. B. Pan, J. Zhou, W. B. Liu, J. Li, B. X. Jiang, X. J. Cheng, and J. Q. Xu, Journal of the American Ceramic Society. 2009, 92, 2434-2437.

[29] H. Yang, J. Zhang, X. Qin, D. Luo, J. Ma, D. Tang, H. Chen, D. Shen, and Q. Zhang, Journal of the American Ceramic Society. 2012, 95, 52-55. 
[30] X. Qin, H. Yang, G. Zhou, D. Luo, Y. Yang, J. Zhang, S. Wang, J. Ma, and and D. Tang, Optical Materials. 2012, 34, 973-976.

[31] H. Mio, J. Kano, F. Saito, and K. Kaneko, Materials Science and Engineering: A. 2002, 332, 75-80.

[32] D. M. Moore, and R. C. Reynolds, X-ray Diffraction and the Identification and Analysis of Clay Minerals, Oxford University press, 1989.

[33] W. Zhou, and Z. L. Wang, Scanning microscopy for nanotechnology: techniques and applications, Springer science \& business media, 2007. 


\section{Chapter 4*}

\section{Development of Yb:YAG Transparent Laser Ceramics}

Ytterbium doped YAG laser ceramics have many applications as $1 \mu \mathrm{m}$ solid state laser. The key is to obtain high quality laser ceramics with high optical transparency. In this chapter, the fabrication technological for $Y b: Y A G$ ceramics is presented. High quality $Y b: Y A G$ laser ceramics with different $\mathrm{Yb}$ doping concentration is obtained by using solid-state reaction and vacuum sintering method. The spectroscopic properties of samples are investigated. Solid state laser performances of samples are tested.

*This section published substantially as: Luo Dewei et al., "Fabrication and laser properties of transparent Yb:YAG ceramics." Optical Materials, 2012, 34, 936-939. and Luo De Wei et al., "Fabrication and Spectroscopic Properties of Transparent Yb:YAG Laser Ceramics." Solid State Phenomena, 2012, 185, 44-47. 


\subsection{Introduction}

Ytterbium doped yttrium aluminium garnet (Yb:YAG) laser material have attracted great attention due to the advantages such as high quantum efficiency, long fluorescence lifetime, broad emission spectrum $[1,2]$. Yb:YAG laser material belongs to the quasithree-level laser gain media [3,4]. It has a simple energy level structure of only two manifolds, the excited state manifold ${ }^{2} \mathrm{~F}_{5 / 2}$ and the ground state manifold ${ }^{2} \mathrm{~F}_{7 / 2}$ [5]. This simple energy level excludes excited-state absorption, as well as the concentration quenching effect in Yb:YAG [6]. The theoretical ytterbium doping concentration in Yb:YAG could be quite high, even can reach $100 \%$ doping concentration to from the composition of YbAG garnet [7, 8]. The broad absorption band of $\mathrm{Yb}^{3+}$ also matches with the wavelength of InGaAs laser diode, so it makes Yb:YAG very suitable for direct laser diode pumping [9]. These advantages make Yb:YAG a good candidate material for high power thin disk laser and ultra-short pulse laser applications [10-20].

With the development of ceramic processing technology, Yb:YAG laser material in form of polycrystalline ceramics have also been studied in recent years [21-23]. Takaichi et al. demonstrated a diode end-pumped Yb:YAG ceramics laser for the first time in 2003 [24]. Dong et al. demonstrated a Yb:YAG ceramics microchip laser with a slope efficiency as high as $79 \%$ in 2006 [25]. Excellent tunable Yb:YAG ceramics laser was also reported by Nakamura et al. in 2008 [26]. The optical properties of erbium and ytterbium co-doped YAG ceramics was studied by Zhou et al. in 2011 [27]. A composite Yb:YAG ceramics laser was reported by Tang et al. in 2012 [28]. The cryogenic properties of Yb:YAG ceramics has also been explored [29-31]. Yet, there's still not many detailed reports on the fabrication of transparent Yb:YAG laser ceramics. In this chapter, we employed the solid-state reaction and vacuum sintering method for high quality Yb:YAG ceramics fabrication. The raw powder materials were processed through ball milling, sieving and shaping process. Sintering additives were added to help densification and improve optical quality. High temperature vacuum sintering furnace was utilized for sample sintering process. The optical and spectroscopic properties of in-house fabricated Yb:YAG 
ceramics samples were measured and analyzed. Solid state laser system was also set up for testing the sample laser performances.

\subsection{Experimental methods}

\subsubsection{Fabrication process of Yb:YAG laser ceramics}

The Yb:YAG laser ceramics were fabricated by using the solid-state reaction and vacuum sintering method. The flowchart of the experimental fabrication process is shown in Figure 4.1. Raw materials used were high-purity (>99.99\%) $\mathrm{Al}_{2} \mathrm{O}_{3}, \mathrm{Y}_{2} \mathrm{O}_{3}$ and $\mathrm{Yb}_{2} \mathrm{O}_{3}$ powders. The powders were kept in an oven at $\sim 150^{\circ} \mathrm{C}$ to avoid absorbing moisture in air. Each respective powder were weighed precisely according to chemical compositions of $\mathrm{Y}_{3} \mathrm{Al}_{5} \mathrm{O}_{12}$ with different $\mathrm{Yb}^{3+}$ doping concentrations, and then mixed with $99.99 \%$ analytical ethanol through ball milling process.

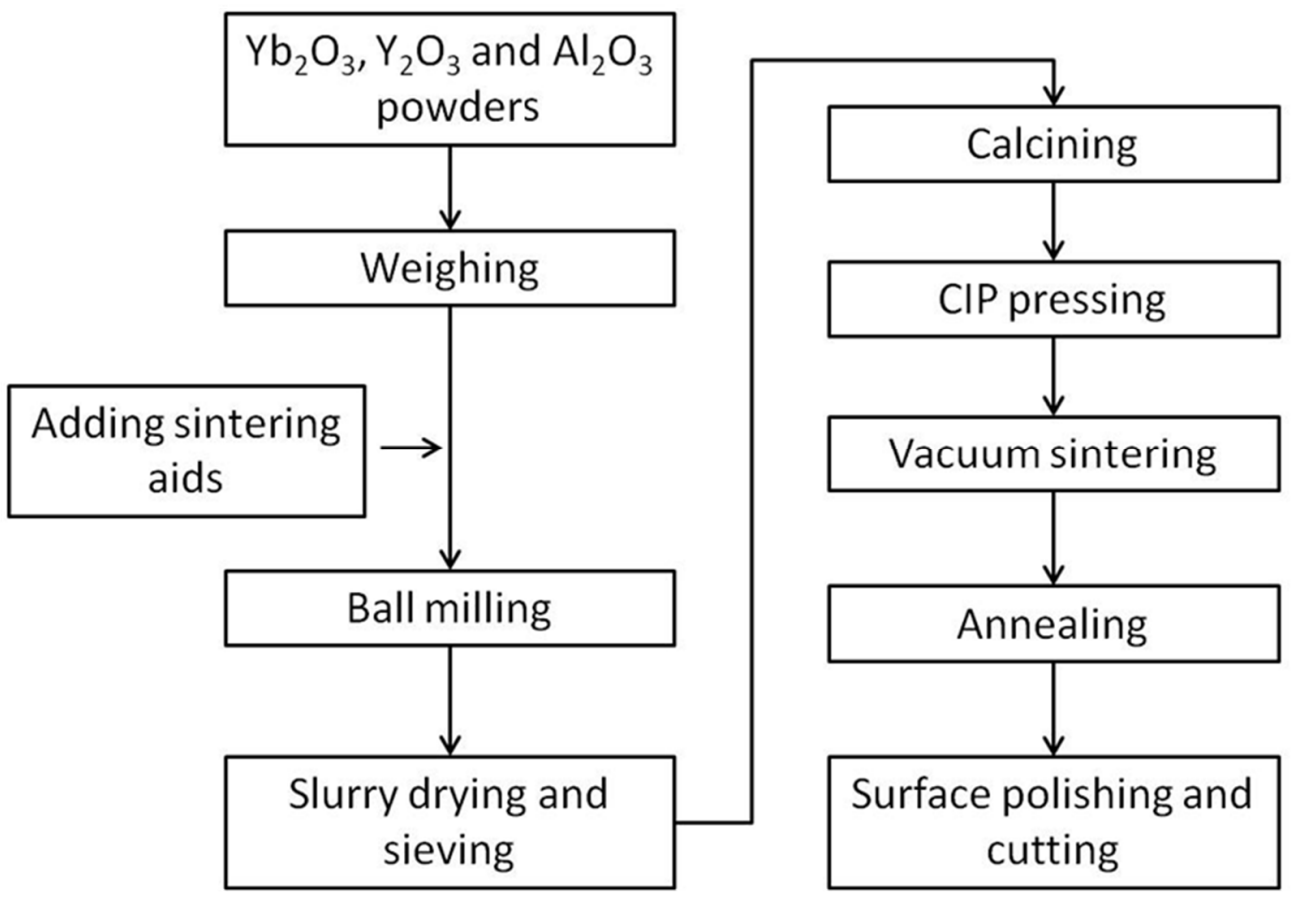

Figure 4.1 Fabrication flow chart of Yb:YAG laser ceramics. 
The mixed slurries were then placed in an aluminum jar and ball milled by using a high energy planetary milling machine at $180 \mathrm{rpm}$ for $15 \mathrm{~h}$. High purity alumina balls were used to avoid introducing foreign ingredient. Sintering additives were added during raw powder mixing process to improve densification [32, 33]. Tetraethyl orthosilicate (TEOS, Sigma-Aldrich, 99.99\%) was added to introduce $\mathrm{SiO}_{2}$ as sintering aids. The TEOS amount was $0.5 \mathrm{wt} . \%$, corresponding to a $0.14 \mathrm{wt} . \%$ of $\mathrm{SiO}_{2}$. The composition parameters of $\mathrm{Yb}$ :YAG ceramics with different doping concentration are summarized in Table 4.1.

Table 4.1 Composition parameters of Yb:YAG ceramics with different doping concentration.

\begin{tabular}{|c|c|c|c|c|c|c|}
\hline & \multicolumn{6}{|c|}{ Parameters } \\
\hline $\begin{array}{c}\mathrm{Yb}^{3+} \text { doping } \\
\text { concentration }\end{array}$ & 1.0 at.\% & 5.0 at. $\%$ & 8.0 at.\% & 10.0 at.\% & 15.0 at.\% & 20.0 at. $\%$ \\
\hline $\mathrm{Yb}_{2} \mathrm{O}_{3}(\mathrm{~g})$ & 0.4958 & 2.4377 & 3.8522 & 4.7759 & 7.0207 & 9.1775 \\
\hline $\mathrm{Y}_{2} \mathrm{O}_{3}(\mathrm{~g})$ & 28.1249 & 26.5390 & 25.3839 & 24.6296 & 22.7964 & 21.0350 \\
\hline $\mathrm{Al}_{2} \mathrm{O}_{3}(\mathrm{~g})$ & 21.3793 & 21.0232 & 20.7639 & 20.5945 & 20.1829 & 19.7875 \\
\hline $\mathrm{TEOS}(\mathrm{ml})$ & 0.26 & 0.26 & 0.26 & 0.26 & 0.26 & 0.26 \\
\hline
\end{tabular}

The milled mixtures were dried at $80^{\circ} \mathrm{C}$ for $24 \mathrm{~h}$ in oven and then manually sieved through 200-mesh screen to obtain fine particle. Then the powders were then gone through calcinations at $800^{\circ} \mathrm{C}$ for $3 \mathrm{~h}$ to remove organic components. The powders were then dry pressed with a stainless steel die at $15 \mathrm{MPa}$ into pellets. The pellets were further cold isostatically pressed (CIP) at $200 \mathrm{MPa}$ to form the green bodies. The green bodies were then placed in the tungsten crucible and sintered in a high temperature vacuum sintering furnace at $1780^{\circ} \mathrm{C}$ with the vacuum level higher than $10^{-4} \mathrm{~Pa}$ for densification. The sintered pellets were then annealed in air at $1400^{\circ} \mathrm{C}$ for up to $30 \mathrm{~h}$ to release internal stress and eliminate oxygen vacancies. All sintered samples were firstly grinded by using a horizontal grinding machine (Model: EHG-150, Engis, Japan) and then fine polished by using a precision lapping machine (Model: EJW400-IN-D, Engis, Japan). After polishing, the pellets had parallel and mirror-like surfaces. 

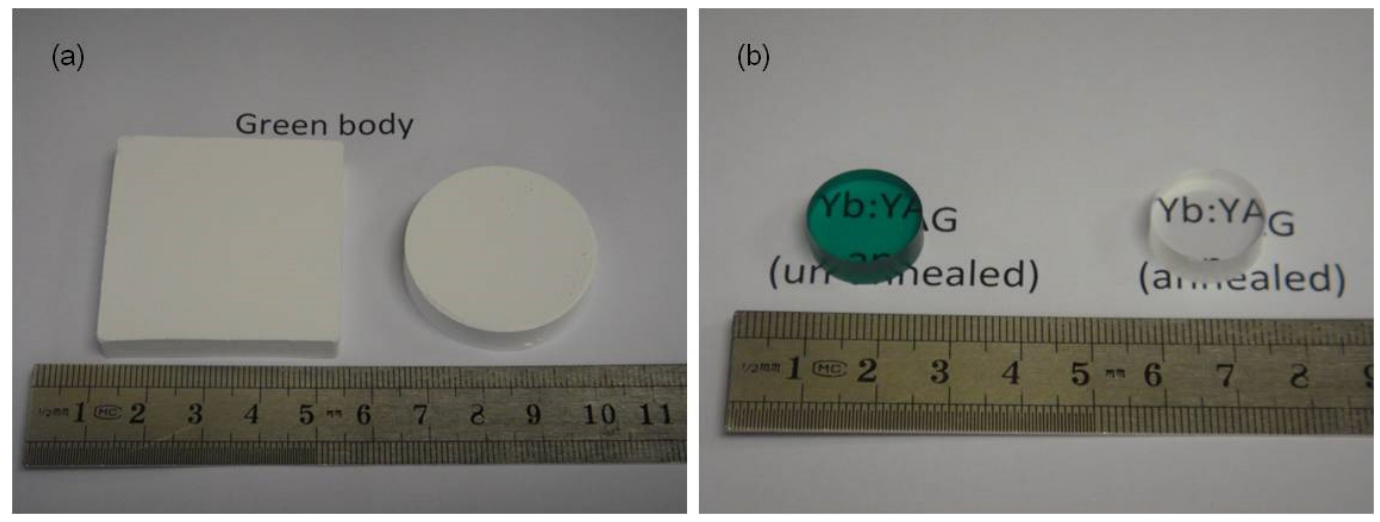

Figure 4.2 Photos of (a) green bodies and (b) sintered samples of the Yb:YAG ceramics.

Figure 4.2 shows the green bodies of sample before and after sintering. The green body density is measured to be about 55\% of the theoretical density value. Figure 4.2 (b) displays two polished 1.0 at.\% Yb:YAG ceramic samples, which are pellets of $16 \mathrm{~mm}$ in diameter and $3 \mathrm{~mm}$ in thickness. The thickness can be controlled through the grinding and polishing process. The as-sintered samples are green in color. They become colorless and transparent after annealing process.

\subsubsection{Characterization of Yb:YAG laser ceramics}

Microstructures morphologies of the raw powders and samples were inspected by using a scanning electron microscope (SEM, JSM-6360A, JEOL, Tokyo, Japan). The sintered samples for SEM observation were firstly polished and then thermally etched at $1500^{\circ} \mathrm{C}$ for $3 \mathrm{~h}$ to reveal the grain boundaries. Samples' densities were measured by Archimedes method. Crystalline phase compositions of the sintered samples were examined by using X-ray diffraction (XRD Bruker D8, Cu Ka radiation). Room temperature transmittance spectra of the polished samples were measured by using UV-VIS-NIR spectrophotometers (Carry 5000 Spectrophotometer, Varian, Australia). Optical Transmittance value $\mathrm{T}$ is defined by the ratio of light intensity after and before passing through the samples. It was measured and recorded by the spectrophotometer. Room temperature emission spectra were recorded by using a spectrofluorometer (Fluorolog-3, 
Jobin Yvon, Edison, USA) when samples were excited by a $940 \mathrm{~nm}$ wavelength laser diode. Solid-state laser systems were set up on optical tables for laser performance measurement.

\subsection{Results and Discussion}

\subsubsection{Phase and microstructure study}

SEM images of $\mathrm{Al}_{2} \mathrm{O}_{3}, \mathrm{Y}_{2} \mathrm{O}_{3}, \mathrm{Yb}_{2} \mathrm{O}_{3}$ raw powders and powder mixtures after ball milling are shown in Figure 4.3. The alumina oxide powder has a primary particle size of $\sim 150$ $\mathrm{nm}$. The $\mathrm{Y}_{2} \mathrm{O}_{3}$ raw powder shows heavy agglomeration. After ball milling, the powders appear homogenous, which proves the effectiveness of ball milling process.

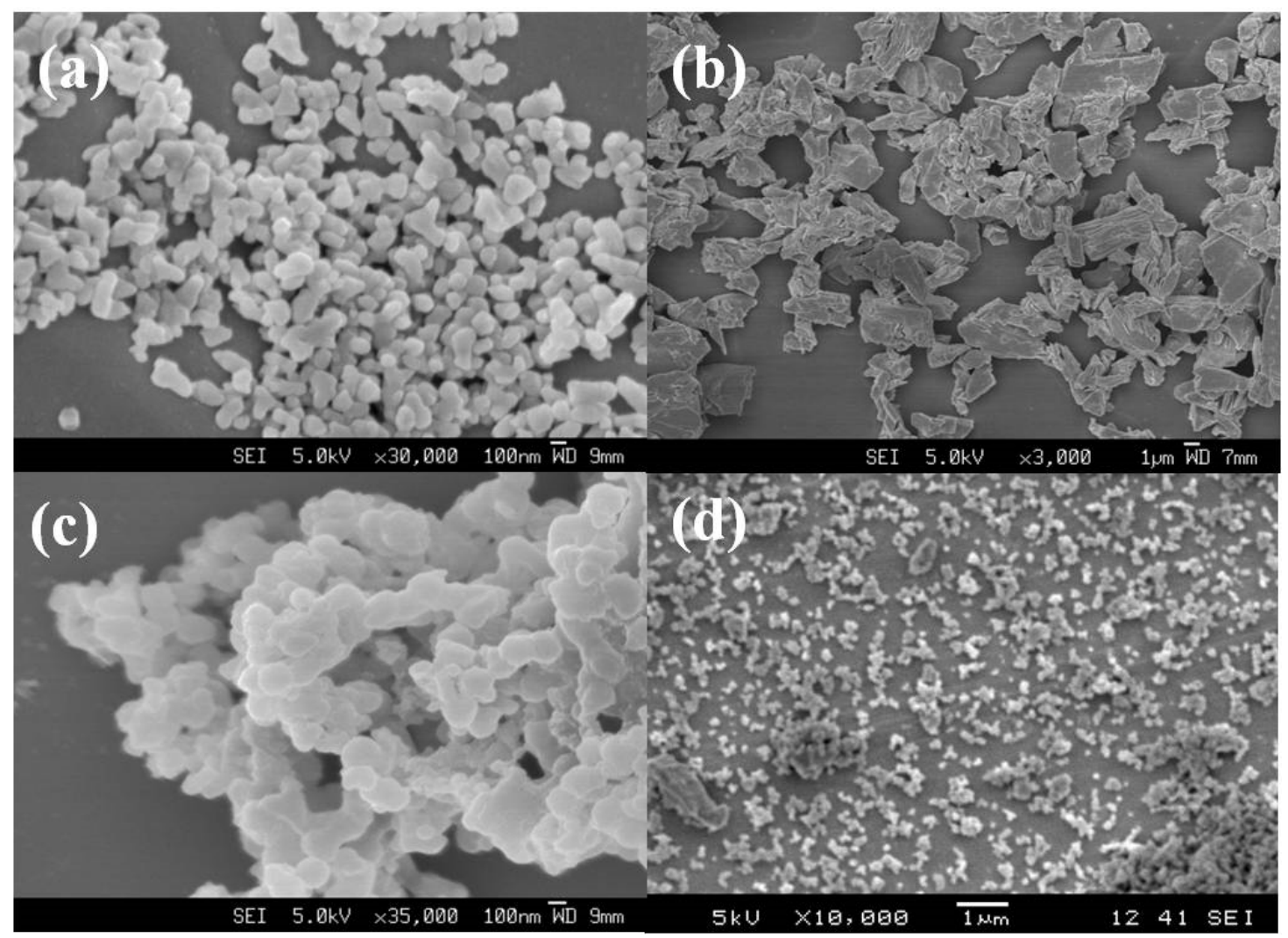

Figure 4.3 SEM images of raw powders: (a) $\mathrm{Al}_{2} \mathrm{O}_{3}$, (b) $\mathrm{Y}_{2} \mathrm{O}_{3}$, (c) $\mathrm{Yb}_{2} \mathrm{O}_{3}$ and (d) mixture after ball milling. 
Figure 4.4 shows the measured relative density and grain size for 5.0 at.\% Yb:YAG ceramics sintered at different temperature. The samples' relative density increases with the increment of sintering temperature. The relative density stays below $60 \%$ at $1400^{\circ} \mathrm{C}$ and increased rapidly at about $1600^{\circ} \mathrm{C}$ and reaches $98 \%$ by $1650^{\circ} \mathrm{C}$. With further incense of temperature, the density remains close to $100 \%$ of theoretical value.

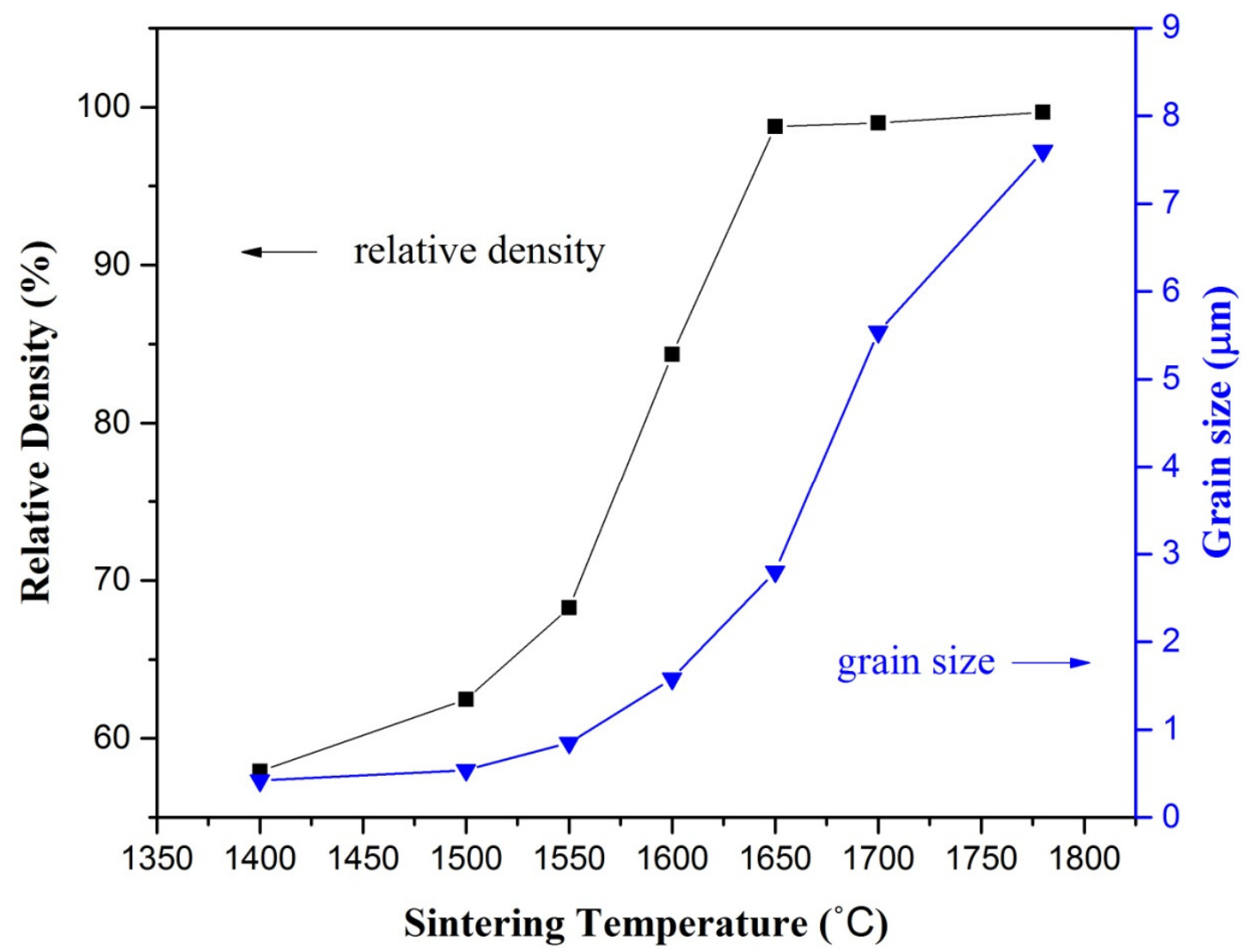

Figure 4.4 Relative density and grain size of 5.0 at.\% Yb:YAG ceramics.

The surface SEM images of samples sintered from $1400^{\circ} \mathrm{C}$ to $1700^{\circ} \mathrm{C}$ respectively are displayed in Figure 4.5. The samples had a very porous microstructure at the lower sintering temperature. Closed porosity appears at about $1550^{\circ} \mathrm{C}$ as shown in Figure 4.5 (c). Large pores are still observable at $1600^{\circ} \mathrm{C}$ in Figure 4.5 (d). With further increasing of sintering temperature to $1700^{\circ} \mathrm{C}$ in Figure 4.5 (f), the samples appeared fully dense and had almost pore-free microstructure. 

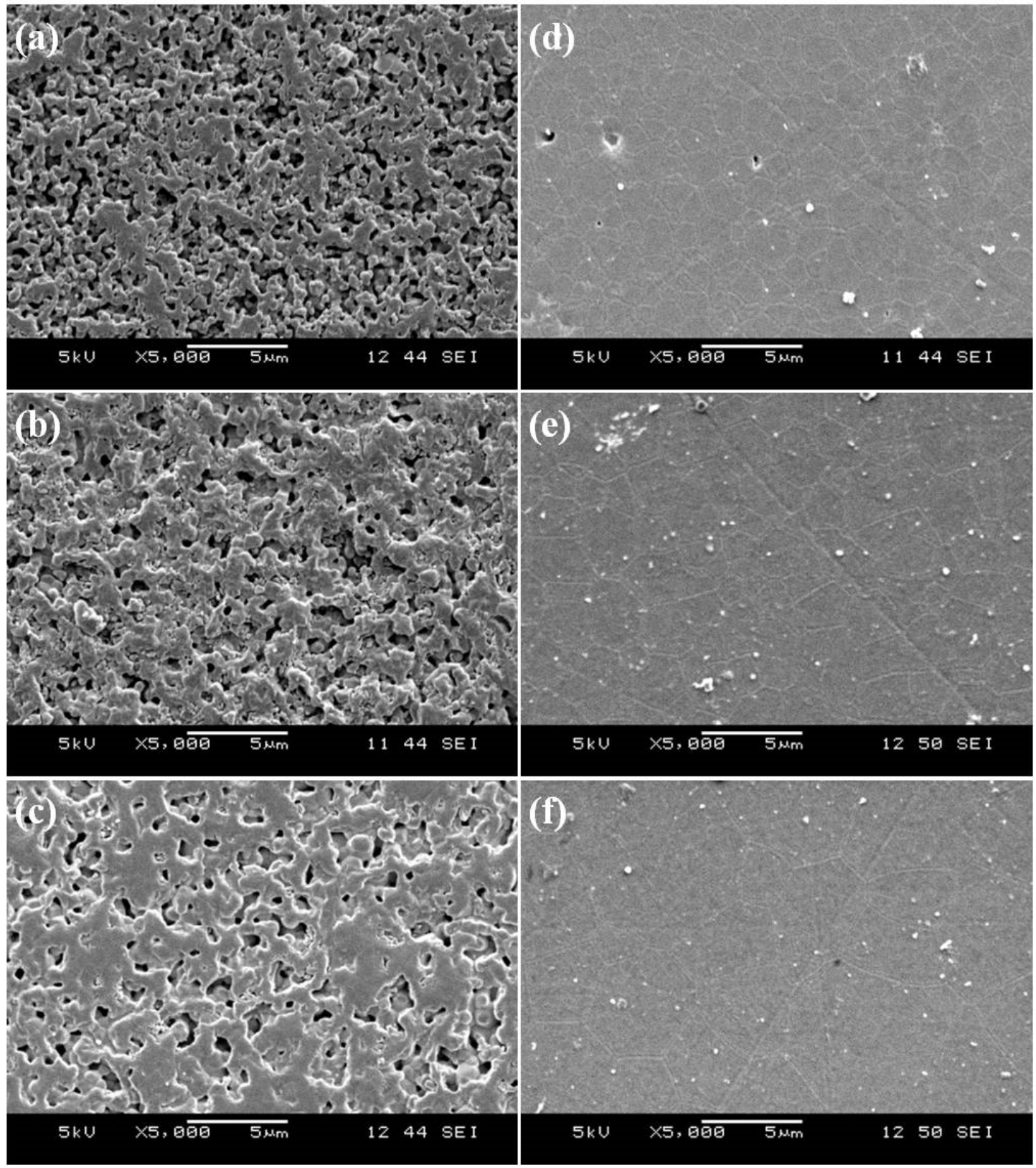

Figure 4.5 Surface SEM images of 5.0 at.\% Yb:YAG ceramics samples sintered at (a) $1400^{\circ} \mathrm{C}$ (b) $1500^{\circ} \mathrm{C}$, (c) $1550^{\circ} \mathrm{C}$ (d) $1600^{\circ} \mathrm{C}$ (e) $1650^{\circ} \mathrm{C}$ and (f) $1700^{\circ} \mathrm{C}$.

$\mathrm{XRD}$ patterns of the $\mathrm{Yb}$ :YAG ceramics of different doping $\mathrm{Yb}$ concentration is shown in Figure 4.6. The pattern shows that all the samples had a pure garnet phase. This indicates that $\mathrm{Yb}$ ion has been completely incorporated into the garnet lattice. There is no other phase detected by XRD. 


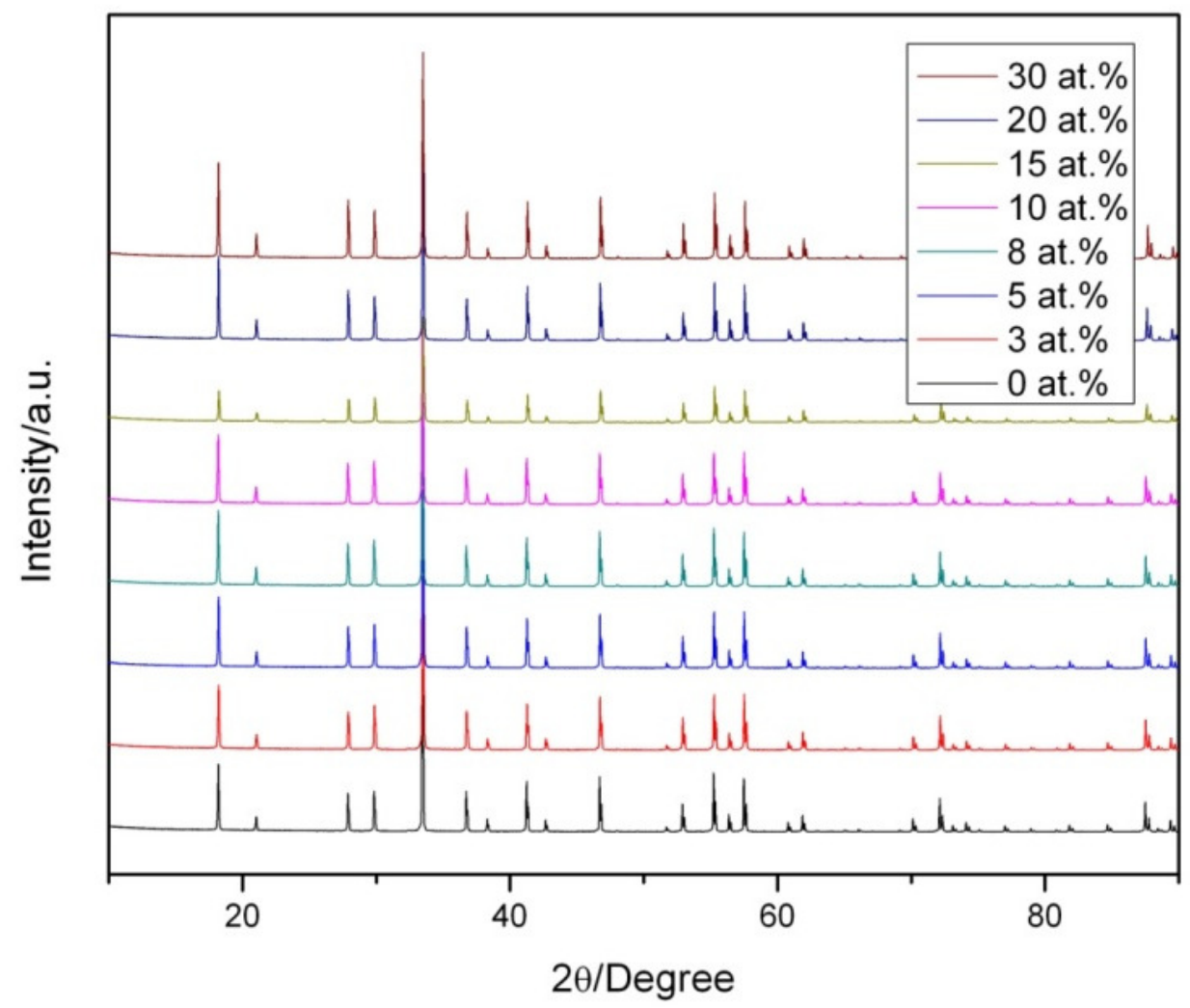

Figure 4.6 XRD patterns of $\mathrm{Yb}$ :YAG ceramics of different $\mathrm{Yb}$ doping concentration.

Thermal etched SEM images of the samples sintered at $1780^{\circ} \mathrm{C}$ with different $\mathrm{Yb}$ doping are displayed in Figure 4.7. Grain boundaries are clearly visible and all samples show an almost pore-free microstructure. The average grain size is about $10 \mu \mathrm{m}$. The smaller grain size means larger portion of grain boundaries, which could lead to more scattering loss if the grain boundaries were the main cause of light scattering. The grain size could be further controlled by adjusting the sintering time, sintering temperature and amount of sintering aids. Fracture SEM images are presented in Figure 4.8. It is observed that most fractures are transgranular with relatively smooth cross-section and less sharp grain edges. This indicates that the samples have relatively high grain boundary strength and toughness, which is a side reflection of good mechanical property of the samples. 

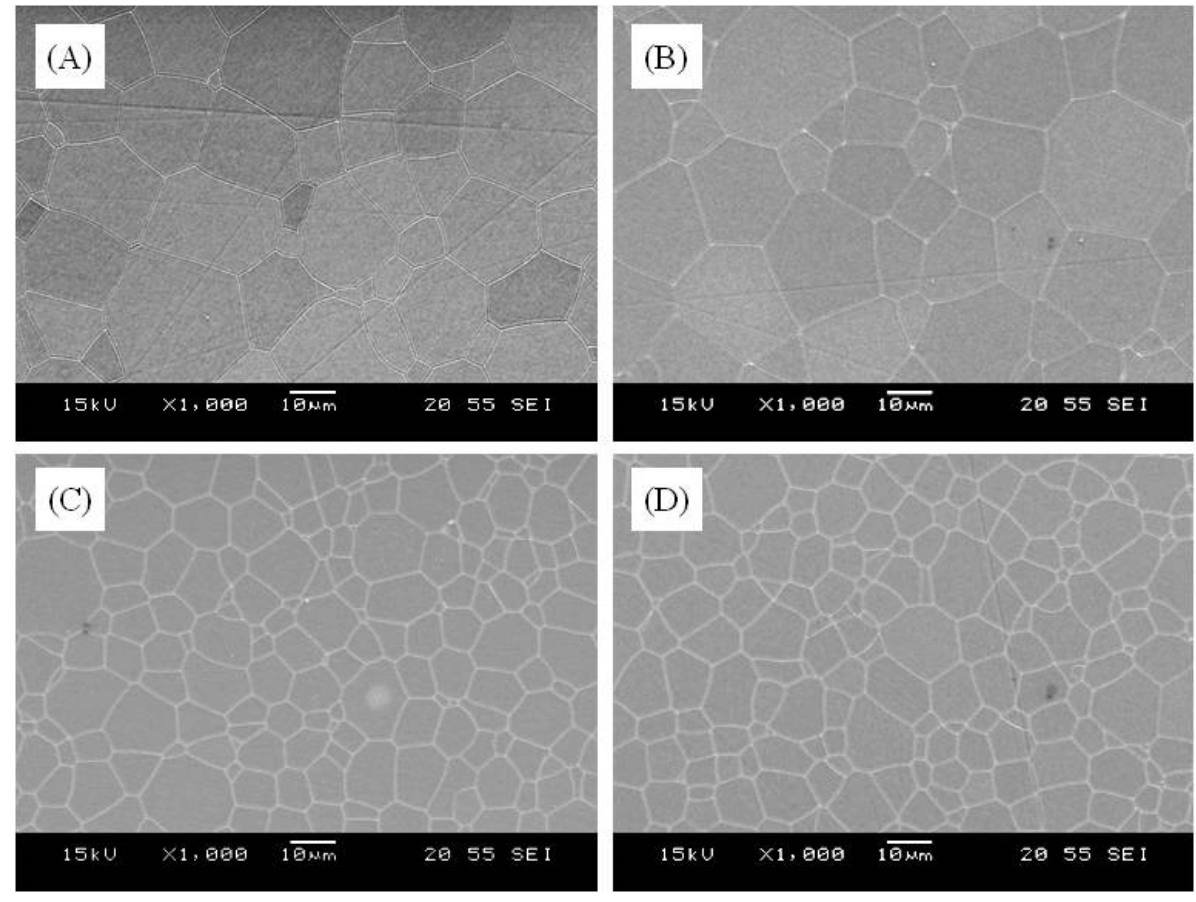

Figure 4.7 Thermal etched SEM images of the Yb:YAG ceramics with different doping concentrations: (A) 1 at.\%, (B) 8 at.\%, (C) 15 at.\% and (D) 20 at.\%.
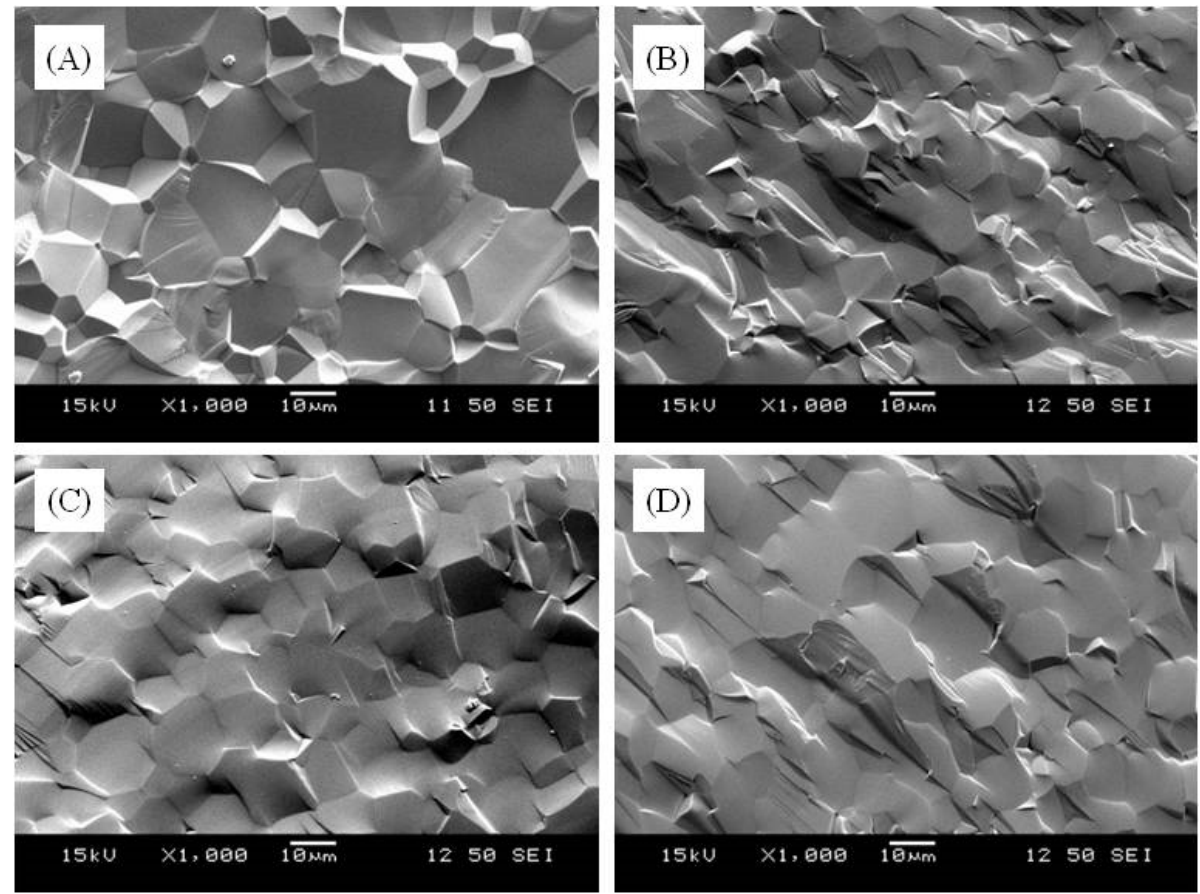

Figure 4.8 Fracture SEM images of the Yb:YAG ceramics with different doping concentrations: (A) 1 at.\%, (B) 8 at.\%, (C) 15 at.\% and (D) 20 at.\%. 


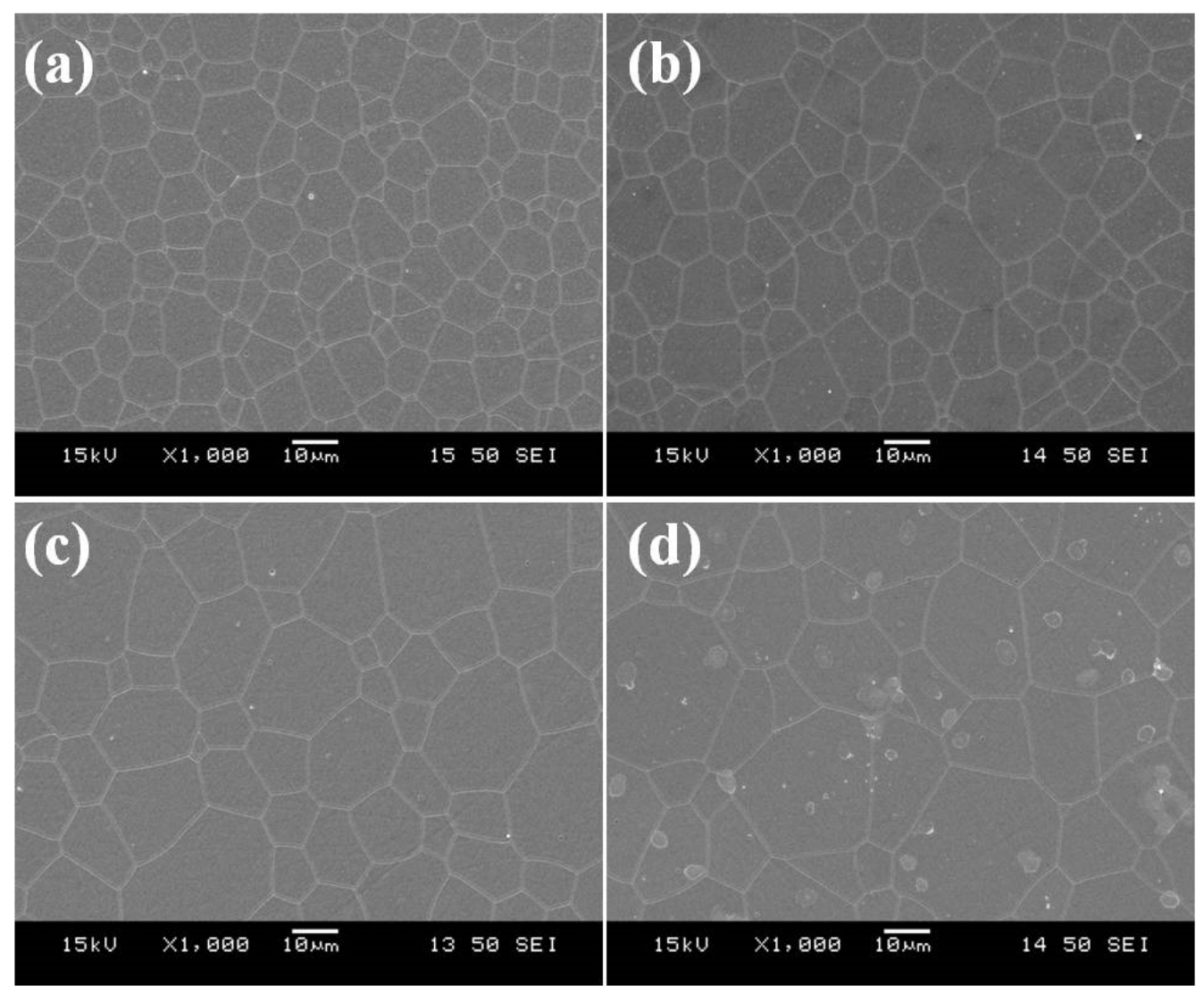

Figure 4.9 SEM images of the 5.0 at.\% Yb:YAG ceramics sintered for: (a) 8 h, (b) 12 $\mathrm{h},(\mathrm{c}) 24 \mathrm{~h}$ and (d) $30 \mathrm{~h}$.

Figure 4.9 shows the SEM images of the 5.0 at.\% Yb:YAG ceramics sintered at $1780^{\circ} \mathrm{C}$ for time durations of: $8 \mathrm{~h}, 12 \mathrm{~h}, 18 \mathrm{~h}, 24 \mathrm{~h}$ and $30 \mathrm{~h}$. Grain size grows with the prolonged sintering time. Larger grain size was observed with longer sintering time, The average grain size increased from $\sim 10 \mu \mathrm{m}$ of $8 \mathrm{~h}$ sintering time to $\sim 30 \mu \mathrm{m}$ of $30 \mathrm{~h}$ sintering time. No obvious pores are found within all of the samples. The optical study of the samples will be carried out in the next section.

\subsubsection{Optical and spectroscopic study}

After polishing of both surfaces, the room temperature in-line transmittance spectra of the samples were measured by spectrophotometer. Figure 4.10 presents the room temperature transmittance spectra of the 1.0 at.\% Yb:YAG ceramic sample before and after annealing 
for comparison. The wavelength range measured is from $200 \mathrm{~nm}$ to $1800 \mathrm{~nm}$. For the unannealed sample, two broad absorption bands at $375 \mathrm{~nm}$ and $625 \mathrm{~nm}$ are observed, which are ascribed to Re-F color centers. This is due to the presence of $\mathrm{Yb}^{2+}$ formed during the vacuum sintering [34, 35]. The two absorption bands completely disappeared after annealing. For the annealed sample, the transmittance reaches $83.6 \%$ at $1300 \mathrm{~nm}$ wavelength, which is very close to its theoretical value. The transmittance remains $81.8 \%$ at $400 \mathrm{~nm}$ wavelength, indicating its good optical quality.

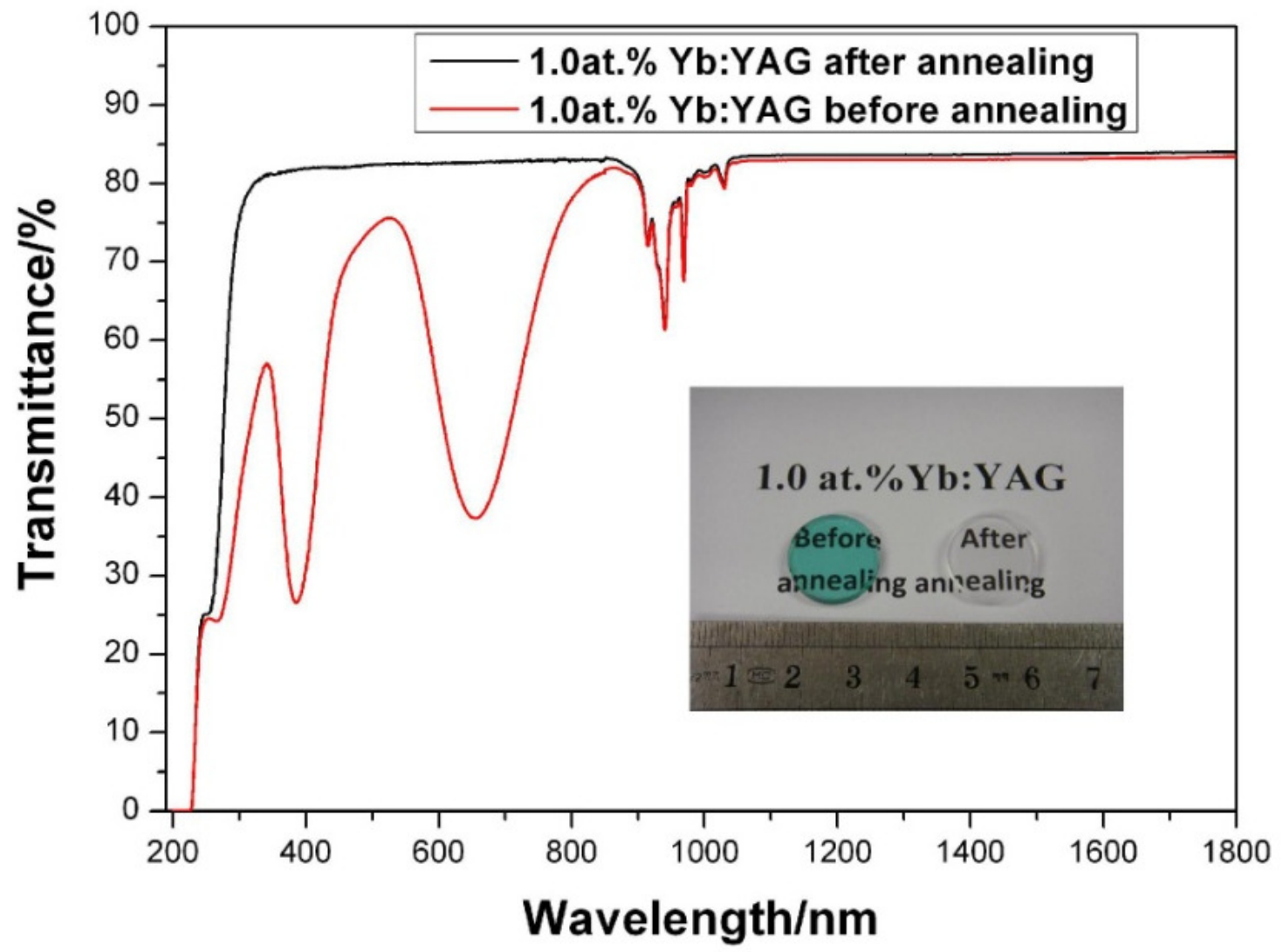

Figure 4.10 Transmittance curves of the 1.0 at.\% Yb:YAG ceramics before and after annealing, with their photographs shown in the inset. 


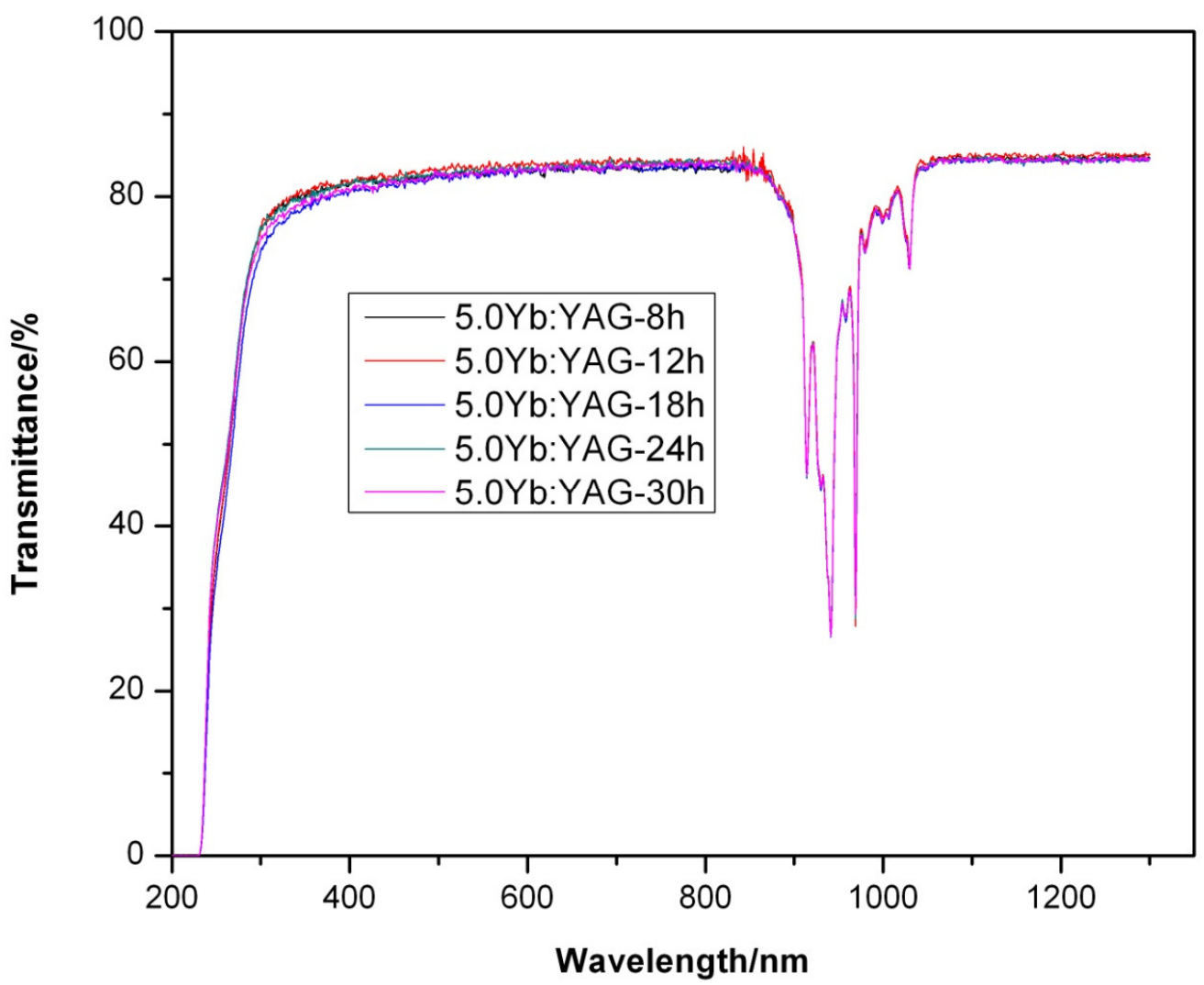

Figure 4.11 Transmittance curves of the 5.0 at.\% Yb:YAG ceramics with different sintering time durations.

Figure 4.11 shows the transmittance curves of the 5.0 at.\% Yb:YAG ceramics sintered at $1780^{\circ} \mathrm{C}$ with sintering time ranging from $8 \mathrm{~h}$ to $30 \mathrm{~h}$. All the samples have transmittance value above $81 \%$ at $400 \mathrm{~nm}$ and $84 \%$ at $1300 \mathrm{~nm}$ wavelength. For absorption band, the absorption curves coincide with each other. No obvious difference was observed. This result shows that sintering time of longer than 8 h has no obvious effect on the samples' optical property under the same sintering temperature and condition. So prolonged sintering is unnecessary for improvement in sample's optical quality in terms of costeffectiveness.

Optical transmittances of the annealed samples with different $\mathrm{Yb}$ doping concentrations are plotted in Figure 4.12. All samples are polished to $3 \mathrm{~mm}$ in thickness. It is observed that the main absorption band of the Yb:YAG ceramics is from $850 \mathrm{~nm}$ to $1050 \mathrm{~nm}$. The 
transmittance at $940 \mathrm{~nm}$ drops to a very low value for high doping samples due to the strong absorption. At non-absorption region, the transmittance values all remain above $81 \%$ regardless of the doping concentration.

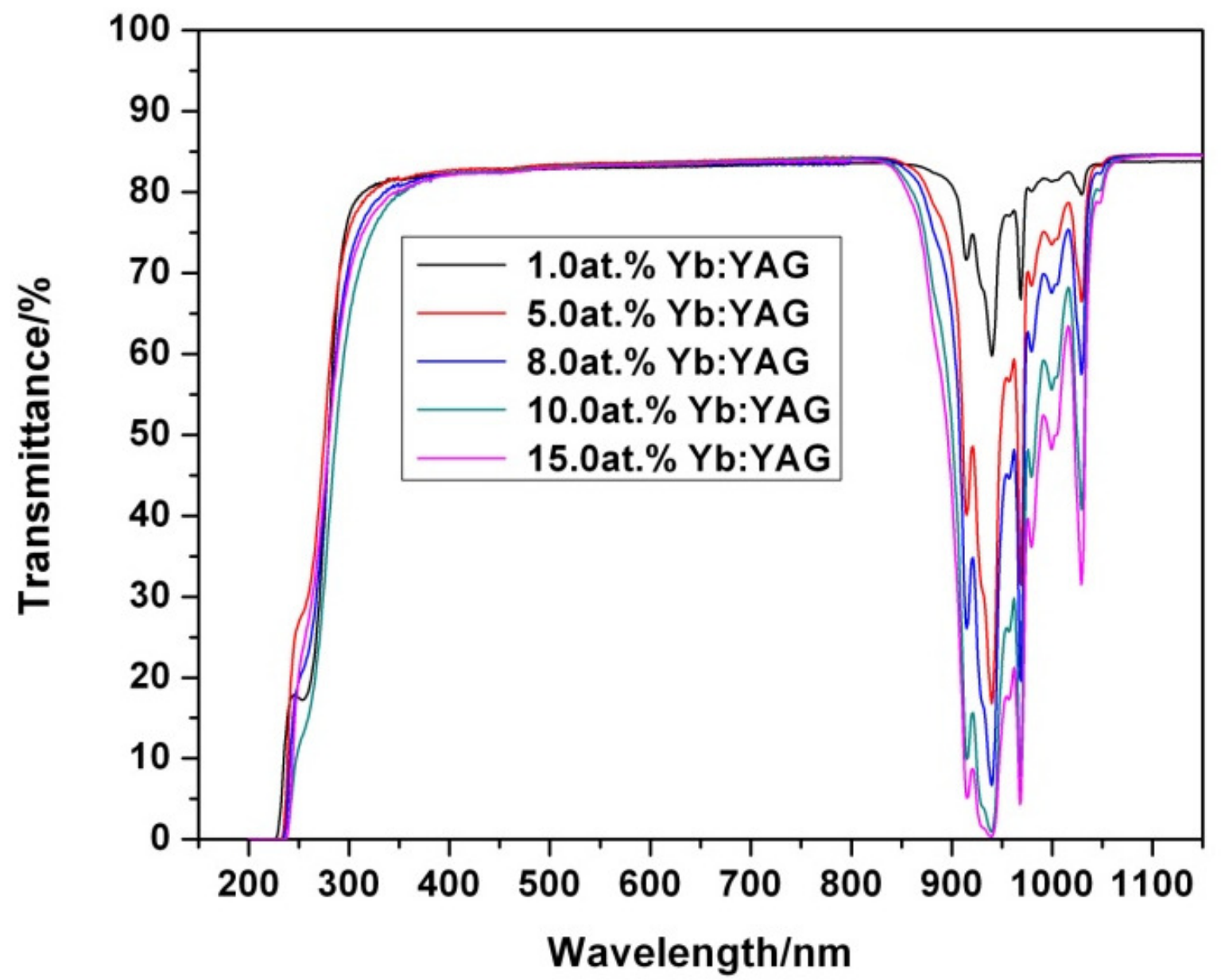

Figure 4.12 Transmittance curves of the Yb:YAG ceramic samples (annealed) with various doping concentrations.

With the transmittance date of the Yb:YAG samples available, the absorption coefficient, $\alpha$, of the respective samples can be calculated by using the following equation:

$$
\alpha=\frac{1}{L} \ln \left(\frac{(1-R)^{2}}{2 T}+\sqrt{R^{2}+\frac{(1-R)^{4}}{4 T^{2}}}\right)
$$

where $\mathrm{L}$ is sample thickness, $\mathrm{T}$ is transmittance value, and $\mathrm{R}$ is surface reflectance, which 
is defined as:

$R=\left(\frac{n-1}{n+1}\right)^{2}$

where $\mathrm{n}$ is refractive index, which is 1.819 for YAG material at $1 \mu \mathrm{m}$ wavelength. Calculated absorption spectra of the samples from $800 \mathrm{~nm}$ to $1100 \mathrm{~nm}$ wavelength are plotted in Figure 4.13.

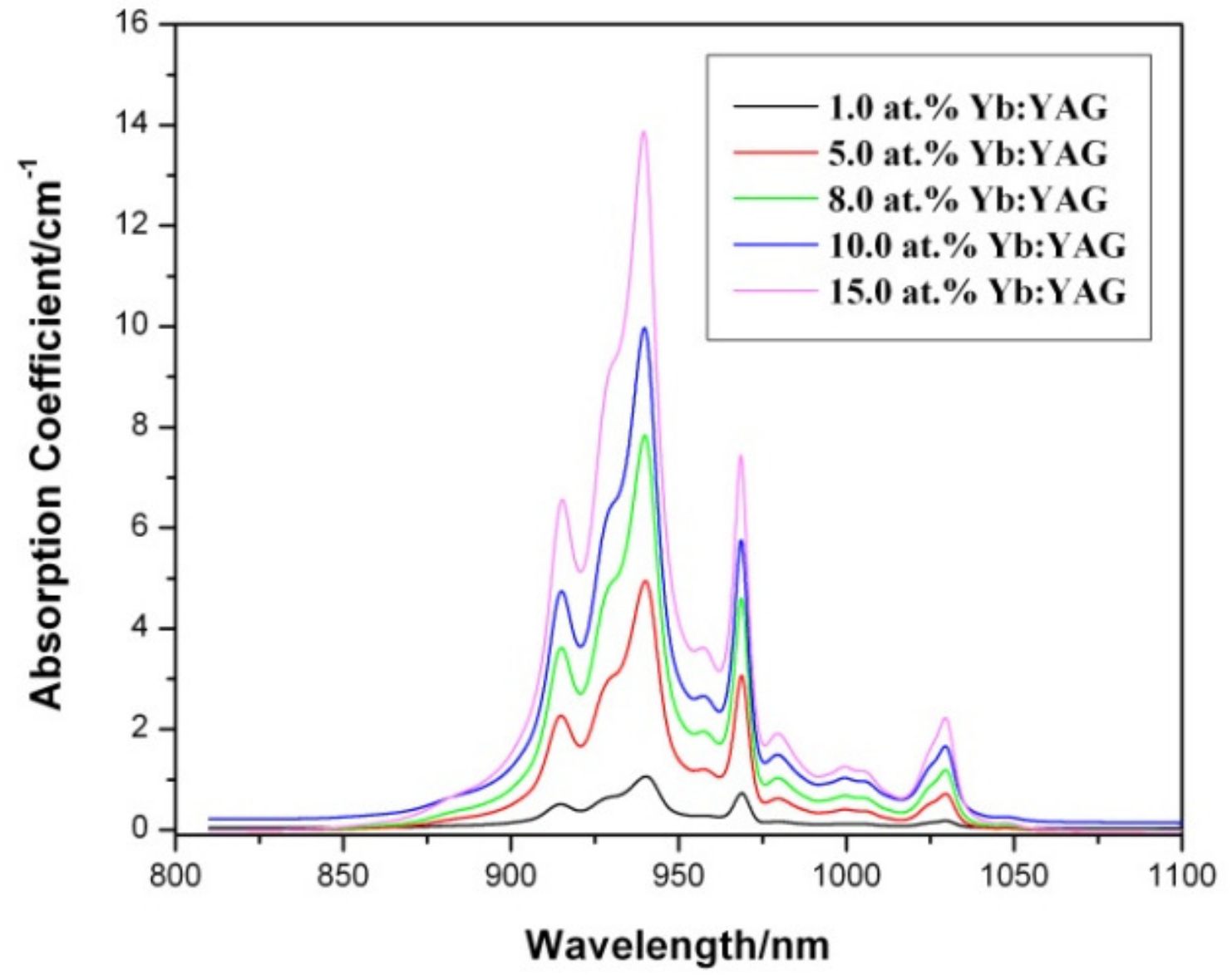

Figure 4.13 Absorption spectra of the Yb:YAG ceramics with different $\mathrm{Yb}$ doping concentrations.

From the absorption spectra of the Yb:YAG ceramics with different dopings, it is observed that all samples have a very strong absorption peak at $940 \mathrm{~nm}$ and two moderate absorption peaks at $915 \mathrm{~nm}$ and $969 \mathrm{~nm}$, respectively, corresponding to the ${ }^{2} \mathrm{~F}_{7 / 2} \rightarrow{ }^{2} \mathrm{~F}_{5 / 2}$ transition of $\mathrm{Yb}^{3+}$ ion. The absorption coefficient at $940 \mathrm{~nm}$ increases from $1.06 \mathrm{~cm}^{-1}$ for 
the 1.0 at.\% Yb:YAG sample to $13.87 \mathrm{~cm}^{-1}$ for the 15.0 at.\% one. Re-absorption at 1030 $\mathrm{nm}$ lasing wavelength is also clearly observed, which is attributed to the quasi-three-level nature of the ytterbium-doped gain media.

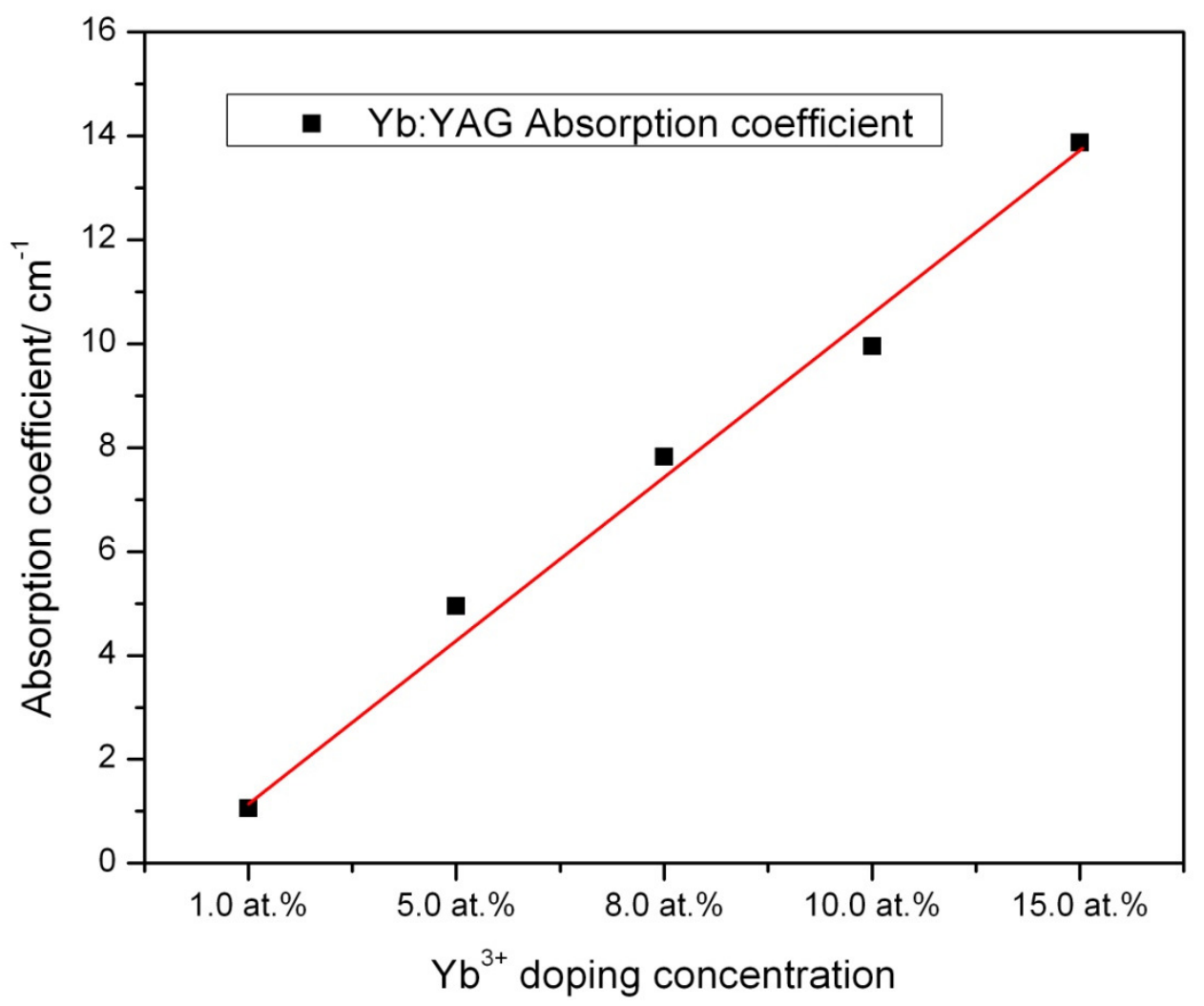

Figure 4.14 Absorption coefficient of Yb:YAG ceramics at $940 \mathrm{~nm}$ with respective to the doping concentration.

Figure 4.14 shows the absorption coefficients of the Yb:YAG ceramics samples at $940 \mathrm{~nm}$ wavelength with respective to the doping concentration. The absorption coefficient increases almost linearly with increment of $\mathrm{Yb}^{3+}$ concentration. This is because higher doping concentration implies high $\mathrm{Yb}^{3+}$ ion density in the material, leading to a stronger absorption effect. 

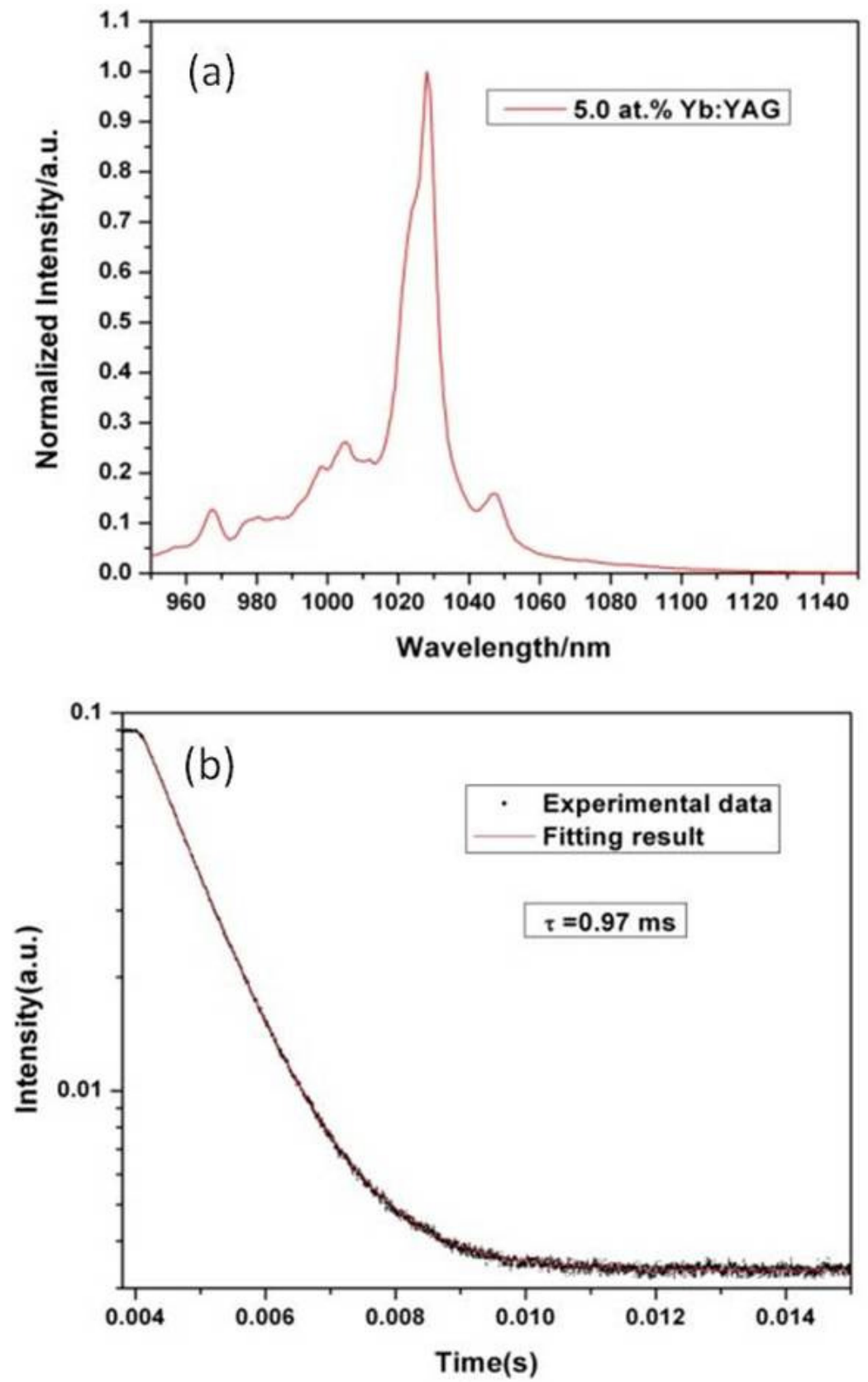

Figure 4.15 The (a) fluorescence spectrum and (b) fluorescence decay curve of the 5.0 at. $\% \mathrm{Yb}: \mathrm{YAG}$ ceramics.

The fluorescent spectrum and fluorescence decay curve of the 5.0 at.\% Yb:YAG ceramic sample is plotted in Figure 4.15 (a) and (b) respectively. The sample was excited by a 940 $\mathrm{nm}$ laser diode during measurement for efficient absorption. A strong emission peak is located at $1028 \mathrm{~nm}$ wavelength. Full width at half maximum (FWHM) at the emission 
peak is measured to be $11.07 \mathrm{~nm}$. The wide emission bandwidth is favorable for generating ultra short pulse in mode-locked laser operations. From the fluorescence decay curve, the fluorescent lifetime was measured to be $0.97 \mathrm{~ms}$. The long fluorescent lifetime is favorable for higher energy storage in high power laser operations. However, radiation trapping and re-absorption effect will cause significant lengthening of the measurement result [36], which will lead to an underestimated calculated emission cross-section. Further improvement, such as using very thin piece of samples, could reduce the reabsorption effect to some extent and improve the measurement accuracy.

Absorption and emission cross-section are intrinsic characteristics of materials, which are independent of doping concentration. Taking the 5.0 at.\% Yb:YAG sample for consideration, the absorption cross-section can be calculated by using the following equation:

$\sigma_{a b s}(\lambda)=\frac{\alpha M}{\rho N_{A} C}$

where $\alpha$ is absorption coefficient, $\mathrm{M}$ is molar mass of the 5.0 at.\% Yb:YAG, which is $606.23 \mathrm{~g} / \mathrm{mol}, \rho$ is density of the sample, which is $4.55 \mathrm{~g} / \mathrm{cm}^{3}, \mathrm{~N}_{\mathrm{A}}$ stands for Avogadro's Number and $\mathrm{C}$ is $\mathrm{Yb}$ ion concentration.

The emission cross-section can be calculated by using the Fuchtbauer-Landenburg formula [37]:

$$
\sigma_{e m}(\lambda)=\frac{\lambda^{5} I(\lambda)}{8 \pi n^{2} c \tau \int I(\lambda) \lambda d \lambda}
$$

where $I(\lambda)$ is intensity of emission spectrum, $\tau$ is the measured fluorescence lifetime, $n$ is refractive index of the sample which is 1.819 and $\mathrm{c}$ is light velocity. 


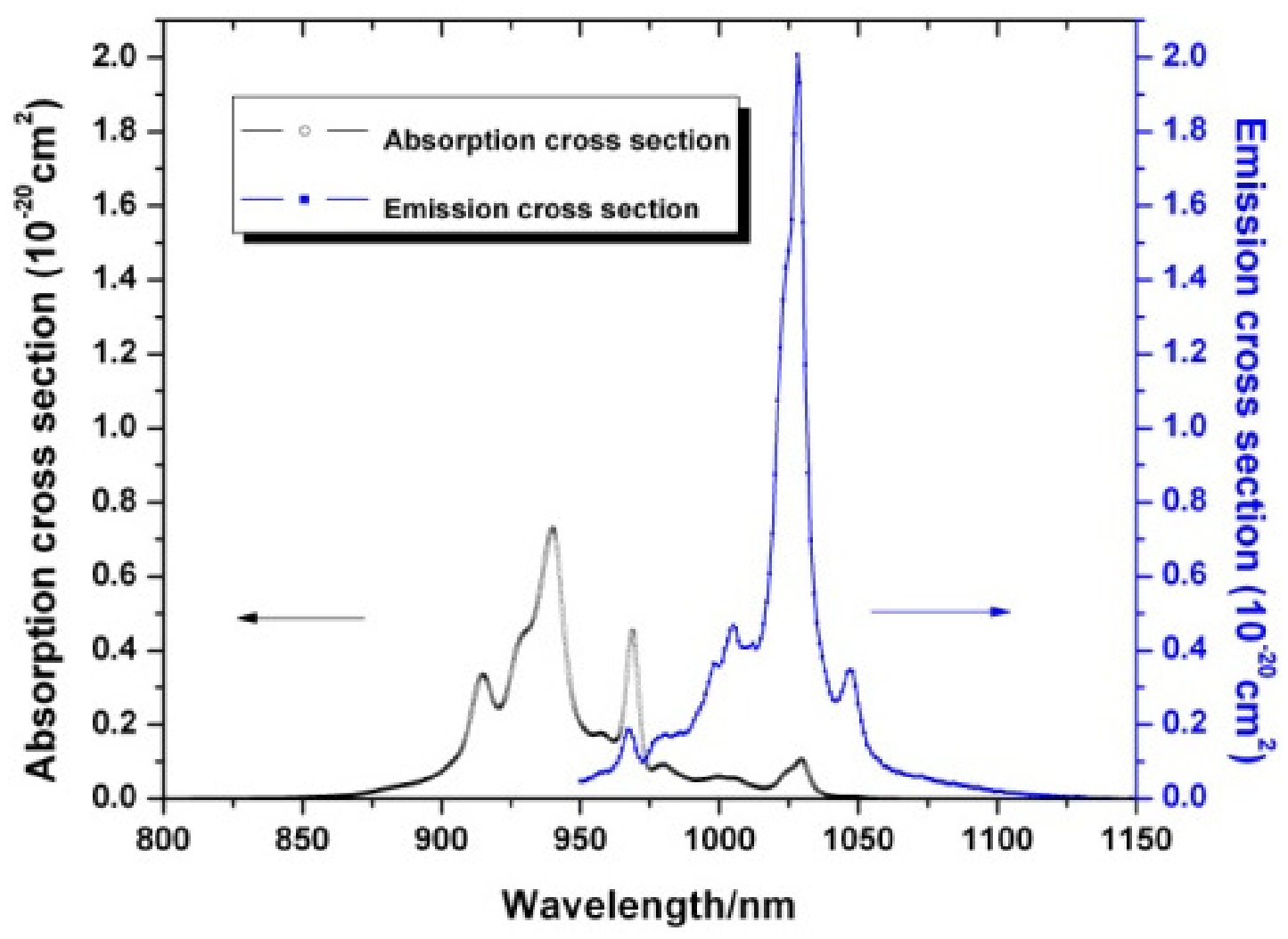

Figure 4.16 Absorption and emission cross-section of the Yb:YAG ceramics.

Figure 4.16 displays the calculated absorption and emission cross-section of the Yb:YAG ceramics. $\sigma_{\text {abs }}$ value at $940 \mathrm{~nm}$ absorption peak is $0.72 \times 10^{-20} \mathrm{~cm}^{2}$ and $\sigma_{\mathrm{em}}$ at $1028 \mathrm{~nm}$ emission peak is $2.01 \times 10^{-20} \mathrm{~cm}^{2}$. The $\sigma_{\mathrm{em}}$ value is comparable with that of $\mathrm{Yb}$ :YAG single crystal reported [37].

Gain cross-section of the Yb:YAG ceramics was then determined under different population inversion ratio, $\beta$, which equals to $\mathrm{N} 2 / \mathrm{N} 0$, where $\mathrm{N} 2$ is inversion population number and N0 is total population number. The gain cross-section, $\sigma_{\mathrm{g}}(\lambda)$, was calculated by:

$\sigma_{g}(\lambda)=\beta \times \sigma_{e m}(\lambda)-(1-\beta) \times \sigma_{a b s}(\lambda)$

where $\sigma_{\mathrm{em}}(\lambda)$ and $\sigma_{\mathrm{abs}}(\lambda)$ are the emission and absorption cross-section, respectively. The gain cross-section of the Yb:YAG ceramics under different $\beta$ was plotted in Figure 4.17. 


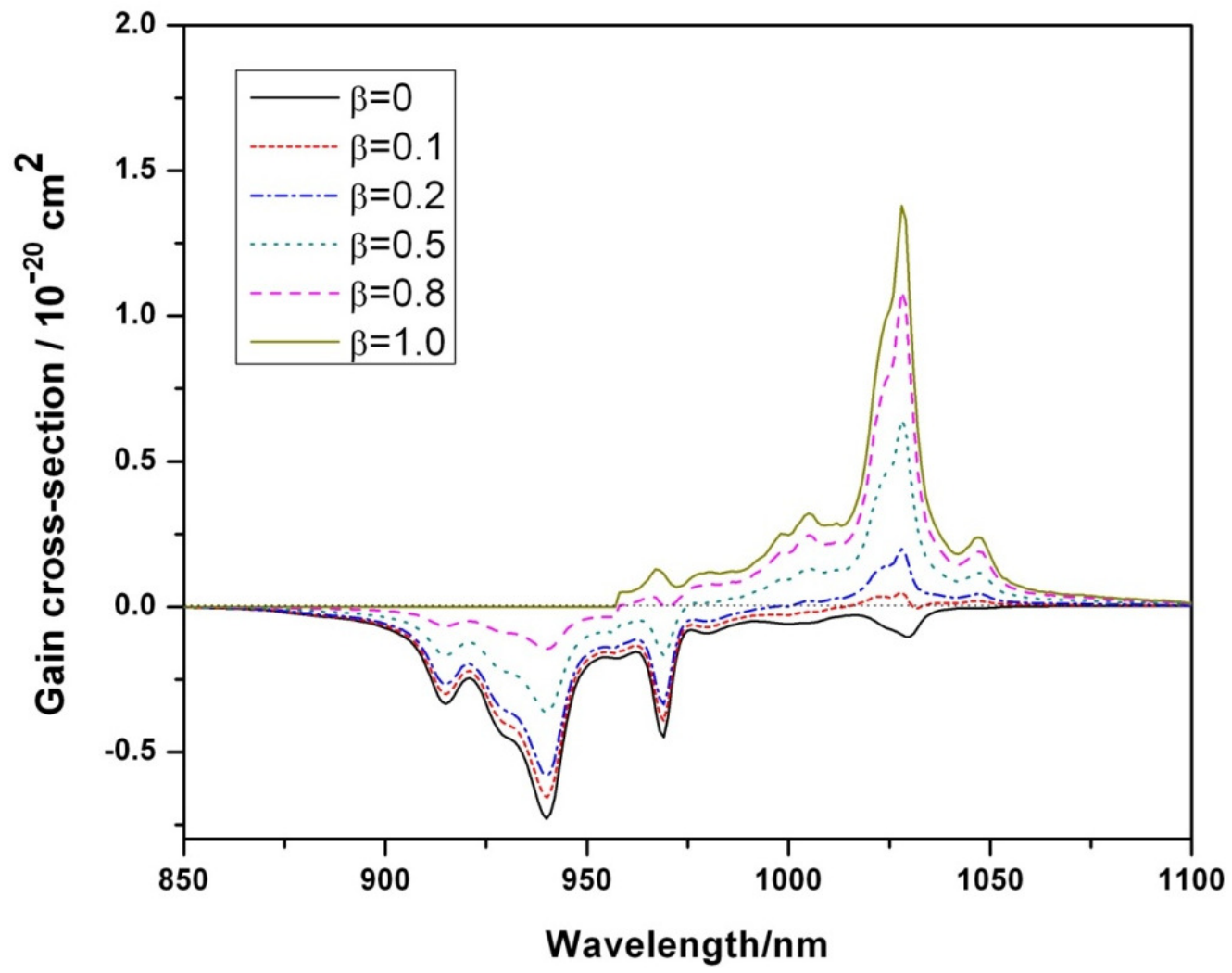

Figure 4.17 Gain cross-section of the Yb:YAG ceramics under different $\beta$.

\subsubsection{Solid state laser performance}

In order to test laser performances of the samples, an end-pumped two mirror cavity was used. The cavity configuration is shown in Figure 4.18. The input mirror was a plano mirror and the output coupler had a radius of curvature (ROC) of $50 \mathrm{~mm}$, which forms a cavity of $15 \mathrm{~mm}$ in length. The pump source was a $25 \mathrm{~W} 940 \mathrm{~nm}$ fiber coupled laser diode (Limo-25-f100-d1940) with a fiber core diameter of $100 \mu \mathrm{m}$. The pump beam was focused on the Yb:YAG ceramic sample with lens of a focal length of $30 \mathrm{~mm}$. The sample was cut into a $3 \times 3 \times 3.2 \mathrm{~mm}^{3}$ cube and anti-reflection coated on both sides $(3 \times 3$ $\mathrm{mm}^{2}$ ) at the pump and lasing wavelength to reduce reflection loss. The sample was wrapped with indium foil, placed in a copper holder which was water cooled at $18^{\circ} \mathrm{C}$. The cooling water was used to dissipate the heat generated during laser operation to prevent thermal damage of the sample. 


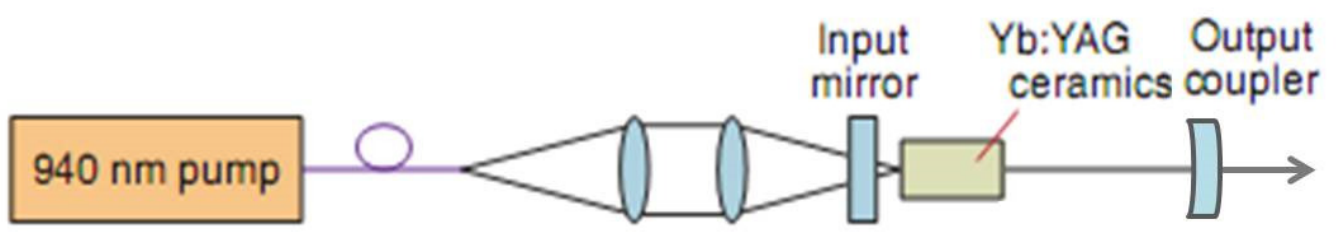

Figure 4.18 The cavity configuration of a CW Yb:YAG ceramics laser.

During laser operation, the output power was recorded by a power-meter at far right side of lasing direction, while the pump source increased the input power. Residual power was measured without the output coupler (i.e. without lasing condition). Absorbed pump power was calculated by the input power minus the residual power.

CW laser operation was successfully achieved for all the samples with different doping concentrations by using two different output couplers. The laser performance of output power versus absorbed power is shown in Figure 4.19. A maximum laser output power of $6.9 \mathrm{~W}$ was obtained by using the 8.0 at.\% Yb:YAG ceramics sample. Highest slope efficiency of $62.7 \%$ was obtained by using the 5.0 at.\% Yb:YAG ceramic sample. The laser threshold was $1.3 \mathrm{~W}$. It is observed that the samples with higher doping levels showed relatively lower laser output power. This is mainly because that all samples used in the experiment had the same thickness. For samples with higher doping, most of the input laser energy is absorbed at their front part. The rest part of the samples became redundant and was not good for heat dissipation. This resulted in lower output power and efficiency. No saturation was observed in the experiment, which means that there is still possibility to further increase the laser output power. However it is not realized due to the equipment limitation. It is also believed that, with further reduction in thickness of the samples with different doping concentrations, higher output power and slope efficiency can be achieved. 

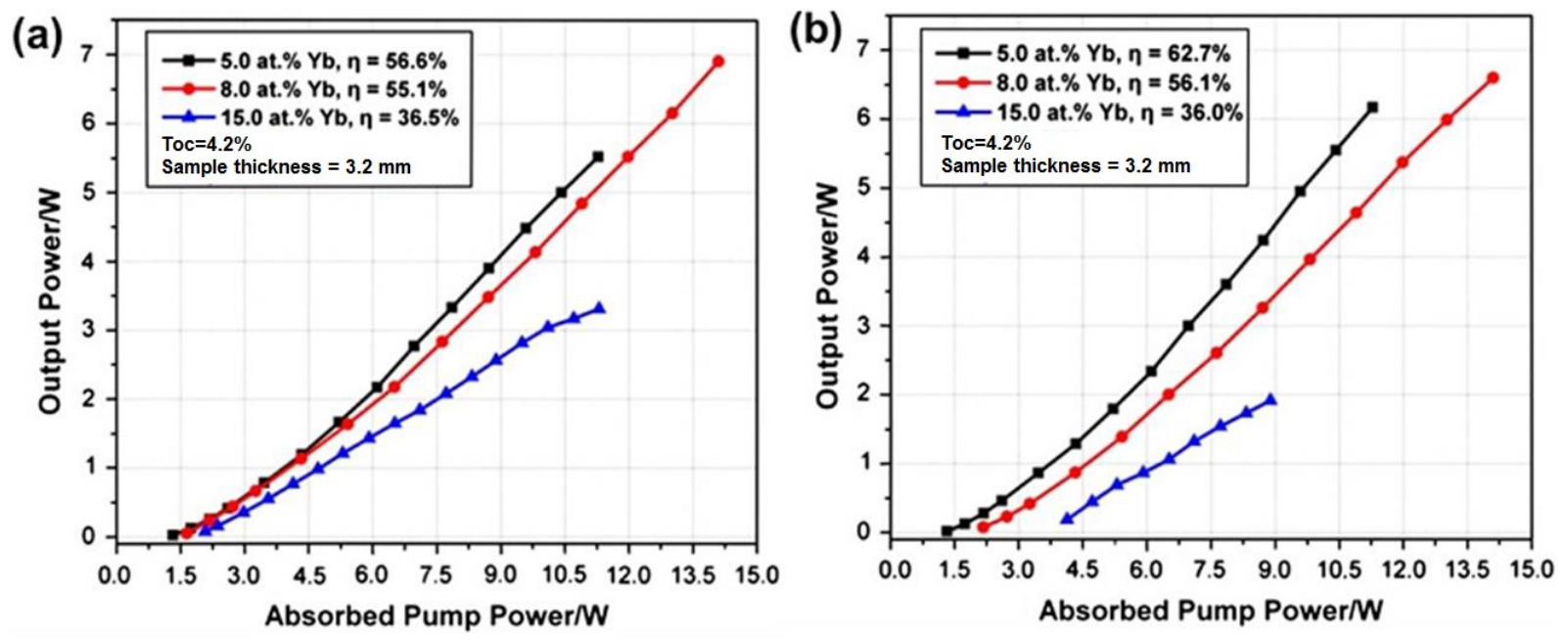

Figure 4.19 Laser performance of the Yb:YAG ceramics.

\subsection{Conclusions}

In this chapter, efforts have been focused on development of Yb:YAG garnet transparent laser ceramics. The fabrication method has been established by using commercial powder as raw material and sintered under reactive sintering method. Microstructure and densification process of samples were also studied. Spectroscopic analysis and laser properties of fabricated samples were tested. High quality Yb:YAG ceramics have been successfully obtained, with which laser performance has been realized. Compared with their single crystal counterparts, polycrystalline ceramics laser materials could be fabricated in a relatively shorter period at lower production cost. It is also more convenient to realize high doping, large-size, multilayer and multifunctional materials by using laser ceramics for practical laser applications. Because of these remarkable advantages they possess, the Yb:YAG laser ceramics will show great potential to replace their single crystal counterparts in the $1 \mu \mathrm{m}$ laser application, especially in thin disk laser system. For example, Perevezentsev et al. had obtained a $233 \mathrm{~mJ}$ output laser power at $143 \mathrm{~Hz}$ for a cryogenic Yb:YAG disk laser using the 5 at.\% doped Yb:YAG ceramics samples provided by the author [38]. The output result is the best among all high repetition rate cryogenic pulse Yb:YAG ceramic thin disk laser systems. Further 
improvement in the optical-to-optical efficiency is still possible by optimization in the ceramics' optical quality as well as the doping design.

\section{References}

[1] G. Bogomolova, D. Vylegzhanin, and A. Kaminskii, Soviet Journal of Experimental and Theoretical Physics. 1976, 42, 440.

[2] W. F. Krupke, IEEE Journal of Selected Topics in Quantum Electronics. 2000, 6, 1287-1296.

[3] T. Taira, W. M. Tulloch, and R. L. Byer, Applied Optics. 1997, 36, 1867-1874.

[4] T. Fan, IEEE Journal of Quantum Electronics. 1992, 28, 2692-2697.

[5] H. W. Bruesselbach, D. S. Sumida, R. A. Reeder, and R. W. Byren, IEEE Journal of Selected Topics in Quantum Electronics. 1997, 3, 105-116.

[6] P. Yang, P. Deng, and Z. Yin, Journal of Luminescence. 2002, 97, 51-54.

[7] J. Dong, A. Shirakawa, K. Ueda, H. Yagi, T. Yanagitani, and A. A. Kaminskii, Optics Letters. 2007, 32, 1890-1892.

[8] F. D. Patel, E. C. Honea, J. Speth, S. A. Payne, R. Hutcheson, and R. Equall, IEEE Journal of Quantum Electronics. 2001, 37, 135-144.

[9] H. Bruesselbach, and D. S. Sumida, IEEE Journal of Selected Topics in Quantum Electronics. 2005, 11, 600-603.

[10] E. Innerhofer, T. Südmeyer, F. Brunner, R. Häring, A. Aschwanden, R. Paschotta, C. Hönninger, M. Kumkar, and U. Keller, Optics Letters. 2003, 28, 367-369.

[11] P. Lacovara, H. Choi, C. Wang, R. Aggarwal, and T. Fan, Optics Letters. 1991, 16, 1089-1091.

[12] J. Dong, M. Bass, Y. Mao, P. Deng, and F. Gan, Journal of the Optical Society of America B. 2003, 20, 1975-1979.

[13] U. Brauch, A. Giesen, M. Karszewski, C. Stewen, and A. Voss, Optics Letters. 1995, 20, 713-715.

[14] T. Y. Fan, S. Klunk, and G. Henein, Optics Letters. 1993, 18, 423-425.

[15] J. A. der Au, G. Spühler, T. Südmeyer, R. Paschotta, R. Hövel, M. Moser, S. Erhard, M. Karszewski, A. Giesen, and U. Keller, Optics Letters. 2000, 25, 859-861. 
[16] D. J. Ripin, J. R. Ochoa, R. Aggarwal, and T. Y. Fan, Optics Letters. 2004, 29, 2154-2156.

[17] J. A. der Au, S. Schaer, R. Paschotta, C. Hönninger, U. Keller, and M. Moser, Optics Letters 1999, 24, 1281-1283.

[18] E. C. Honea, R. J. Beach, S. C. Mitchell, J. A. Skidmore, M. A. Emanuel, S. B. Sutton, S. A. Payne, P. V. Avizonis, R. S. Monroe, and D. G. Harris, Optics Letters. 2000, 25, 805-807.

[19] T. Taira, J. Saikawa, T. Kobayashi, and R. L. Byer, IEEE Journal of Selected Topics in Quantum Electronics. 1997, 3, 100-104.

[20] J. G. Manni, J. D. Hybl, D. Rand, D. J. Ripin, J. R. Ochoa, and T. Y. Fan, IEEE Journal of Quantum Electronics. 2010, 46, 95-98.

[21] Y. Wu, J. Li, Y. Pan, J. Guo, B. Jiang, Y. Xu, and J. Xu, Journal of the American Ceramic Society. 2007, 90, 3334-3337.

[22] Y. Wu, J. Li, F. Qiu, Y. Pan, Q. Liu, and J. Guo, Ceramics International. 2006, 32, 785-788

[23] J. Dong, K. Ueda, H. Yagi, A. Kaminskii, and Z. Cai, Laser Physics Letters. 2008, 6, 282.

[24] K. Takaichi, H. Yagi, J. Lu, A. Shirakawa, K. Ueda, T. Yanagitani, and A. Kaminskii, Physica Status Solidi (a). 2003, 200, R5-R7.

[25] J. Dong, A. Shirakawa, K. Ueda, H. Yagi, T. Yanagitani, and A. A. Kaminskii, Applied Physics Letters. 2006, 89, 91114-91114.

[26] S. Nakamura, H. Yoshioka, Y. Matsubara, T. Ogawa, and S. Wada, Optics Communications. 2008, 281, 4411-4414.

[27] J. Zhou, W. Zhang, T. Huang, L. Wang, J. Li, W. Liu, B. Jiang, Y. Pan, and J. Guo, Ceramics International. 2011, 37, 513-519.

[28] F. Tang, Y. Cao, J. Huang, H. Liu, W. Guo, and W. Wang, Journal of the American Ceramic Society 2012, 95, 56-69.

[29] J. Kawanaka, N. Miyanaga, T. Kawashima, K. Tsubakimoto, Y. Fujimoto, H. Kubomura, S. Matsuoka, T. Ikegawa, Y. Suzuki, and N. Tsuchiya, Journal of Physics: Conference Series 2008, 112, 032058.

[30] H. Furuse, J. Kawanaka, K. Takeshita, N. Miyanaga, T. Saiki, K. Imasaki, M. Fujita, 
and S. Ishii, Optics Letters. 2009, 34, 3439-3441.

[31] R. Yasuhara, H. Furuse, A. Iwamoto, J. Kawanaka, and T. Yanagitani, Optics Express. 2012, 20, 29531-29539.

[32] R. Boulesteix, A. Maitre, J.-F. Baumard, C. Sallé, and Y. Rabinovitch, Optical Materials 2009, 31, 711-715.

[33] H. Yang, X. Qin, J. Zhang, J. Ma, D. Tang, S. Wang, and Q. Zhang, Optical Materials 2012, 34, 940-943.

[34] S. M. Kaczmarek, T. Tsuboi, M. Ito, G. Boulon, and G. Leniec, Journal of Physics: Condensed Matter. 2005, 17, 3771.

[35] X. Wang, Y. Liu, P. Zhao, Z. Guo, Y. Li, and S. Qu, Journal of Applied Physics. 2015, 117, 153104.

[36] D. Sumida, and T. Fan, Optics Letters. 1994, 19, 1343-1345.

[37] L. D. DeLoach, S. A. Payne, L. Chase, L. K. Smith, W. L. Kway, and W. F. Krupke, IEEE Journal of Quantum Electronics 1993, 29, 1179-1191.

[38] E. Perevezentsev, I. Mukhin, O. Vadimova, O. Palashov, E. Khazanov, D. Luo, J. Zhang, and D. Tang, Physica Status Solidi (a) 2013, 210, 1232-1234. 



\section{Chapter 5*}

\section{Development of Yb:GdYAG Mixed Garnet Laser Ceramics}

In ultra short pulsed laser system, in order to achieve adequate bandwidth, the possible way is to select the proper doping elements in laser crystal or to use the disordered crystal as host material. In this chapter, Yb doped GdYAG mixed garnet laser ceramics is successfully fabricated by applying the fabrication method for Yb:YAG ceramics. By using the obtained Yb:GdYAG ceramics as the active laser gain medium, CW and ultra short pulse mode-locked laser is also successfully demonstrated.

*This section published substantially as: Luo et al. "Diode pumped and mode-locked Yb:GdYAG ceramic lasers." Laser Physics Letters, 2011, 8, 719-722. 


\subsection{Introduction}

In laser crystals such as rare-earth doped alumimium garnets, by substitution of rare-earth elements or the ions in the laser host material, spectroscopic properties could be changed. Kimura et al. has studied the rare-earth distribution behavior and lattice parameter changes on the gallium and alumina garnet single crystals [1]. For example, in neodymium $(\mathrm{Nd})$ doped crystal, because the gain bandwidth is relatively narrow, so it is difficult to generate high efficient Q-switched laser. In order to obtain wide bandwidth, one method is to use the disordered solid solutions as the crystal matrix rather than simple crystal hosts [2-10], such as yttrium scandium aluminum garnet (YSAG), gadolinium aluminum gallium garnet, (GAGG), yttrium gallium aluminum garnet (YGAG), and yttrium scandium gallium garnet (YSGG) [11-14]. Among these disordered crystals, gadolinium yttrium aluminum garnet (GdYAG) belongs to the group of oxide compounds crystallizing in garnet structure. This compound was first reported by B. Cockayne et al. in 1968 as single crystal for laser purposes [15]. The end compound gadolinium aluminum garnet ( $\mathrm{GdAG}$ ) was also obtained from the melt by Manabe et al. in 1971 [16]. GdYAG is a mixed oxide compounds formed in distorted garnet structure. In this mixed garnets, the $\mathrm{Y}^{3+}$ ions $(0.92 \AA)$ at the dodecahedron position are partially substituted by $\mathrm{Gd}^{3+}$ ions $(0.97 \AA)$. As $\mathrm{Gd}^{3+}$ ions are larger than $\mathrm{Y}^{3+}$ ions, it results in distortion of the garnet structure. Compared with YAG, in this GdYAG mixed garnets, a locally variable crystal field around the dopant ions is observed. The emission linewidths of the electronic transitions for the $\mathrm{Nd}^{3+}$ are found to be broader than that in ordered crystals [17].

It has also been reported of $\mathrm{Gd}^{3+}$ substitution on the $\mathrm{Y}^{3+}$ sites will red shift the light emission band to longer wavelength in the Cerium doped GdYAG ceramics phosphor for white light emitting diodes applications [18-19]. Park et al. also studied the sintering temperature effect on structural and luminescence properties of Cerium doped GdYAG phosphors [20]. The scintillation properties of cerium doped GdYAG single crystals grown by micro-pulling-down method was studied by Kamada et al. in 2012 [21]. Walsh et al. have also reported the wavelength tuning properties of Nd-doped YAG, GYAG and 
YGAG mixed single crystal [22]. CW laser properties were reported by Di et al. stating increased pump efficiencies with replacing $\mathrm{Y}^{3+}$ ions with large $\mathrm{Gd}^{3+}$ ions [23]. In 2011, Zhou et al. reported that broad gain spectra could be obtained in the $\mathrm{Nd}: \mathrm{Gd}_{0.5} \mathrm{Y}_{2.5} \mathrm{Al}_{5} \mathrm{O}_{12}$ single crystals [24]. The crystal sample showed broad absorption and emission bandwidth compared to the Nd:YAG single crystal. $\mathrm{CW}$ and passively Q-switched laser operation was also demonstrated. Yanagida et al. have reported the fabrication of the Cerium doped $(\mathrm{YGd})_{3} \mathrm{Al}_{5} \mathrm{O}_{12}$ ceramics [25], achieving a higher stopping power to gamma-rays in its scintillation applications [26]. However there's no reports focused on the Yb doped GdYAG mixed garnet ceramics. Based on the fabrication and characterization method established in the previous chapter, we have now successfully fabricated a new type of ytterbium-doped yttrium gadolinium aluminum garnet (Yb:GdYAG) ceramics. The CW and passive mode-locking operations of the mixed ceramic have also been experimentally investigated.

\subsection{Experimental methods}

\subsubsection{Fabrication and characterization of $\mathrm{Yb}$ :GdYAG laser ceramics}

The Yb:GdYAG ceramics were fabricated in-house using a solid-state reactive sintering method under vacuum condition. The detailed fabrication process was similar to that used for fabricating the $\mathrm{Yb}: \mathrm{YAG}$ ceramics in the previous chapter. The raw materials used were high-purity (>99.99\%) $\mathrm{Al}_{2} \mathrm{O}_{3}, \mathrm{Y}_{2} \mathrm{O}_{3}, \mathrm{Yb}_{2} \mathrm{O}_{3}$, and $\mathrm{Gd}_{2} \mathrm{O}_{3}$ powders. The respective raw powders were carefully weighed to form the chemical stoichiometry composition of 5.0 at.\% $\mathrm{Yb}: \mathrm{Gd}_{0.5} \mathrm{Y}_{2.5} \mathrm{Al}_{5} \mathrm{O}_{12}$. The raw materials were then gone through similar ball milling, calcinations, CIP pressing, vacuum sintering, annealing and final polishing and cutting process to obtain the final finished sample. UV-VIS-NIR spectrophotometer (Carry 5000 Spectrophotometer, Varian, USA) and spectrofluorometer (Fluorolog-3, Jobin Yvon, Edison, USA) were used to measure the sample's room temperature absorption and emission spectra. CW and mode locked laser system were constructed to demonstrate samples' laser performances. 


\subsection{Results and discussion}

\subsubsection{Spectroscopic study}

The sintered Yb:GdYAG ceramics were round pellets with diameter of 16.0 and $3.5 \mathrm{~mm}$ in thickness. The room temperature absorption and emission spectra of the Yb:GdYAG ceramics are shown in Figure 5.1. The sample has the absorption peak at $940 \mathrm{~nm}$ wavelength with an absorption coefficient of $4.9 \mathrm{~cm}^{-1}$. Two moderate absorption peaks are located at $915 \mathrm{~nm}$ and $969 \mathrm{~nm}$ respectively, corresponding to the ${ }^{2} \mathrm{~F}_{7 / 2} \rightarrow{ }^{2} \mathrm{~F}_{5 / 2}$ transition of $\mathrm{Yb}^{3+}$ ions. For the normalized emission spectrum, the emission peak is at $1028 \mathrm{~nm}$ with a full width at the half maximum (FWHM) of $11.95 \mathrm{~nm}$. Compared to the FWHM of $11.07 \mathrm{~nm}$ for 5.0 at.\% Yb:YAG ceramic, which was sintered under the same conditions in previous chapter. The emission line was increased by around $1 \mathrm{~nm}$. The broadened emission band is useful for generating ultra short mode locked pulses.

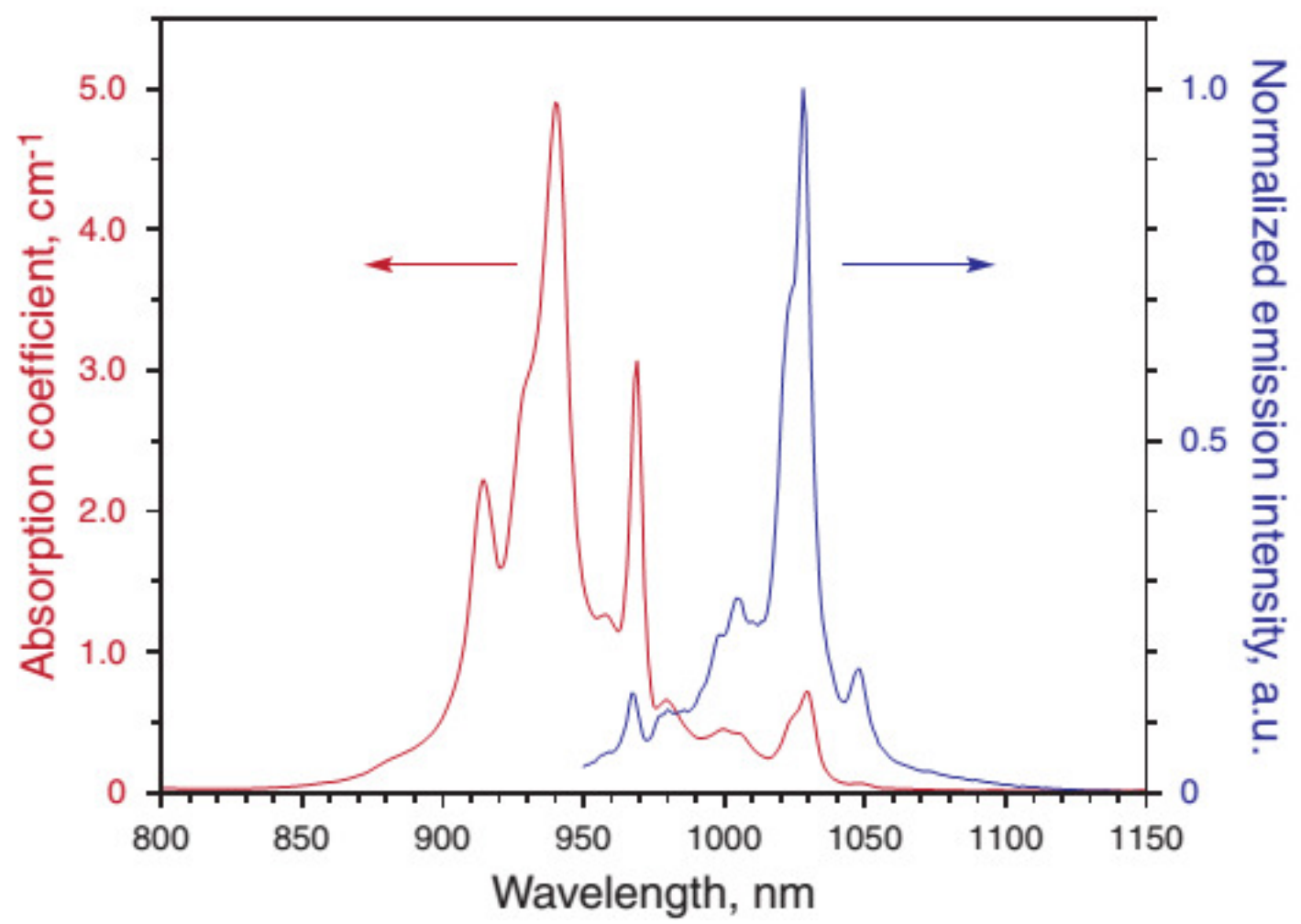

Figure 5.1 The room temperature absorption and normalized emission spectra of a 5.0 at.\% Yb:GdYAG ceramic. 


\subsubsection{CW laser operation study}

The CW laser operation of the ceramic was first investigated. A laser setup as shown in Figure 5.2 was used. The ceramic was pumped by a fiber coupled diode bar at the wavelength of $940 \mathrm{~nm}$. The coupling fiber had a core diameter of $100 \mu \mathrm{m}$ and a numerical aperture (NA) of 0.22. M1 was a high reflection mirror. The Yb:GdYAG ceramic sample was cut into dimensions of $3.0 \times 3.0 \times 3.2 \mathrm{~mm}^{3}$ and coated high transmission (HT) on both faces at the pump and laser wavelengths. The ceramic was wrapped in indium foil and mounted in a copper heat sink whose temperature was maintained at $19^{\circ} \mathrm{C}$ throughout the experiment. Figure 5.3 shows the $\mathrm{CW}$ output power versus the incident pump power of the laser under different output couplings (Toc $=3.2 \%$, $7.5 \%, 10.0 \%$, and $17.0 \%$ ). With the Toc value increasing, the laser output power and the slope efficiency were also increased obviously. The CW oscillation wavelength of the laser was at $1030 \mathrm{~nm}$. A maximum output of $4.27 \mathrm{~W}$ was achieved under $18.61 \mathrm{~W}$ incident pump power with the Toc $=17.0 \%$. The corresponding slope efficiency of the laser is $26.6 \%$. No saturation phenomenon was observed during the testing.

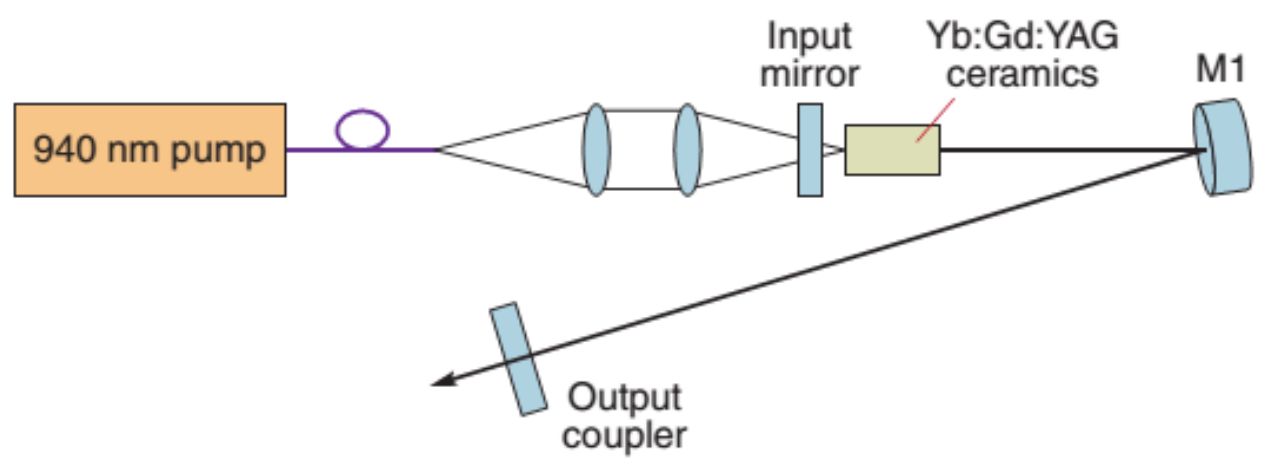

Figure 5.2 The cavity configuration of a Yb:GdYAG ceramic laser in CW operation. 


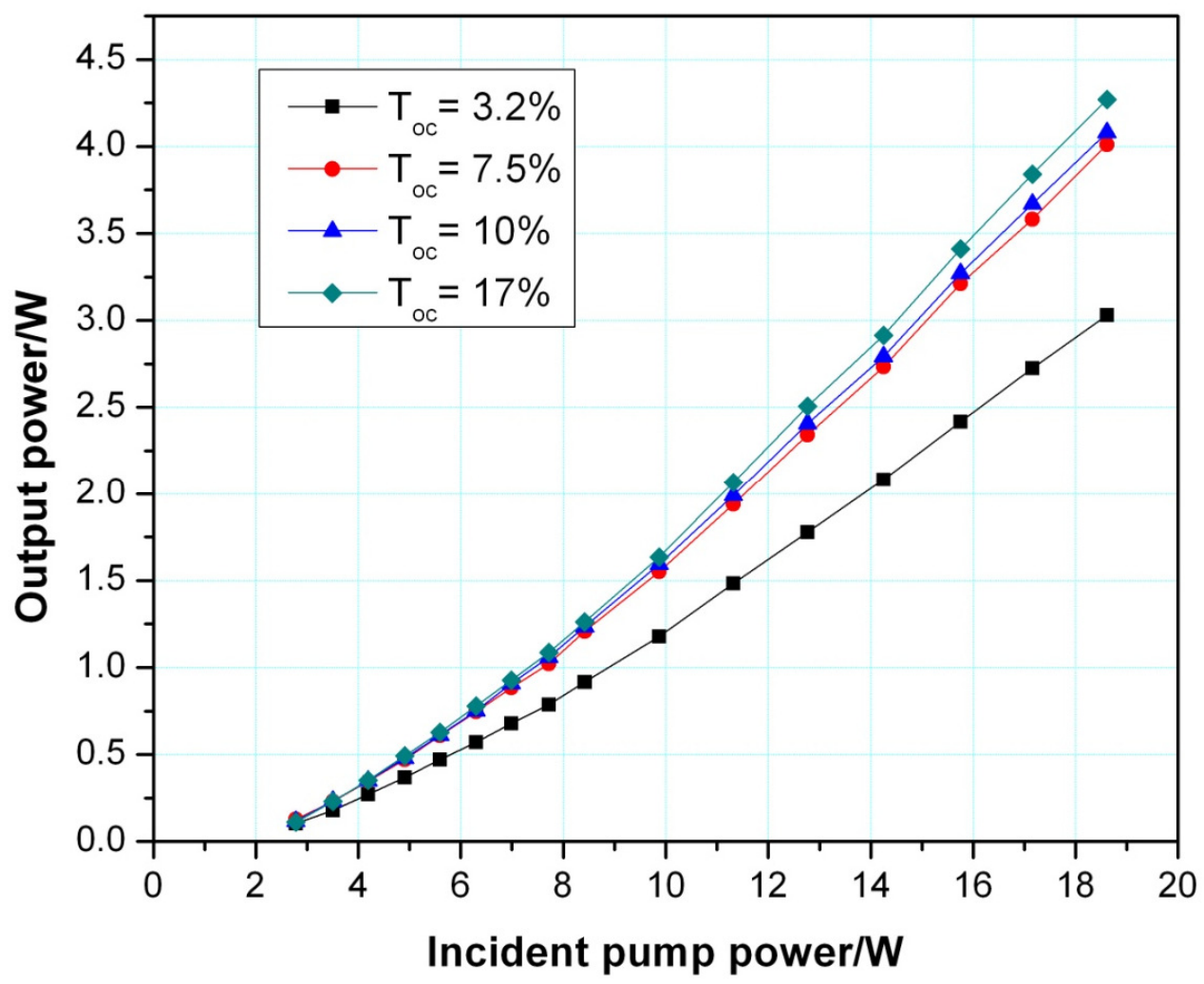

Figure 5.3 Output power versus the incident pump power of a 5.0 at.\% Yb:GdYAG ceramic under $\mathrm{CW}$ operation.

\subsubsection{Mode locked laser performance}

The passive mode-locking of the ceramic laser was then studied using a cavity setup as shown in Figure 5.4. The pump source and ceramic sample used were the same as in the CW operation case. An X-shaped cavity was adopted, where M1 (Roc=300 mm), M2 $(\mathrm{Roc}=500 \mathrm{~mm})$, and M3 (Roc=500 mm) were broadband high reflection coated in the range of $1000 \mathrm{~nm}$ to $1100 \mathrm{~nm}$. A commercial semiconductor saturable absorber mirror (SESAM) (BATOP GmbH) was used to initiate the mode-locking. The SESAM has a modulation depth of $1 \%$, a non-saturable loss of $0.3 \%$ and a relaxation time of $500 \mathrm{fs}$. A fast detector (New Focus 1611, $1 \mathrm{GHz}$ bandwidth) together with a digital oscilloscope (Tektronix DPO 7104, $1 \mathrm{GHz}$ bandwidth) was employed to monitor the mode-locking pulse train. An optical spectrum analyzer (Ando AQ 6315B) was used to measure the 
optical spectrum and a commercial autocorrelator (APE GmbH, Pulsecheck) was used to measure the pulse width.

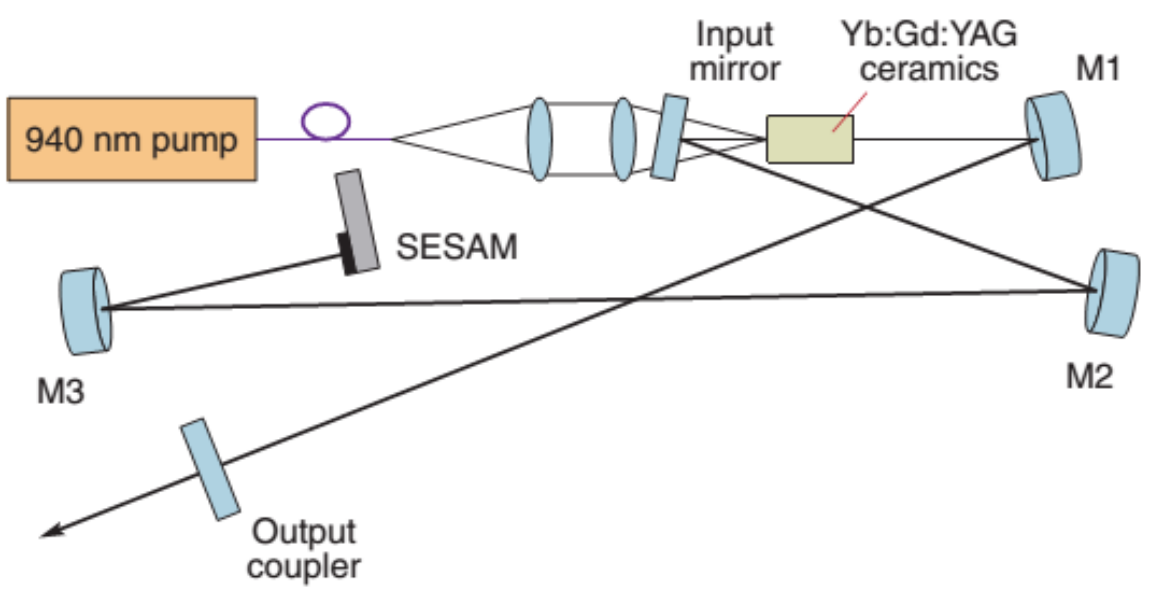

Figure 5.4 The experimental setup for mode-locked Yb:GdYAG ceramic laser.

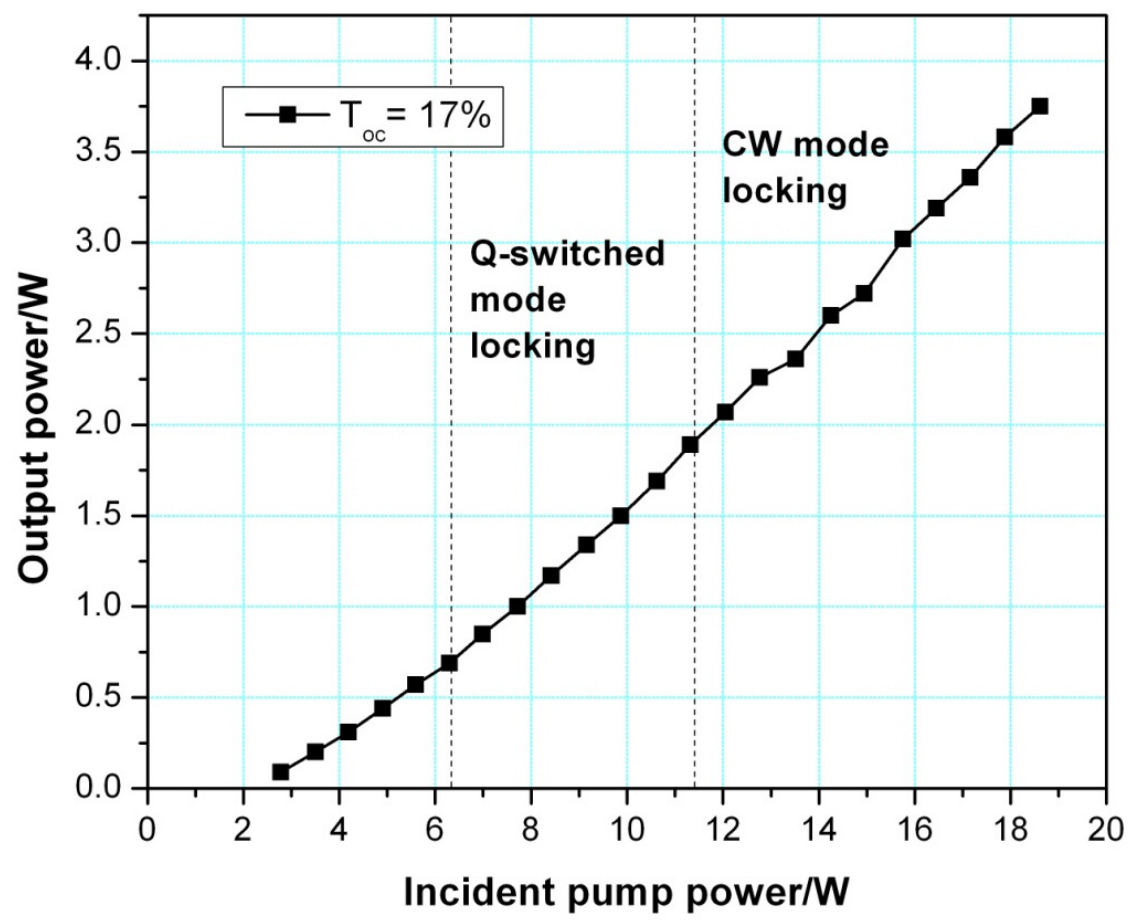

Figure 5.5 The Output power of mode-locked Yb:GdYAG ceramic laser with respect to the incident pump power. 
Figure 5.5 shows the average output power of the mode-locked laser with respect to the incident pump power when $\mathrm{Toc}=17.0 \%$. The laser was in the $\mathrm{CW}$ operation with a threshold incident pump power of $2.78 \mathrm{~W}$. Q-switched mode-locking was observed when the incident pump power increased to $6.3 \mathrm{~W}$. The stable $\mathrm{CW}$ mode-locking was obtained when the incident pump power increased to $11.3 \mathrm{~W}$. A maximum output power of $3.75 \mathrm{~W}$ was obtained when the incident pump power was $18.61 \mathrm{~W}$. The corresponding slope efficiency was $25.8 \%$.

The mode-locked pulse train has a repetition rate of $64 \mathrm{MHz}$. Figure 5.6 shows the autocorrelation trace of the mode-locked pulses. Assuming that the pulses have a Gaussian pulse profile, the mode-locked pulse width is 4.4 ps. The optical spectrum of mode-locked pulse is plotted in Figure 5.7. The pulse is centered at $1030.4 \mathrm{~nm}$ with a FWHM of $0.86 \mathrm{~nm}$. The time-bandwidth product of the mode-locked pulses is about 1.07, indicating that the pulses are slightly chirped.

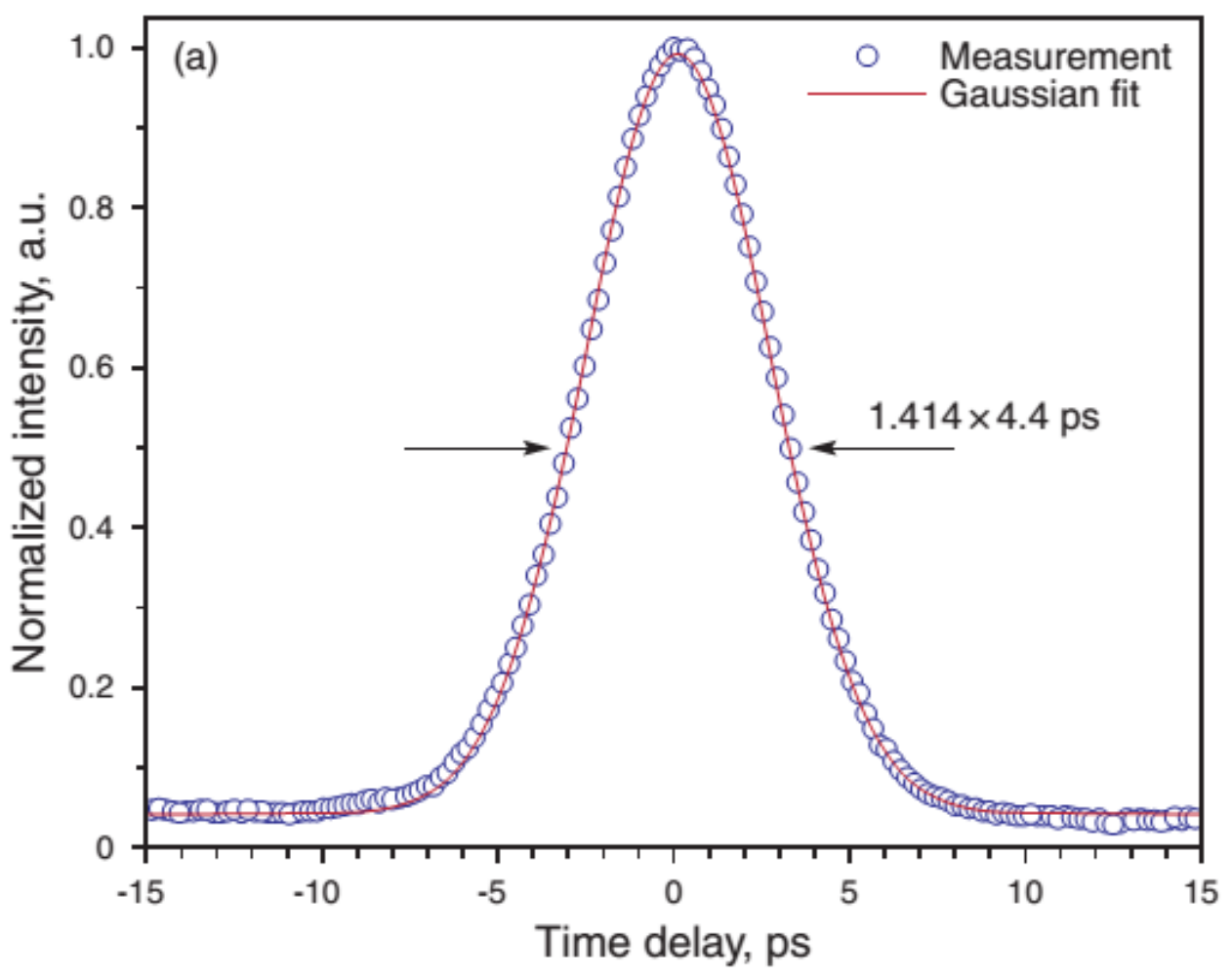

Figure 5.6 Autocorrelation trace of the mode-locked Yb:GdYAG ceramics laser. 


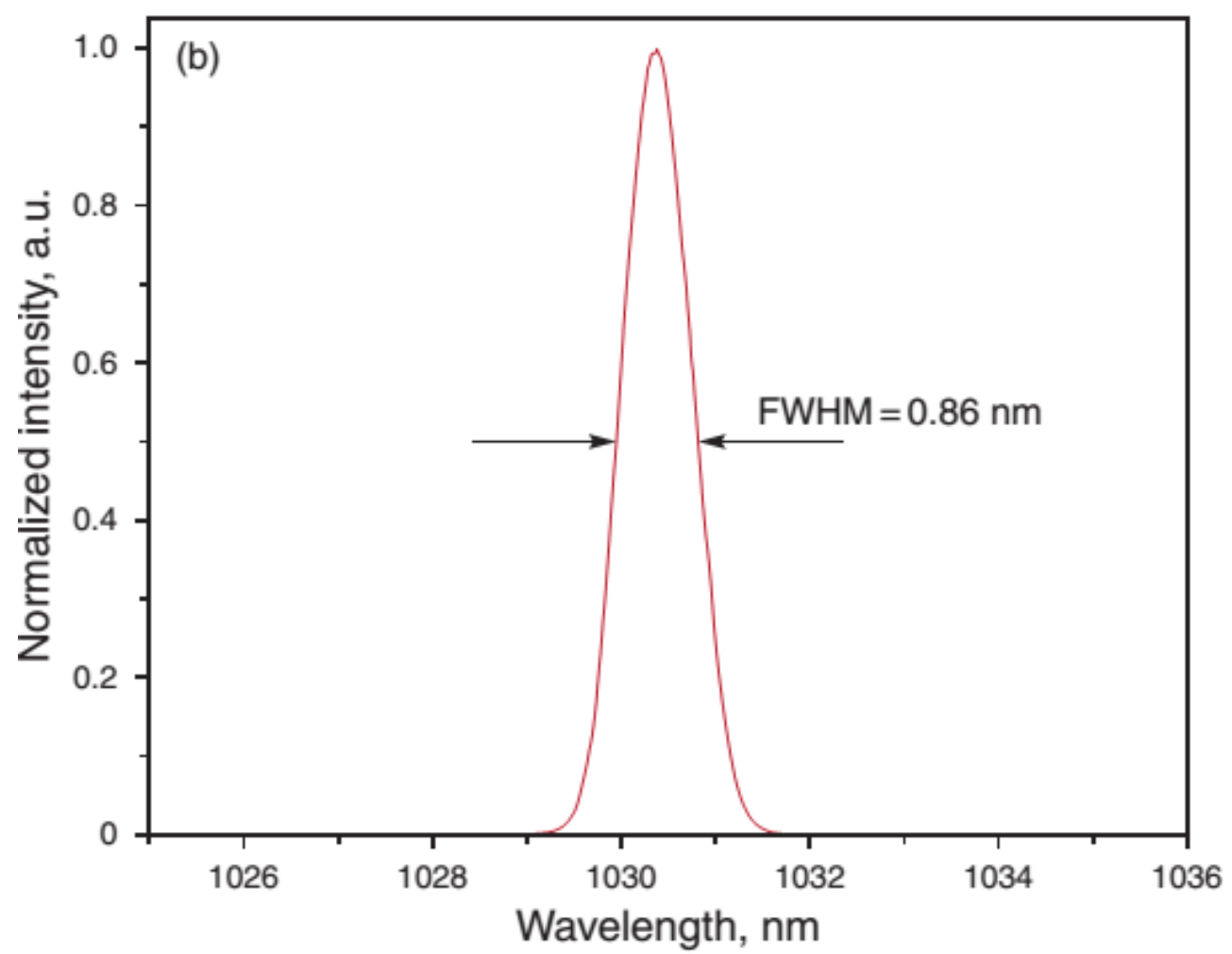

Figure 5.7 Optical spectrum of mode-locked pulse for Yb:GdYAG ceramics laser.

\subsection{Conclusions}

We have successfully fabricated a new type of ytterbium-doped gadolinium yttrium aluminum garnet (Yb:GdYAG) laser ceramic using the solid-state reactive sintering method under vacuum condition. Continuous wave (CW) laser operation of the 5.0 at.\% Yb:GdYAG ceramic laser has achieved $4.27 \mathrm{~W}$ output power with $26.7 \%$ slope efficiency. Passive mode-locking properties of the ceramic were also firstly demonstrated with a semiconductor saturable absorbing mirror (SESAM). Stable mode-locked pulses with 4.4 ps pulse width and a maximum output power of $3.75 \mathrm{~W}$ at the wavelength of $1030.4 \mathrm{~nm}$ have been generated. Researchers have also experimentally studied the mode locked laser with a near isotropic cavity by using this Yb:GdYAG ceramic samples provided by the author. They have shown that the laser can simultaneously mode lock in the two orthogonal principal polarization directions of the cavity, which was the first experimental demonstration of the antiphase dynamics of mode locked lasers [27]. It is an 
implication that more applications in solid state laser could be realized by using laser ceramics.

\section{References}

[1] H. Kimura, T. Numazawa, and M. Sato, Journal of Materials Science Letters. 1994, 13, 1164-1168.

[2] A. A. Kaminskii, Laser crystals: their physics and properties, Springer, 2013.

[3] M. Voda, F. Jaque, J. G. Solé, and A. Kaminskii, Chemical Physics Letters 1993, $213,84-88$.

[4] Z. Pan, H. Cong, H. Yu, L. Tian, H. Yuan, H. Cai, H. Zhang, H. Huang, J. Wang, and Q. Wang, Optics Express 2013, 21, 6091-6100.

[5] G. Xie, D. Tang, W. Tan, H. Luo, H. Zhang, H. Yu, and J. Wang, Optics letters 2009, 34, 103-105.

[6] H. Eichler, D. Ashkenasi, H. Jian, and A. Kaminskii, Physica Status Solidi (a) 1994, $146,833-841$.

[7] H. Yu, H. Zhang, Z. Wang, J. Wang, Y. Yu, Z. Shi, X. Zhang, and M. Jiang, Optics letters 2009, 34, 151-153.

[8] G. Xie, L. Qian, P. Yuan, D. Tang, W. Tan, H. Yu, H. Zhang, and J. Wang, Laser Physics Letters 2010, 7, 483-486.

[9] G. Xie, D. Tang, W. Tan, H. Luo, S. Guo, H. Yu, and H. Zhang, Applied Physics B 2009, 95, 691-695.

[10] H. Zhang, J. Liu, J. Wang, J. Fan, X. Tao, X. Mateos, V. Petrov, and M. Jiang, Optics express 2007, 15, 9464-9469.

[11] T. H. Allik, C. A. Morrison, J. B. Gruber, and M. R. Kokta, Physical Review B 1990, 41, 21.

[12] J. Zhang, X. Tao, C. Dong, Z. Jia, H. Yu, Y. Zhang, Y. Zhi, and M. Jiang, Laser Physics Letters 2009, 6, 355-358.

[13] B. M. Walsh, N. P. Barnes, R. L. Hutcheson, R. W. Equall, and B. Di Bartolo, Journal of the Optical Society of America B 1998, 15, 2794-2801. 
[14] S. Wang, K. Wu, Y. Wang, H. Yu, H. Zhang, X. Tian, Q. Dai, and J. Liu, Optics express 2013, 21, 16305-16310.

[15] B. Cockayne, D. Gasson, D. Findlay, D. Goodwin, and R. Clay, Journal of Physics and Chemistry of Solids 1968, 29, 905-910.

[16] T. Manabe, and K. Egashira, Materials Research Bulletin 1971, 6, 1167-1174.

[17] H. Okada, M. Tanaka, H. Kiriyama, Y. Nakai, Y. Ochi, A. Sugiyama, H. Daido, T. Kimura, T. Yanagitani, and H. Yagi, Optics letters 2010, 35, 3048-3050.

[18] S. Nishiura, S. Tanabe, K. Fujioka, and Y. Fujimoto, SPIE OPTO 2011, 793404.

[19] S. Nishiura, S. Tanabe, K. Fujioka, and Y. Fujimoto, IOP Conference Series: Materials Science and Engineering 2011, 18, 102005.

[20] J. Y. Park, H. C. Jung, G. S. R. Raju, B. K. Moon, J. H. Jeong, S.-M. Son, and J. H. Kim, Optical Materials 2009, 32, 293-296.

[21] K. Kamada, T. Yanagida, J. Pejchal, M. Nikl, and A. Yoshikawa, Physica Status Solidi (c) 2012, 9, 2292-2295.

[22] B. M. Walsh, N. P. Barnes, R. Hetcheson, R. W. Equall, and B. Di Bartolo, Lasers and Electro-Optics 1999, 292-293.

[23] J. Di, X. Xu, D. Li, D. Zhou, F. Wu, Z. Zhao, J. Xu, and D. Tang, Laser Physics 2011, 21, 1742-1744.

[24] D. Zhou, X. Xu, J. Meng, D. Li, J. Di, C. Xia, F. Wu, and J. Xu, Laser Physics Letters 2011, 8, 201-204.

[25] T. Yanagida, A. Yoshikawa, M. Kawaharada, T. Itoh, and F. Saito, 2007 IEEE Nuclear Science Symposium Conference Record 2007, 2, 1343-1346.

[26] T. Yanagida, T. Itoh, H. Takahashi, S. Hirakuri, M. Kokubun, K. Makishima, M. Sato, T. Enoto, T. Yanagitani, and H. Yagi, Nuclear Instruments and Methods in Physics Research Section A: Accelerators, Spectrometers, Detectors and Associated Equipment. 2007, 579, 23-26.

[27] C. Xu, D. Tang, H. Zhu, and J. Zhang, Laser Physics Letters 2013, 10, 095702. 



\section{Chapter 6*}

\section{Development of Yb:YLuAG Laser Ceramics}

Lutetium aluminium garnet (LuAG) is another new type of laser host material. Similar with YAG, it has cubic crystal structure, with a relatively higher density and melting point. This host material has the smallest lattice constant of the rare earth garnets. The crystal field in LuAG yields a narrower linewidths and higher absorption and emission cross-sections. LuAG has a higher thermal conductivity, which makes it another potential candidate for Yb host material in high power solid state lasers. In this chapter, Yb doped YLuAG laser ceramics are successfully fabricated by applying the previously established fabrication method for YAG ceramics. By using the obtained Yb:YLuAG ceramics as the active laser gain medium, $C W$ and ultra short pulse mode-locked laser is also successfully demonstrated.

*This section published substantially as: Luo et al. "Yb:LuAG laser ceramics: a promising high power laser gain medium." Optical Materials Express. 2012, 2, 1425-1431. and Luo et al. "Modelocked Yb:LuAG ceramics laser." Physica Status Solidi (c). 2013, 10, 967-968. 


\subsection{Introduction}

In high power solid state laser operation, there is unavoidably a large amount of heat generation. If not be efficiently dissipated, it could cause severe thermal problems, even lead to permanent laser gain medium damage. Therefore, use a laser gain material with good thermal conductivity becomes especially important for practical high power laser applications [1-4]. As discussed in the previous chapter, YAG materials are widely used as the laser host material because of its good thermal properties. However, it was found that as the $\mathrm{Yb}$ doping concentration is increased, the thermal conductivity of $\mathrm{Yb}$ :YAG drops significantly $[5,6]$. It affects severely the high power performance of the lasers. To solve the problem, solid solution of $(\mathrm{LuY})_{3} \mathrm{Al}_{5} \mathrm{O}_{12}$ (lutetium yttrium aluminium garnet) was proposed as an alternate laser host material. The growth of solid solution LuYAG single crystal has also been reported by Kuwano et al. previously [7].

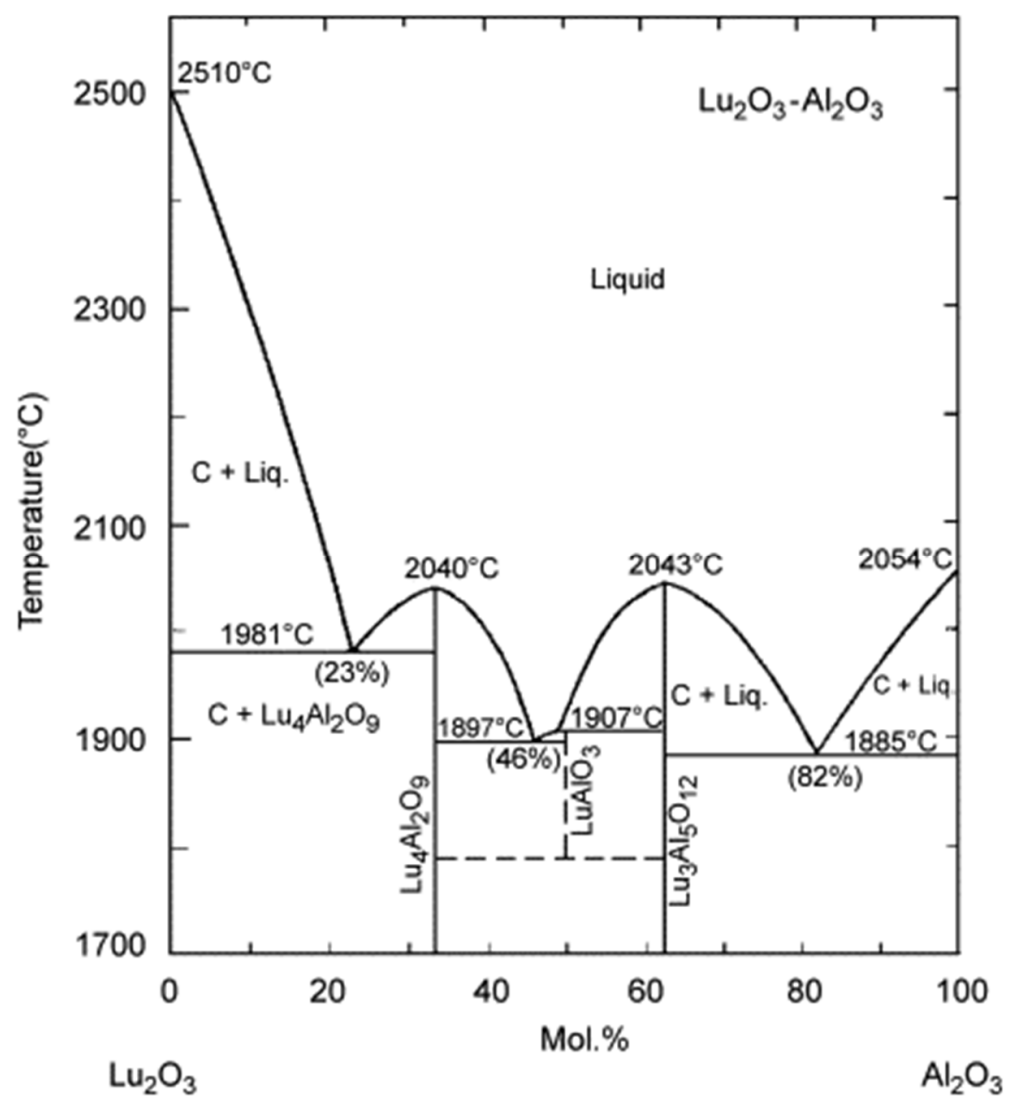

Figure 6.1 $\mathrm{Lu}_{2} \mathrm{O}_{3}-\mathrm{Al}_{2} \mathrm{O}_{3}$ phase diagram. 
In the LuYAG garnet system, the end compound $\mathrm{Lu}_{3} \mathrm{Al}_{5} \mathrm{O}_{12}$ lutetium aluminium garnet (LuAG) has a high density of $6.72 \mathrm{~g} / \mathrm{cm}^{3}$, a high thermal conductivity of $9.6 \mathrm{w} / \mathrm{m} \cdot \mathrm{K}$ and a cubic structure that is optically isotropic. The $\mathrm{Lu}_{2} \mathrm{O}_{3}-\mathrm{Al}_{2} \mathrm{O}_{3}$ system has been extensively studied [8-10]. Its phase diagram is shown in Figure 6.1. Various LuAG crystals lasers have been reported over the years [11-15]. Scintillation properties and applications have also been studied for LuAG crystals [16-19].

In $\mathrm{Yb}$ doped LuAG crystal, the atomic mass and ion radius of $\mathrm{Lu}$ ion $(174.97,100.1 \mathrm{pm})$ are close to those of the $\mathrm{Yb}$ ion $(173.04,100.8 \mathrm{pm})$ rather than the $\mathrm{Y}$ ion $(88.9059,104$ $\mathrm{pm})$. Therefore at high $\mathrm{Yb}$ doping concentrations, the smaller mass difference between the host cation and the dopant cation would cause less phonon scattering, which is the main reason for the drop in thermal conductivity [20]. Although the thermal conductivity of undoped LuAG is slightly smaller than that of the YAG, the thermal conductivity of $\mathrm{Yb}: \mathrm{LuAG}$ becomes well above $\mathrm{Yb}$ :YAG when $\mathrm{Yb}$ doping concentration is increased to be larger than 5\%. The comparison of thermal conductivity between Yb:YAG and $\mathrm{Yb}: \mathrm{LuAG}$ as a function of $\mathrm{Yb}$ doping concentration is plotted in Figure 6.2 [20].

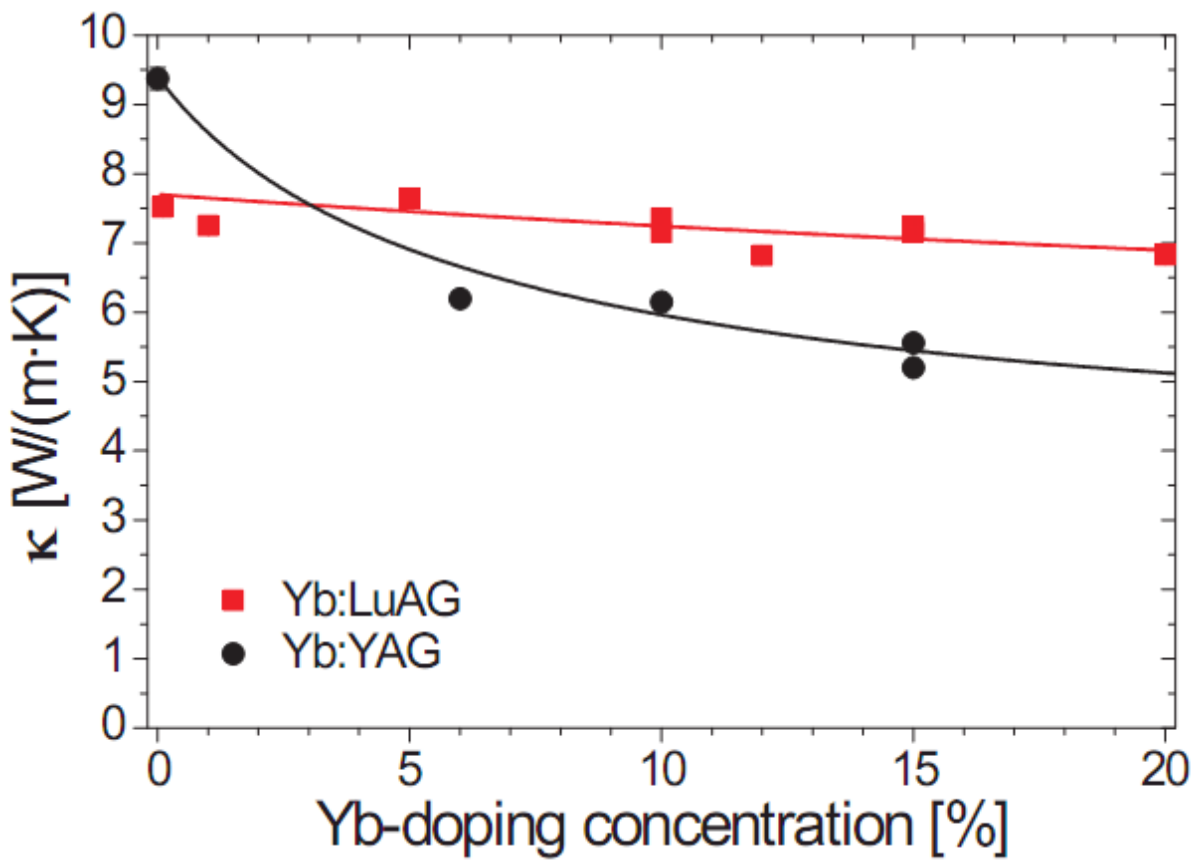

Figure 6.2 Thermal conductivity of $\mathrm{Yb}: \mathrm{YAG}$ and $\mathrm{Yb}: \mathrm{LuAG}$ as a function of $\mathrm{Yb}$ doping concentration. 
This advantageous thermal property of Yb:LuAG has also attracted many researchers' attention. Various Yb:LuAG crystal have been utilized for laser applications. Sumida et al. reported the first room temperature diode pumped $\mathrm{Yb}: \mathrm{LuAG}$ single crystal laser in 1995 [21]. Brenier et al. reported growth, spectroscopic, and laser properties of the $\mathrm{Yb}: \mathrm{LuAG}$ crystal in 2006 [22]. Beil et al. reported a $5 \mathrm{~kW}$ Yb:LuAG thin disk laser with optical-to-optical efficiency of over 60\% [5]. The first Q-switched Yb:LuAG microchip laser was reported in 2007 [23]. He et al. have also achieved mode-locking of a Yb:LuAG single crystal laser with $~ 7.6$ ps pulse width [24]. High efficient microchip $\mathrm{Yb}: \mathrm{LuAG}$ laser was also reported in 2010 [25].

There are a few reports focused on the synthesis of LuAG ceramics powder precursors previously $[26,27]$. However, there's only very few reports on LuAG ceramics lasers [28-31], since the fabrication of high optical quality LuAG ceramics still remains a challenging task. In this chapter, we apply the fabrication method for Yb:YAG ceramics to obtain the transparent Yb:YLuAG laser ceramics. LuAG ceramics with Yb doping concentrations from $5 \%$ to $20 \%$ were successfully fabricated by using solid-state reactive sintering method. High power high efficient lasing operation of the fabricated $\mathrm{Yb}: \mathrm{LuAG}$ ceramics was experimentally demonstrated.

\subsection{Experimental methods}

\subsubsection{Fabrication of $\mathrm{Yb}: Y L u A G$ laser ceramics}

The raw materials used for the $\mathrm{Yb}$ :YLuAG ceramics fabrication were high purity $\mathrm{Yb}_{2} \mathrm{O}_{3}$, $\mathrm{Lu}_{2} \mathrm{O}_{3}, \mathrm{Y}_{2} \mathrm{O}_{3}$ and $\mathrm{Al}_{2} \mathrm{O}_{3}$ powders. The fabrication process is similar to that of $\mathrm{Yb}: \mathrm{YAG}$ ceramics, as discussed in the previous chapter. The raw powders will go through precise weighing, high energy ball milling, slurry drying and sieving, cold press and CIP shaping, vacuum sintering, annealing and polishing process to obtain the final dense ceramic samples. The YLuAG ceramics were fabricated by adjusting the chemical stoichiometry composition. The $\mathrm{Yb}$ doping concentration is fixed at 5 at.\%. The chemical formula is as follows: $\mathrm{Y}_{3} \mathrm{Al}_{5} \mathrm{O}_{12}, \quad\left(\mathrm{Y}_{0.67} \mathrm{Lu}_{0.33}\right)_{3} \mathrm{Al}_{5} \mathrm{O}_{12}, \quad\left(\mathrm{Y}_{0.5} \mathrm{Lu}_{0.5}\right)_{3} \mathrm{Al}_{5} \mathrm{O}_{12}, \quad\left(\mathrm{Y}_{0.33} \mathrm{Lu}_{0.67}\right)_{3} \mathrm{Al}_{5} \mathrm{O}_{12}$ and 
$\mathrm{Lu}_{3} \mathrm{Al}_{5} \mathrm{O}_{12}$ for the five YLuAG ceramic compounds respectively. Figure 6.3 shows the photo of fabricated $\mathrm{Yb}$ :YLuAG ceramics sintered at $1840^{\circ} \mathrm{C}$.

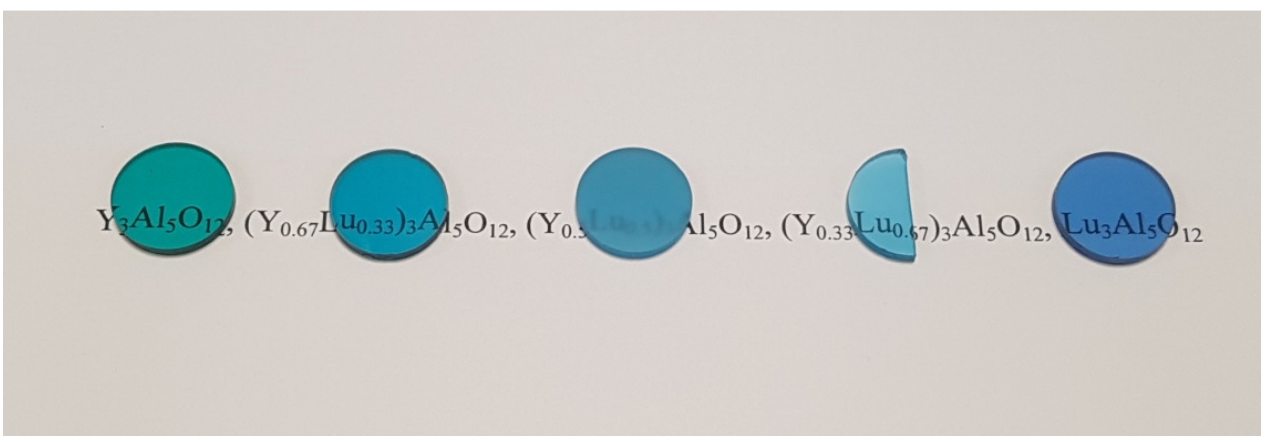

Figure 6.3 In-house fabricated Yb:YLuAG ceramics.

The end compound of LuAG garnet ceramics with different $\mathrm{Yb}$ doping was also fabricated. Photographs of representative $\mathrm{Yb}: \mathrm{LuAG}$ ceramic samples are shown in Figure 6.4 with different $\mathrm{Yb}$ doping. The samples were sintered at $1840^{\circ} \mathrm{C}$ for 8 hours. The samples appear blue in colour before annealing. The colour becomes darker with higher $\mathrm{Yb}$ doping concentration. The colour disappears complete after annealing process. After polishing, all samples appear transparent as shown in the photo, the letters behind them are clearly visible.

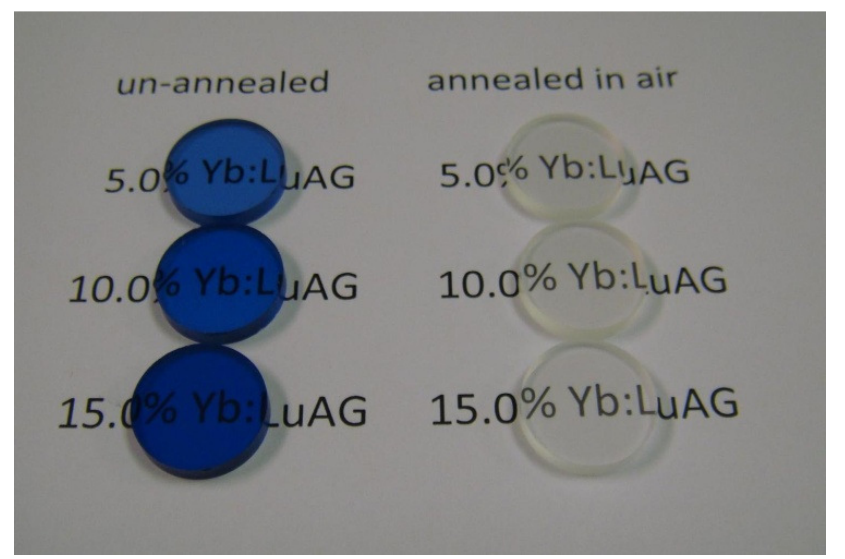

Figure 6.4 Yb:LuAG ceramic samples sintered at $1840^{\circ} \mathrm{C}$ for $8 \mathrm{~h}$ with different doping concentrations. 


\subsubsection{Characterization of Yb:YLuAG laser ceramic}

The raw powders and samples' microstructure were inspected by SEM 6360. XRD (Bruker D8) was used to identify sample phase. Transmittance properties of the samples were measured by spectrophotometer and the sample's fluorescent properties were measured by spectrofluorometer. Solid state laser systems were built to test the sample's laser performances.

\subsection{Results and discussion}

\subsubsection{Phase and microstructure study}

Figure 6.5 shows the XRD pattern of fabricated YLuAG ceramics. All the peaks of the samples are well indexed to the cubic garnet structure of YAG and LuAG. There are slightly shifts of the peaks from YAG base to LuAG base, which is due to the smaller lattice constant for LuAG compared with YAG. The density LuAG ceramics were measured to be around $6.55 \mathrm{~g} / \mathrm{cm}^{3}$. The measured value of LuAG ceramics is comparable with that of LuAG singly crystal reported in other literatures [7].

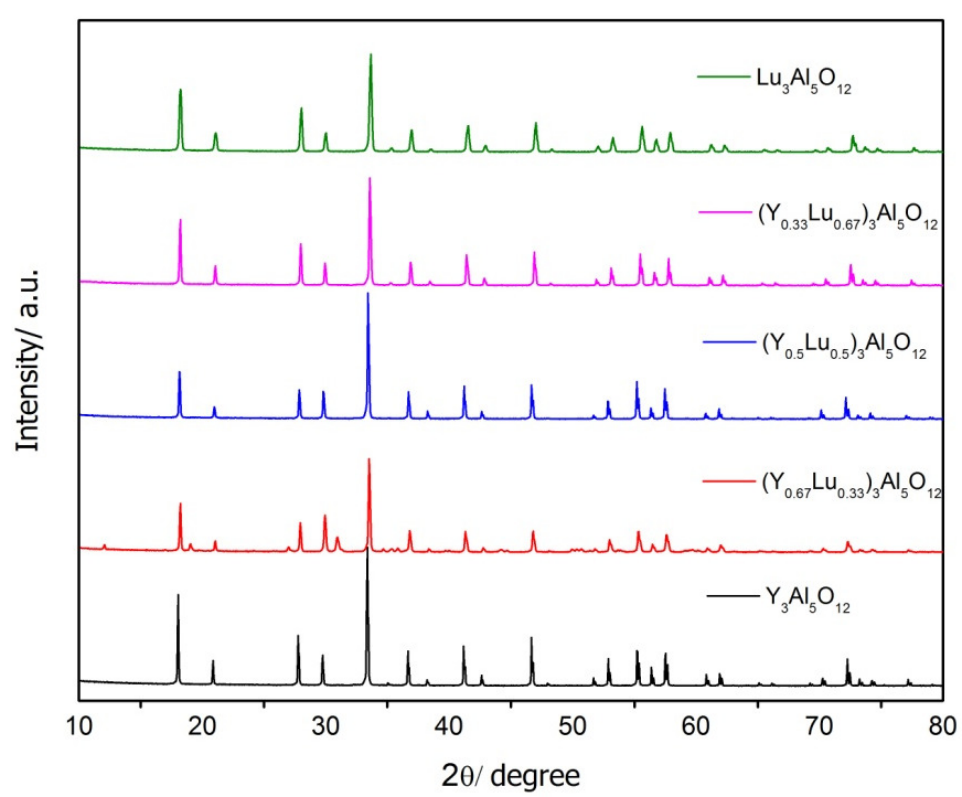

Figure 6.5 XRD patterns of YLuAG ceramics. 
The samples were thermally etched in air before their surface morphology were inspected by using SEM. Surface images of the LuAG ceramics samples with different doping $\mathrm{Yb}$ concentrations are displayed in Figure 6.6. Grain boundaries are clearly observed. The grain size does not vary too much with doping concentration. The average grain size is about $10 \mu \mathrm{m}$. A few of the intraganular pores can be found inside some of the grains. These pores would be light scattering centers and affect the optical quality of the samples.
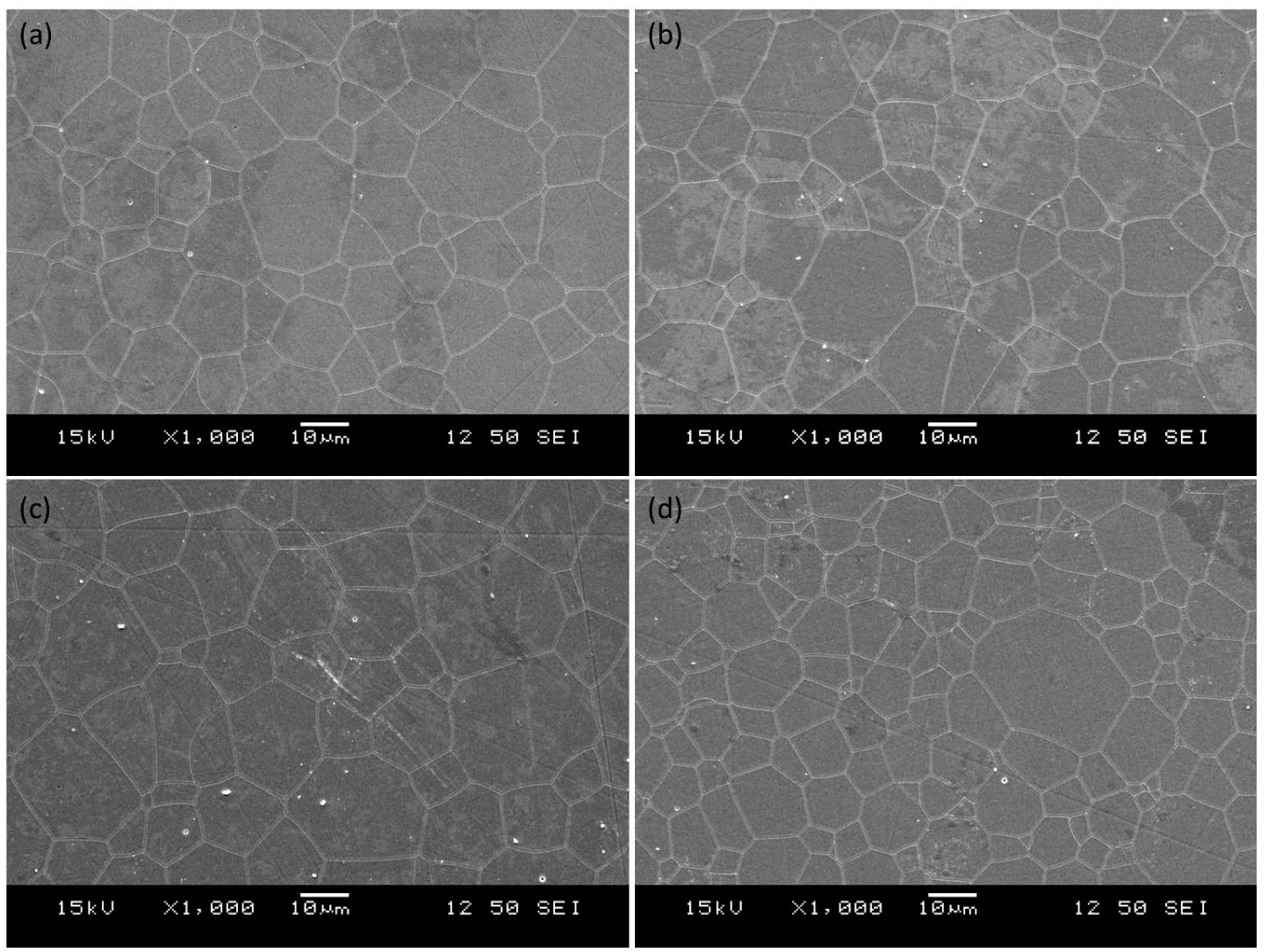

Figure 6.6 Surface SEM images of the Yb:LuAG ceramics sintered at $1840^{\circ} \mathrm{C}$ for $8 \mathrm{~h}$ : (a) 5 at.\%, (b) 10 at. \%, (c) 15 at.\% and (d) 20 at.\% doping concentration.

\subsubsection{Spectroscopic study}

Figure 6.7 displays the room-temperature absorption spectra of the as fabricated $\mathrm{Yb}: \mathrm{LuAG}$ ceramic samples. Absorption spectra of the $\mathrm{Yb}: \mathrm{LuAG}$ ceramics are similar in shape to those of the Yb:YAG ceramics. The highest absorption peak is at $968.4 \mathrm{~nm}$. For 
the samples with $5 \%, 10 \%$ and $15 \% \mathrm{Yb}$ doping concentration the peak absorption coefficients were measured to be $6.4 \mathrm{~cm}^{-1}, 12.2 \mathrm{~cm}^{-1}$ and $16.3 \mathrm{~cm}^{-1}$, respectively. However, this absorption peak is very sharp, with a full width at half maximum (FWHM) of $2.5 \mathrm{~nm}$. It is not very practical to use pump source at this wavelength, since the slight wavelength shift will result in lower absorption efficiency. There is also a broad absorption band at $940 \mathrm{~nm}$ that has double peaks, centered at $935.6 \mathrm{~nm}$ and $939.6 \mathrm{~nm}$, respectively. The two peaks have nearly the same absorption coefficient. The FWHM of this broad absorption band is $21.6 \mathrm{~nm}$, which favors direct diode pumping with InGaAs laser diodes. The absorption cross-section at $939 \mathrm{~nm}$ and $968 \mathrm{~nm}$ absorption peaks is calculated to be $0.73 \times 10^{-20} \mathrm{~cm}^{2}$ and $0.91 \times 10^{-20} \mathrm{~cm}^{2}$, respectively. In addition, an absorption peak at $1030 \mathrm{~nm}$ is also observable on the spectra. The above measured results coincide with those of $\mathrm{Yb}: \mathrm{LuAG}$ single crystals.

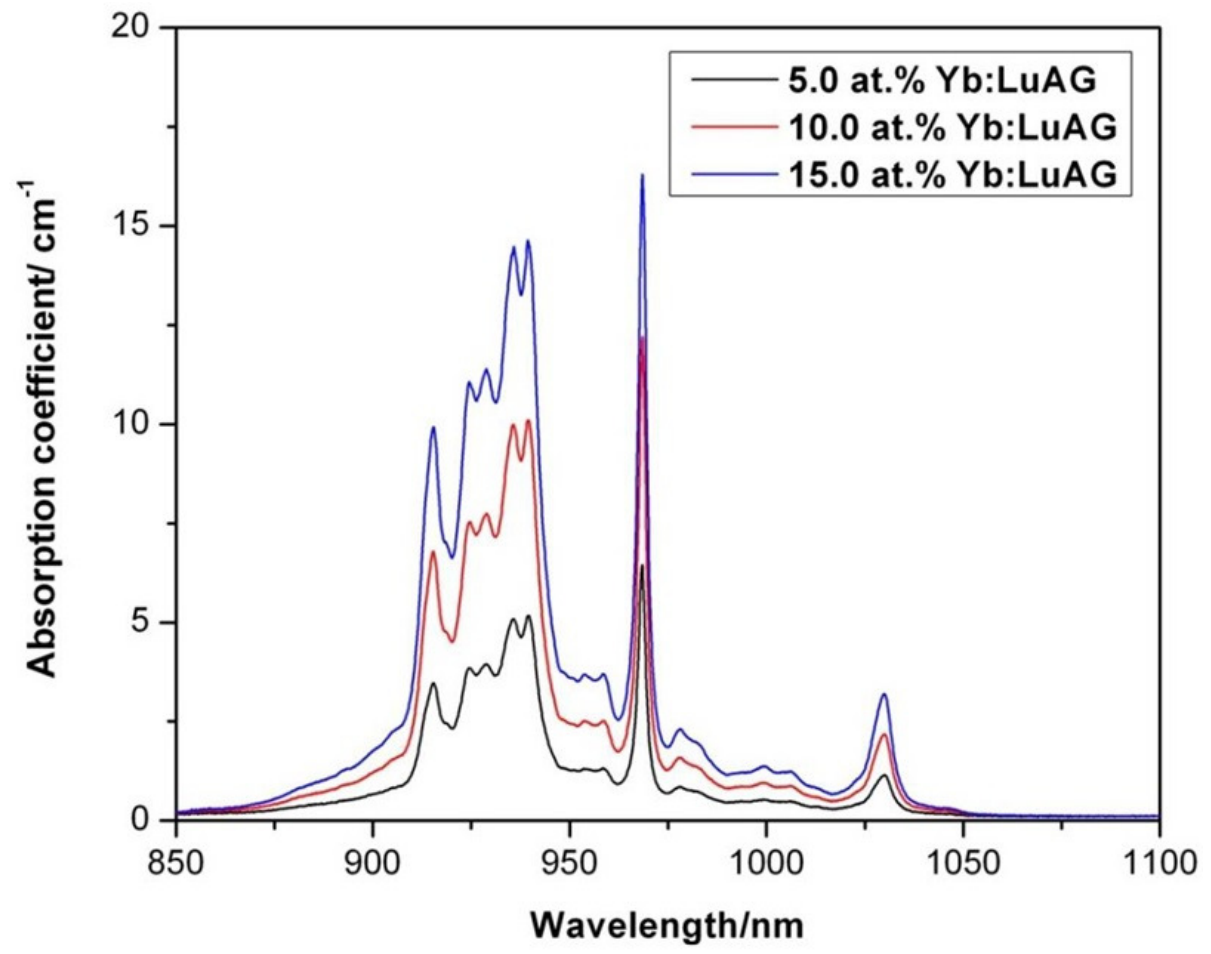

Figure 6.7 Absorption spectra of $\mathrm{Yb}: \mathrm{LuAG}$ ceramic samples with different $\mathrm{Yb}$ doping. 
Emission cross-section spectrum and fluorescent decay curve (inset) of the 5.0 at.\% $\mathrm{Yb}: \mathrm{LuAG}$ ceramic sample is shown in Figure 6.8. The main emission peak at the 1030 $\mathrm{nm}$ wavelength has a FWHM of $6.8 \mathrm{~nm}$. The emission cross-section at the peak is calculated to be $2.7 \times 10^{-20} \mathrm{~cm}^{2}$ by using the Füchtbauer-Ladenburg equation. The result is comparable with that of $\mathrm{Yb}: \mathrm{LuAG}$ single crystals. The value is about $30 \%$ higher than that of the Yb:YAG $\left(\sim 2.0 \times 10^{-20} \mathrm{~cm}^{2}\right)$. Laser gain media with a larger emission cross section will have a lower lasing threshold. The fluorescent lifetime of the doped ions in the sample was also measured, and a single exponential decay curve was observed. It gives a lifetime value of $0.96 \mathrm{~ms}$.

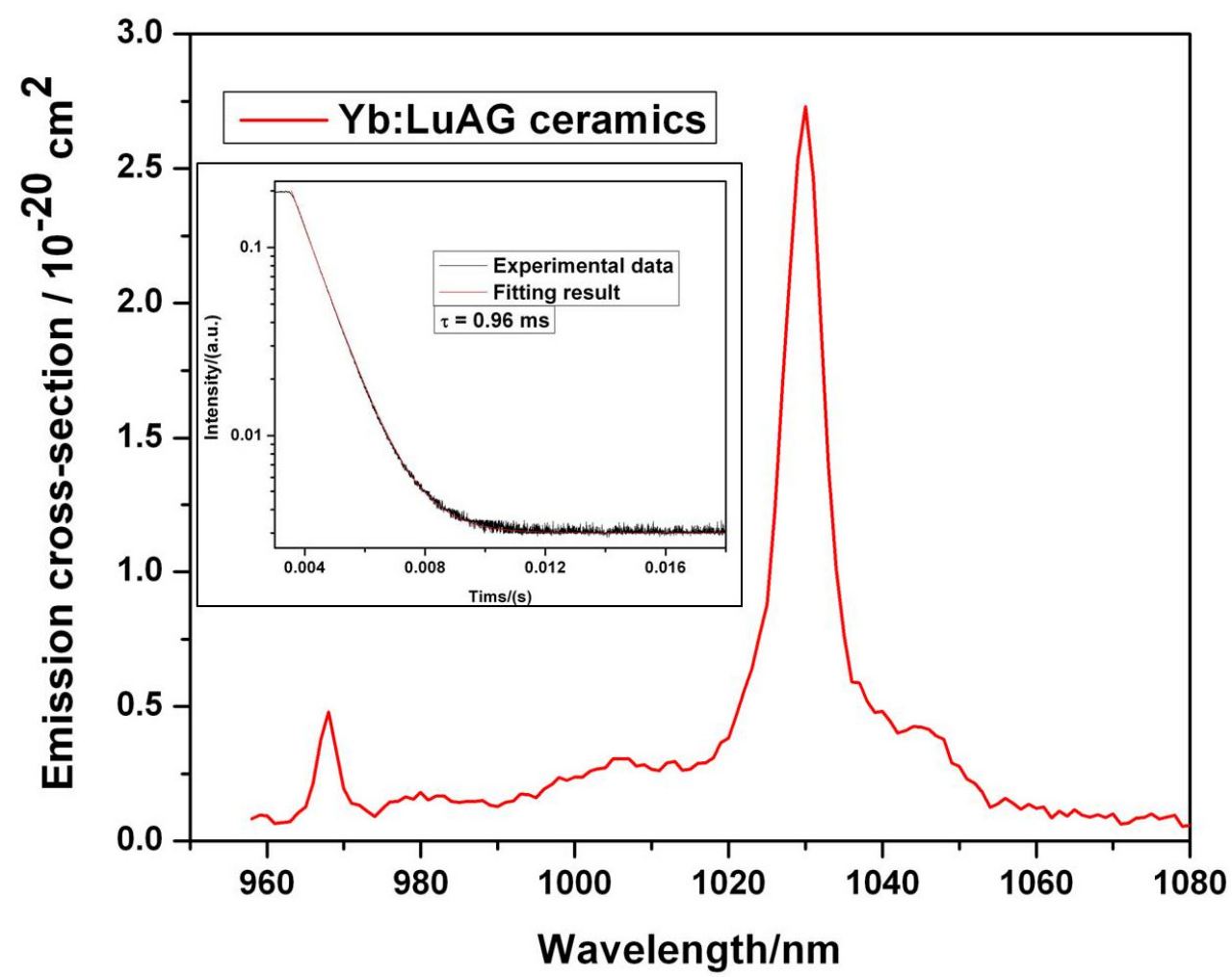

Figure 6.8 Emission cross-section and decay curve (inset) of the 5.0 at.\% Yb:LuAG ceramic sample.

The gain cross-section of the Yb:LuAG ceramics was then determined under different population inversion ratio $\beta$, which equals to $\mathrm{N}_{2} / \mathrm{N}_{0}$, where $\mathrm{N}_{2}$ is the inversion population number and $\mathrm{N}_{0}$ is the total population number. The gain cross-section $\sigma_{\mathrm{g}}(\lambda)$ value was 
calculated by Equation (4.5) and the calculated emission and absorption cross-section value of $\mathrm{Yb}: \mathrm{LuAG}$ ceramics. The gain cross-section of the $\mathrm{Yb}: \mathrm{LuAG}$ ceramics under different $\beta$ was plotted in Figure 6.9.

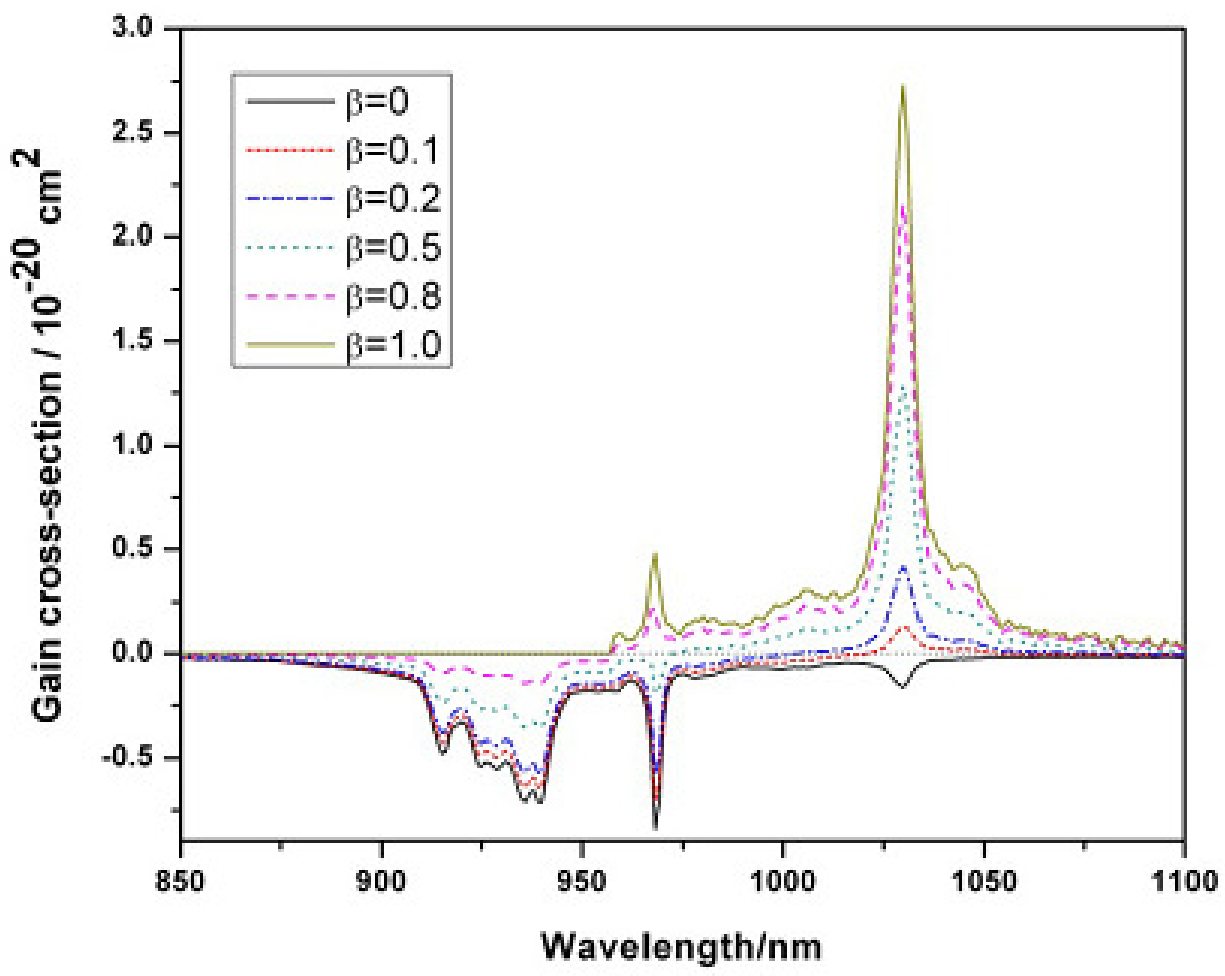

Figure 6.9 Gain cross-section of Yb:LuAG ceramics under different value of inversion ratio $\beta$.

\subsubsection{CW and mode-locked laser performance}

Laser performance of the 5.0 at $\% \mathrm{Yb}: \mathrm{LuAG}$ ceramic sample was investigated. The $\mathrm{CW}$ laser set up was similar to that for the $\mathrm{Yb}$ :YAG ceramics discussed in the Chapter 4 . The $\mathrm{Yb}$ :LuAG ceramic sample was cut into $3 \times 3 \times 3 \mathrm{~mm}^{3}$ cubic and both surfaces were high transmission coated at the pump and lasing wavelengths. CW laser performance result is shown in Figure 6.10. 


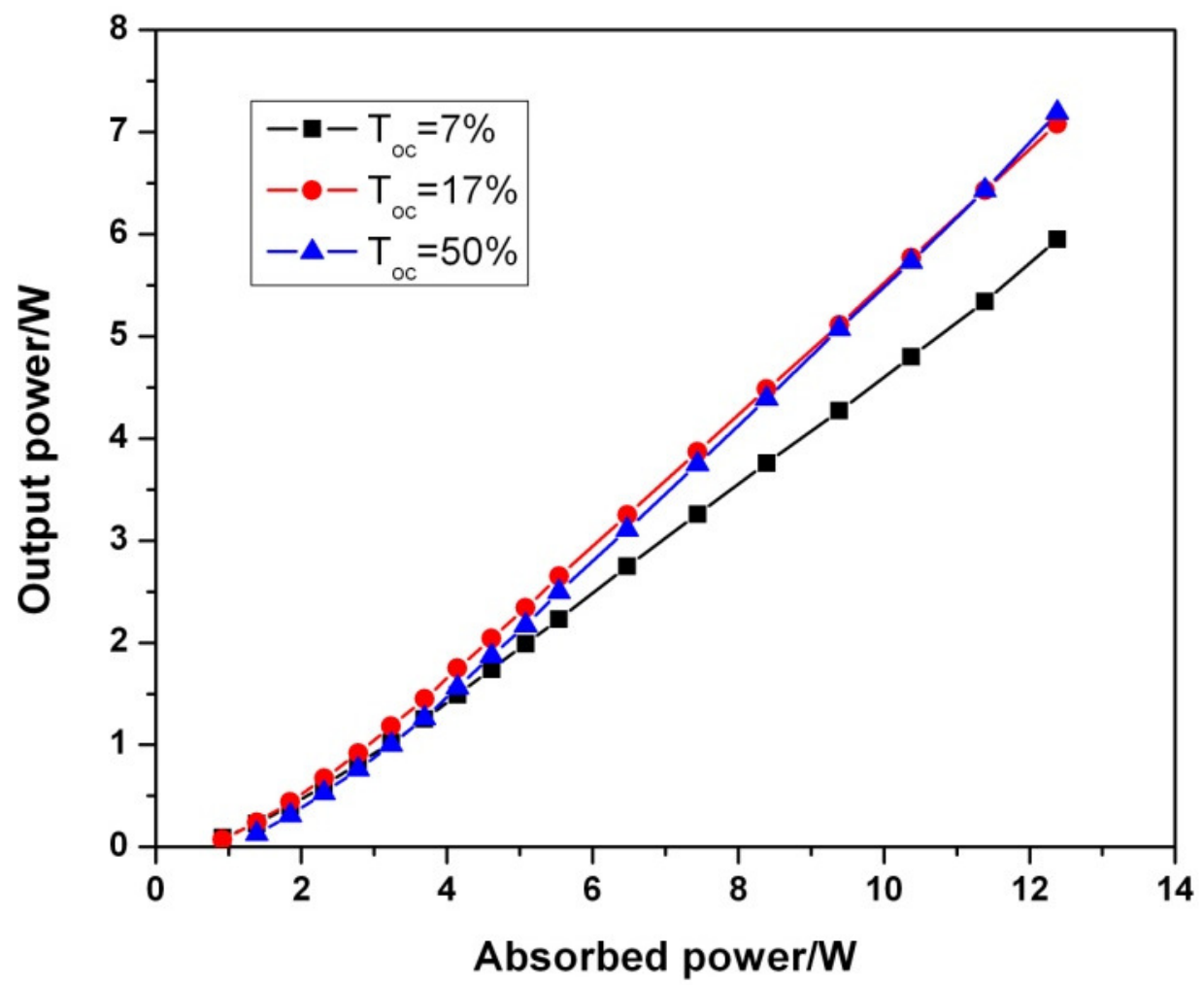

Figure 6.10 $\mathrm{CW}$ laser performance of the 5.0 at.\% Yb:LuAG ceramic sample under different output couplers.

CW laser operation at $1030 \mathrm{~nm}$ was successfully demonstrated with output couplers of different output couplings. Under an output coupler with Toc $=50 \%$, a maximum laser output power of $7.2 \mathrm{~W}$ was obtained at the absorbed pump power of $12.4 \mathrm{~W}$, corresponding to a slope efficiency of $65 \%$. The good result was also highlighted by laser focus world magazine [32].

Mode-locked laser performance was also investigated. Cavity layout for the mode-locked laser experiment is shown in Figure 6.11. The same fiber coupled $940 \mathrm{~nm}$ laser diode was used as pump source. For mode-locked laser operation, a semiconductor saturable absorbing mirror (SESAM BATOP GmbH) was used in the cavity. The SESAM has a modulation depth of $1 \%$ at $1030 \mathrm{~nm}$, with a relaxation time of $500 \mathrm{fs}$ and a non-saturable loss of $0.3 \%$. The output coupler has a transmittance of Toc=3.5\%. An SF10 prism pair was also inserted in the cavity to compensate the positive group delay dispersion. 
A fast detector (New Focus 1611, $1 \mathrm{GHz}$ bandwidth) together with a digital oscilloscope (Tektronix DPO 7104, $1 \mathrm{GHz}$ bandwidth) was employed to monitor the mode-locking pulse train. An optical spectrum analyzer (Ando AQ 6315B) was used to measure the optical spectrum and a commercial autocorrelator (APE GmbH, Pulsecheck) was used to measure the pulse width.

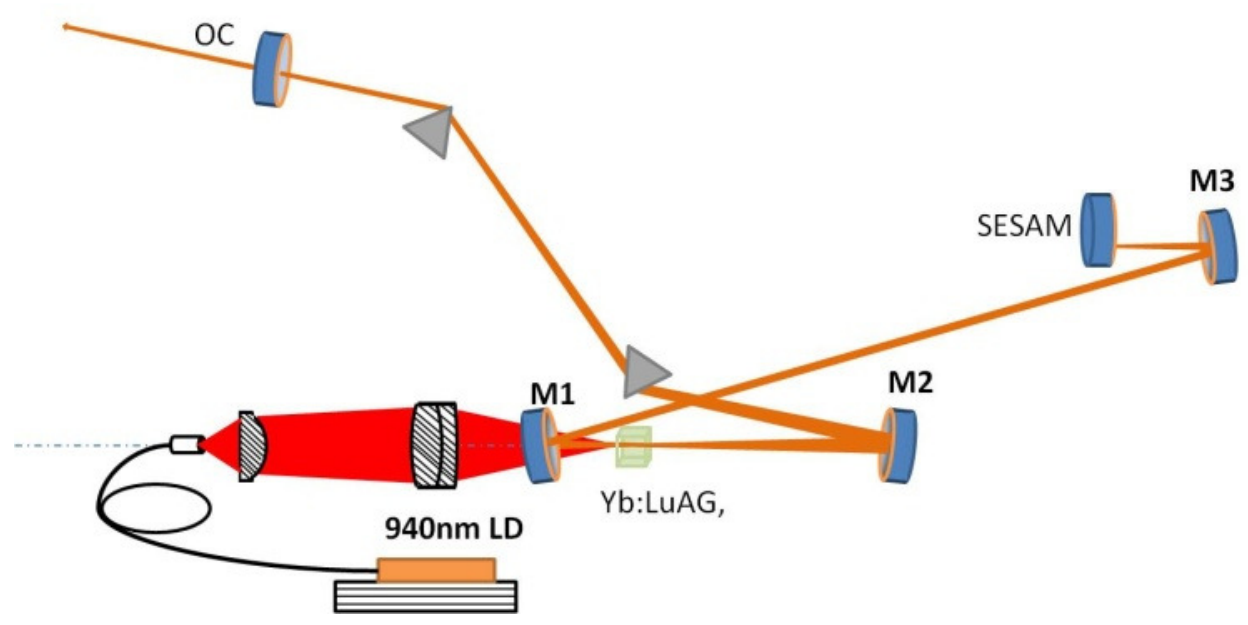

Figure 6.11 Schematic drawing of cavity design of passively mode-locked $\mathrm{Yb}: \mathrm{LuAG}$ ceramic laser.

It is observed that, under the incident pump power of $3.2 \mathrm{~W}$, stable mode locking was obtained with a $200 \mathrm{~mW}$ average output power. The reasons for the low output power of mode-locking operation mainly include the insertion of the prism pair and the relatively low transmittance of the output coupler. Threshold for the CW mode-locking is about 80 $\mathrm{mW}$ of output power, at pump power of $2 \mathrm{~W}$. When the pump power is increased, the output power increases as well. However multiple-pulse operation occurs.

Figure 6.12 shows the autocorrelation trace of mode-locked laser operation of the $\mathrm{Yb}: \mathrm{LuAG}$ ceramics, with a laser spectrum shown in the inset. The pulse repetition rate was $91 \mathrm{MHz}$. The pulse duration was measured to be $650 \mathrm{fs}$, if a sech ${ }^{2}$ pulse shape is assumed. It is shorter than that reported by Nakao et al.[33]. 


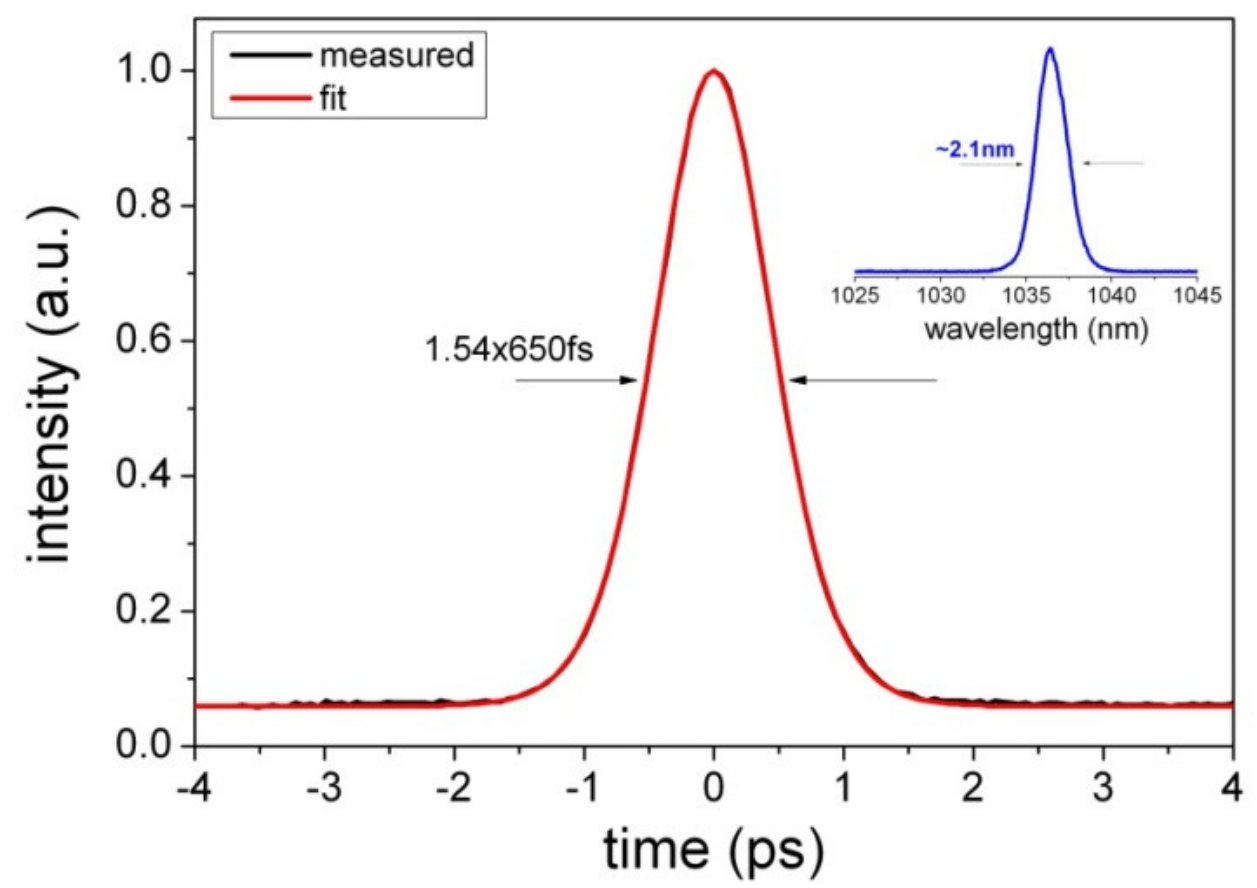

Figure 6.12 Autocorrelation trace of the mode-locked Yb:LuAG ceramics laser with laser spectrum in the inset.

Wavelength of the mode-locked laser pulse was centered at $1036.4 \mathrm{~nm}$ with a FWHM of $2.1 \mathrm{~nm}$, so the time bandwidth product is 0.38 , close to the transform limit of 0.315 . The wavelength shift of the mode-locking laser is believed to be caused by the strong reabsorption at the $1030 \mathrm{~nm}$ absorption peak, so that the mode-locked lasing wavelength shifts to longer wavelength.

\subsection{Conclusions}

In this chapter, by using solid-state reactive sintering method, the mixed garnet YLuAG and end compound LuAG ceramics with different $\mathrm{Yb}$ doping concentration were fabricated in-house, which was reported for the first time. The obtained samples showed relatively high optical transmittance, which is good enough for lab-scale laser experiment. $\mathrm{CW}$ and femtosecond mode-locked laser performance has also been achieved. With proper ceramic fabrication and laser engineering, there is still room for improvement. 
Since $\mathrm{Yb}: \mathrm{LuAG}$ ceramics have 5-10\% higher thermal conductivity and 30\% higher emission cross section than Yb:YAG ceramics, it will be an attractive gain medium for the high power laser applications, especially in thin disk laser design in which high doping and high thermal conductivity is desired .

\section{References}

[1] A. Giesen, H. Hügel, A. Voss, K. Wittig, U. Brauch, and H. Opower, Applied Physics B. 1994, 58, 365-372.

[2] T. Fan, IEEE Journal of Quantum Electronics. 1993, 29, 1457-1459.

[3] J. Marmo, H. Injeyan, H. Komine, S. McNaught, J. Machan, and J. Sollee, presented at SPIE LASE: Lasers and Applications in Science and Engineering. 2009.

[4] T. Y. Fan, T. Crow, and B. Hoden, presented at Aerospace/Defense Sensing and Controls. 1998.

[5] K. Beil, S. T. Fredrich-Thornton, F. Tellkamp, R. Peters, C. Kränkel, K. Petermann, and G. Huber, Optics Express. 2010, 18, 20712-20722.

[6] R. Gaumé, B. Viana, D. Vivien, J.-P. Roger, and D. Fournier, Applied Physics Letters. 2003, 83, 1355-1357.

[7] Y. Kuwano, K. Suda, N. Ishizawa, and T. Yamada, Journal of Crystal Growth. 2004, 260, 159-165.

[8] A. Petrosyan, G. Shirinyan, K. Ovanesyan, and A. Kuzanyan, Journal of Crystal Growth. 1981, 52, 556-560.

[9] P. Wu, and A. D. Pelton, Journal of Alloys and Compounds. 1992, 179, 259-287.

[10] A. Petrosyan, V. Popova, V. Gusarov, G. Shirinyan, C. Pedrini, and P. Lecoq, Journal of Crystal Growth. 2006, 293, 74-77.

[11] D. C. Brown, C. D. McMillen, C. Moore, J. W. Kolis, and V. Envid, Journal of Luminescence. 2014, 148, 26-32.

[12] X. Xu, X. Wang, J. Meng, Y. Cheng, D. Li, S. Cheng, F. Wu, Z. Zhao, and J. Xu, Laser Physics Letters. 2009, 6, 678-681.

[13] J. Di, X. Xu, J. Meng, D. Li, D. Zhou, F. Wu, and J. Xu, Laser Physics. 2011, 21, 844-846. 
[14] X. Chen, S. Zhao, J. Zhao, K. Yang, G. Li, D. Li, W. Qiao, T. Li, H. Zhang, and T. Feng, Optics \& Laser Technology. 2014, 64, 7-10.

[15] X. Xu, J. Di, W. Tan, J. Zhang, D. Tang, D. Li, D. Zhou, and J. Xu, Laser Physics Letters. 2012, 9, 406.

[16] W. Drozdowski, K. Brylew, M. Witkowski, A. Drewniak, Z. Masewicz, A. Wojtowicz, J. Kisielewski, and M. Świrkowicz, Optical Materials. 2016, 59, 107 114.

[17] M. Nikl, A. Yoshikawa, K. Kamada, K. Nejezchleb, C. Stanek, J. Mares, and K. Blazek, Progress in Crystal Growth and Characterization of Materials. 2013, 59, 47-72.

[18] T. Yanagida, Y. Fujimoto, Y. Yokota, K. Kamada, S. Yanagida, A. Yoshikawa, H. Yagi, and T. Yanagitani, Radiation Measurements. 2011, 46, 1503-1505.

[19] M. Sugiyama, Y. Fujimoto, T. Yanagida, Y. Yokota, J. Pejchal, Y. Furuya, H. Tanaka, and A. Yoshikawa, Optical Materials. 2011, 33, 905-908.

[20] K. Beil, S. T. Fredrich-Thornton, R. Peters, K. Petermann, and G. Huber, presented at Advanced Solid-State Photonics. 2009.

[21] D. Sumida, T. Y. Fan, and R. Hutcheson, presented at Advanced Solid State Lasers. 1995.

[22] A. Brenier, Y. Guyot, H. Canibano, G. Boulon, A. Ródenas, D. Jaque, A. Eganyan, and A. Petrosyan, Journal of the Optical Society of America B. 2006, 23, 676-683.

[23] J. Dong, K. Ueda, and A. A. Kaminskii, Optics letters. 2007, 32, 3266-3268.

[24] J. He, X. Liang, J. Li, H. Yu, X. Xu, Z. Zhao, J. Xu, and Z. Xu, Optics Express. 2009, 17, 11537-11542.

[25] J. Dong, K. Ueda, and A. Kaminskii, Laser Physics Letters. 2010, 7, 726-733.

[26] Y. Liao, D. Jiang, and J. Shi, Materials Letters. 2005, 59, 3724-3727.

[27] L. Pan, B. Jiang, J. Fan, P. Zhang, X. Mao, and L. Zhang, Optical Materials. 2016, In Press, http://dx.doi.org/10.1016/j.optmat.2016.06.020.

[28] C. Xu, D. Luo, J. Zhang, H. Yang, X. Qin, W. Tan, and D. Tang, Laser Physics Letters. 2012, 9, 30-34.

[29] M. Siebold, M. Loeser, F. Roeser, M. Seltmann, G. Harzendorf, I. Tsybin, S. Linke, S. Banerjee, P. Mason, and P. Phillips, Optics Express. 2012, 20, 21992-22000. 
[30] A. Pirri, M. Vannini, V. Babin, M. Nikl, and G. Toci, Laser Physics. 2013, 23, 095002.

[31] Y. Fu, J. Li, C. Wang, T. Xie, W. Li, L. Wu, and Y. Pan, Journal of Alloys and Compounds. 2016, 664, 595-601.

[32] J. Zhang, Laser Focus World, 2012, 2, 1421-1425.

[33] H. Nakao, A. Shirakawa, K.-i. Ueda, H. Yagi, and T. Yanagitani, Optics Express. 2012, 20, 15385-15391. 


\section{Chapter 7}

\section{Conclusions and Future Works}

In this chapter, a general discussion is presented by summarizing and concluding the results achieved throughout the thesis. By analyzing the current fabrication method in Yb doped garnet transparent laser ceramic fabrication, the potential improvement for futuristic research is also addressed. Exploration on obtaining new types of ceramic materials is carried out, with some preliminary results presented for fabricating high quality laser ceramics. 


\subsection{Conclusions}

This thesis focused on the experimental study of advanced ceramic fabrication and processing techniques, aiming to establish the most cost-effective and repeatable fabrication method for high quality laser ceramics. Exploration of new types of laser ceramics materials have been carried out. Solid state laser systems have been built for laser performance testing for the in-house fabricated ceramics samples. The main contributions and major results are summarized as follows:

(1) Using high purity $\mathrm{Yb}_{2} \mathrm{O}_{3}, \mathrm{Al}_{2} \mathrm{O}_{3}$ and $\mathrm{Y}_{2} \mathrm{O}_{3}$ commercial powders as the raw materials, high optical quality ytterbium-doped yttrium aluminum garnet (Yb:YAG) laser ceramics were obtained using advanced ceramics processing techniques. High energy ball milling process was used for raw powder processing. Dry press and cold isostatic pressing techniques were used to form green-body pellets. Vacuum sintering method was applied for samples densification. XRD and SEM results showed that as-fabricated samples had pure YAG phase and almost pore-free microstructure. Optical transmittance of the samples reached $83 \%$ at non-absorption wavelength range, which is very close to the theoretical transmittance value, indicating the sample's good optical quality. Continuous wave laser operation of samples was successfully achieved. Maximum laser output power of $6.2 \mathrm{~W}$ was obtained by using the 5.0 at.\% Yb:YAG ceramics, corresponding to a laser efficiency of $62.7 \%$.

(2) Based on the established fabrication method, a new type of ytterbium-doped gadolinium yttrium aluminum garnet (Yb:GdYAG) mixed garnet laser ceramics with good quality was fabricated. Continuous wave laser operation of the 5.0 at.\% Yb:GdYAG ceramic has been achieved at $4.27 \mathrm{~W}$ output power with $26.7 \%$ slope efficiency. Passive mode-locking properties of the ceramics were also firstly demonstrated with a semiconductor saturable absorbing mirror (SESAM). Stable mode-locked pulses with 4.4 picosecond pulse width and a maximum output power of $3.75 \mathrm{~W}$ at the wavelength of $1030.4 \mathrm{~nm}$ have been generated. Improvement in the optical quality of samples and laser slope efficiency could be possible in future. 
(3) High quality ytterbium-doped lutetium aluminum garnet (Yb:LuAG) ceramics samples were developed and reported for the first time. Emission cross-section of the $\mathrm{Yb}: \mathrm{LuAG}$ ceramics was calculated to be $2.7 \times 10^{-20} \mathrm{~cm}^{2}$. CW laser performance of the $\mathrm{Yb}: \mathrm{LuAG}$ ceramics was presented to have a $65 \%$ slope efficiency. Mode-locked laser operation of the sample was also demonstrated with 650 femtosecond pulse duration. Because of its high thermal conductivity and large emission cross-section, highly doped Yb:LuAG ceramics should have a huge potential application for generating ultra-short pulses in thin-disk lasers.

\subsection{Recommendations for future work}

According to the current results, future works are proposed as follows. Physical characteristics, such as dielectric properties, defect chemistry of the optical materials and origin of the excellent optical properties of YAG-based ceramics, should be further studied. Theoretical aspects on physics and chemistry of laser ceramic materials should be clarified. In particular, some specific works are briefly described with the support of preliminary results in the following sections.

\subsubsection{Exploration for new type of ytterbium co-doped garnet laser ceramics}

Besides single doping, it is not unusual to co-dope two more multiple rare earth ions in the gain medium, to achieve the unique feature in spectroscopic properties [1-4]. For example, ytterbium ion can be co-doped together with erbium (Er) ion $[5,6]$. Typically, ytterbium ions can directly absorb the pump radiation between $900 \mathrm{~nm}$ to $1000 \mathrm{~nm}$ wavelength range and transfer the energy to erbium ions through the direct energy transfer (DT) from the ${ }^{2} \mathrm{~F}_{5 / 2}$ level of $\mathrm{Yb}^{3+}$ ion to the ${ }^{4} \mathrm{I}_{11 / 2}$ level of $\mathrm{Er}^{3+}$ ion. It is a way to increase the output efficiency by improving the absorption to the pump sources. So the $\mathrm{Er}, \mathrm{Yb}$ co-doped optical materials have potential improved applications in the 1.5-1.6 $\mu \mathrm{m}$ eye-safe wavelength ranges. The Er, $\mathrm{Yb}$ co-dpoed $\mathrm{YAG}$ single crystal have been demonstrated firstly by Schweizer et al. early in 1995 [7]. Zhou et al. have first studied the optical properties of the Er, Yb co-doped YAG transparent ceramics [8]. By using the 
previously established Yb:YAG ceramics fabrication method, we have also successfully fabricated Er,Yb:YAG ceramics with different doping concentration. Figure 7.1 shows the transmittance curve of Er,Yb co-doped YAG ceramics with photo of samples in the insert. CW laser was realized at $1.6 \mu \mathrm{m}$ wavelength of the particular sample [9]. Further improvement of the sample optical quality as well as doping concentration could be carried out in order to achieve higher laser output power and efficiency.

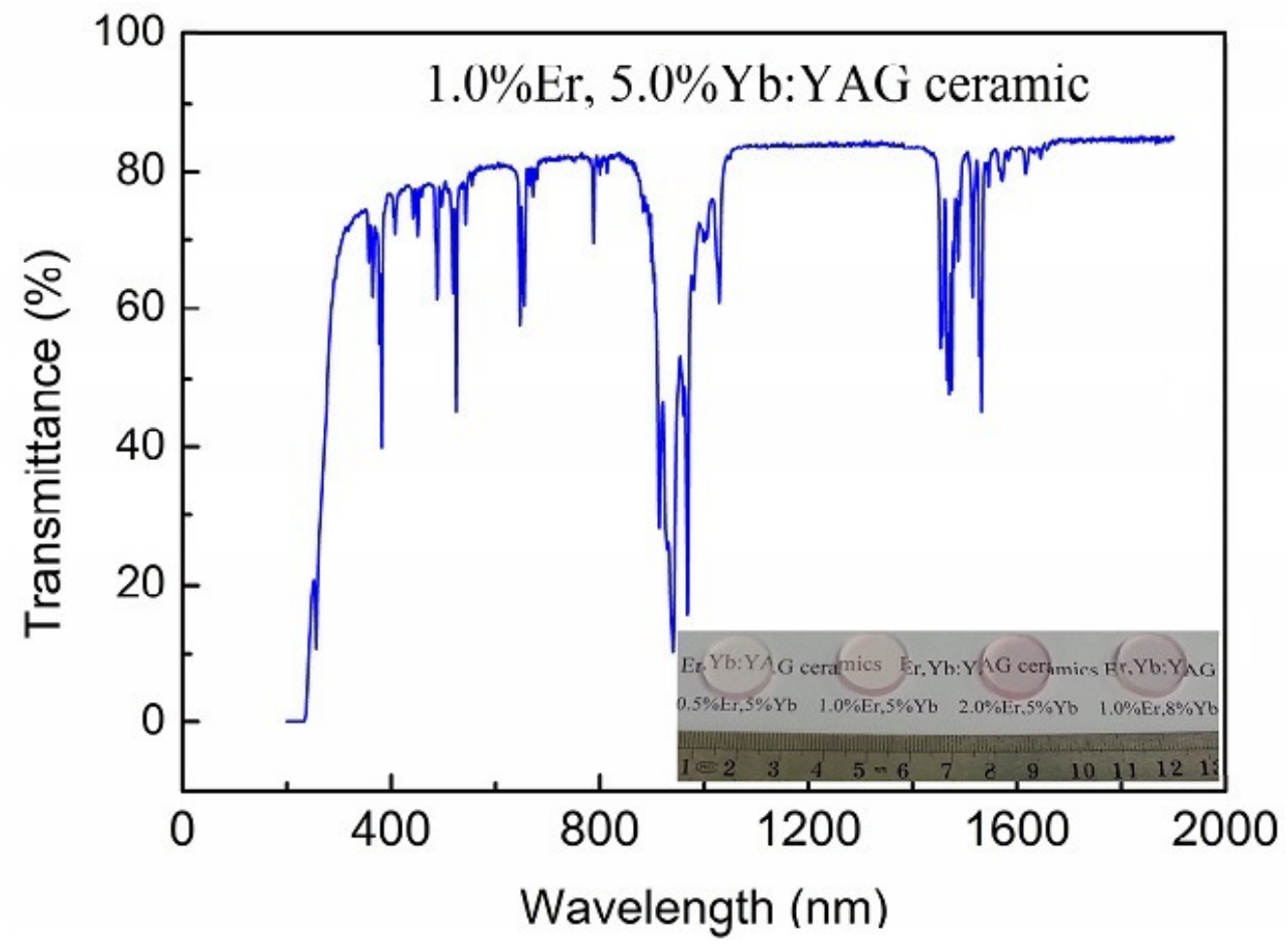

Figure 7.1 Transmittance curve of $\mathrm{Er}, \mathrm{Yb}$ co-doped YAG ceramics with photo of samples in the insert.

\subsubsection{Composite structures laser ceramics}

The conventional laser gain medium consists of only one simple composition and a homogeneous and uniform design in one bulk piece. With the development of laser technology, this kind of conventional design may not be able to meet the requirements for new applications and functionality. So the composite laser material was proposed by optically bonding two or more parts of different material compositions together [10]. The 
conventional bonding technology needs to have precise optical polishing of the bonding surface and mostly limited to only flat surfaces. So the bonding strength at the interface is not very high. With the help of advanced ceramic technology these limitations may be solved. Different material composition can be determined at the green-body forming stage. For example, powders of different material dopings can be stacked together to create multilayer structure, or cylindrical core-cladding structure and then compacted together to form a single piece of green-body. Followed by sintering process, then the composite structures ceramic can be obtained [11-13].

Figure 7.2 shows the in-house fabricated composite Yb:YAG laser ceramics. The sample on the left shows outer ring is made of Yb:YAG (un-annealed) showing the green color while inner circle is un-dpoed YAG (colorless). The middle sample is vise versa. The sample on the right is after annealing process. Both doped and undpoed parts appear colorless and the boundary is not obviously visible. The specific design parameters such as the doping element, doping concentration, size and shape will depends on the requirement for specific laser system's applications and design.

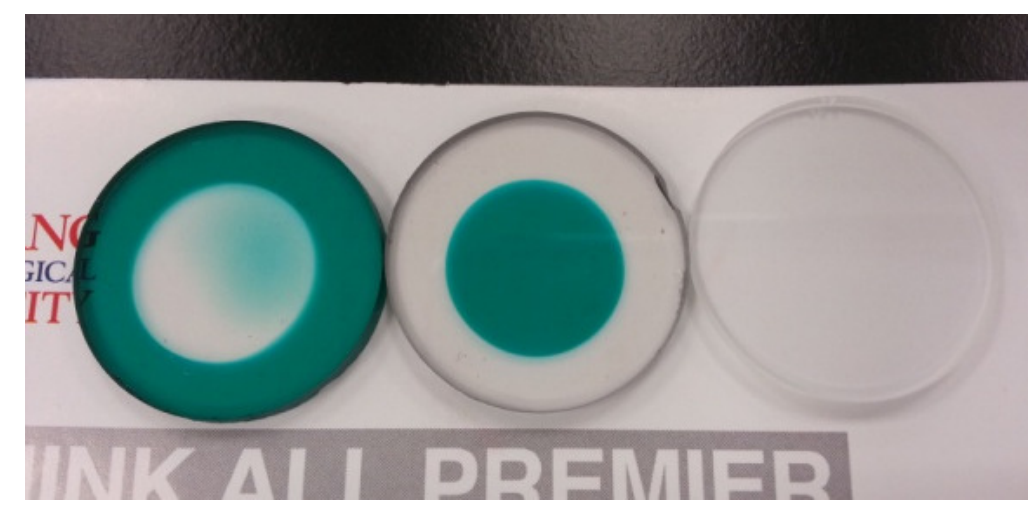

Figure 7.2 Photo of composite Yb:YAG laser ceramics.

\subsubsection{Sesquioxide ceramics as laser host material}

Rare-earth doped sesquioxide crystals, such as yttria $\left(\mathrm{Y}_{2} \mathrm{O}_{3}\right)$ [14], lutetia $\left(\mathrm{Lu}_{2} \mathrm{O}_{3}\right)$ [15] and Scandia $\left(\mathrm{Sc}_{2} \mathrm{O}_{3}\right)$ [16], are also promising high power and ultra short pulse solid state laser 
gain media due to their broad gain bandwidth, small quantum defect, strong crystal field effects, excellent thermo-mechanical properties and low phonon energies [17, 18]. Table 7.1 lists some of the properties for sesquioxides compared with YAG [19]. However the growth of large crystals from the melt is a very difficult and high cost job due to their high melting point of more than $2400^{\circ} \mathrm{C}$. But transparent sesquioxide ceramics of could be fabricated with large size sample at relatively lower sintering temperature by using proper sintering additives and sintering method. Good quality ytterbium doped yttria $\left(\mathrm{Yb}: \mathrm{Y}_{2} \mathrm{O}_{3}\right)$ ceramics have been obtained using commercial powders as raw material, and go through ball milling and vacuum sintering method [20-22]. Optimization in sintering additives and sintering temperature to improve samples quality is also possible in future.

Table 7.1 Properties of the sesquioxides compared with YAG.

\begin{tabular}{l|l|l|l|l}
\hline & $\mathrm{Y}_{2} \mathrm{O}_{3}$ & $\mathrm{Lu}_{2} \mathrm{O}_{3}$ & $\mathrm{Sc}_{2} \mathrm{O}_{3}$ & $\mathrm{YAG}$ \\
\hline Melting point $\left({ }^{\circ} \mathrm{C}\right)$ & $\sim 2430$ & $\sim 2450$ & $\sim 2430$ & 1930 \\
\hline Crystal Structure & cubic & cubic & cubic & cubic \\
\hline Lattice constant $(\AA)$ & 10.603 & 10.391 & 9.844 & 12.00 \\
\hline Coordination numbers & 6 & 6 & 6 & $8 ; 6 ; 4$ \\
\hline $\begin{array}{l}\text { Thermal conductivity un-doped }(\text { at } \\
\left.30^{\circ} \mathrm{C}\right)(\mathrm{W} / \mathrm{mK})\end{array}$ & 13.6 & 12.5 & 16.5 & 11.0 \\
\hline $\begin{array}{l}\text { Doped with 3\% } \mathrm{Yb}\left(\mathrm{at} 30^{\circ} \mathrm{C}\right) \\
\text { (W/mK) }\end{array}$ & 7.7 & 11.0 & 6.6 & 6.8 \\
\hline $\begin{array}{l}\text { Eff. phonon energy for dopants }(\mathrm{cm}- \\
\text { 1) }\end{array}$ & 430 & $<430$ & $<625$ & 700 \\
\hline Mohs hardness & 6.8 & $\sim 7$ & $<6.8$ & 8.5 \\
\hline
\end{tabular}

\subsubsection{Residual pore removal and ceramics densification study}

Although various ytterbium doped laser ceramics with high optical transmittance have been fabricated and their laser performances have been successfully demonstrated, there are still random defect spots that can be found in the bulk interior of the samples, which could be visible while they are shined with strong light source. These light reflection 
spots are suspected to be residual pores or impurities. In the future work, these defect spots will be further monitored and investigated by using optical microscope and SEM analysis. In low power laser experiment, the sample size needed is only about several millimeters, so the defect spots can be avoided through sample cutting or adjusting laser spot in a defect-free area. However, for large scale high power lasers, these defect spots will cause large amount of light scattering which will reduce the laser efficiency or even damage the laser system. Therefore the defects must be truly eliminated, which is one of the hallenges of the further study. As mentioned previously, controlling the residual pores and defects in the ceramics body is one of the most important and challenging issues in the fabrication of laser ceramics. Careful environmental control during raw powder preparation and shaping is quite important for control foreign contamination. And the most important part is the sintering process, during which the ceramics densification and pore elimination process will occur. During sintering process, sintering additives are used to achieve high optical quality ceramics. Previous research showed that silica was a very effective sintering aid for YAG ceramics, since it was believed to facilitate the formation of liquid phase, so as to enhance the densification process [23]. The future work will be focused on the effect of sintering temperature and amount of sintering additive on microstructure evolution, as well as their effect on the ceramic's optical properties. The interrelations among density, grain size, pore size (type) will be studied as functions of sintering temperature and the content of sintering aid. These will allow establishing sintering trajectories and sintering maps, as guides for fabrication of transparent ceramics with desired optical performance.

New sintering method for fabrication of transparent laser ceramics will also be explored. Hot isostatic press (HIP) sintering has been verified to be an effective sintering technique to reduce porosity and achieve full densification of transparent ceramics without much grain growth, as the applied high pressure during the final-stage sintering can provide strong driving force for densification [24]. 


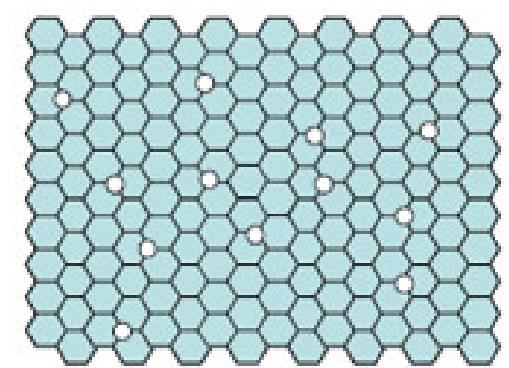

Low temperature pre-sintered

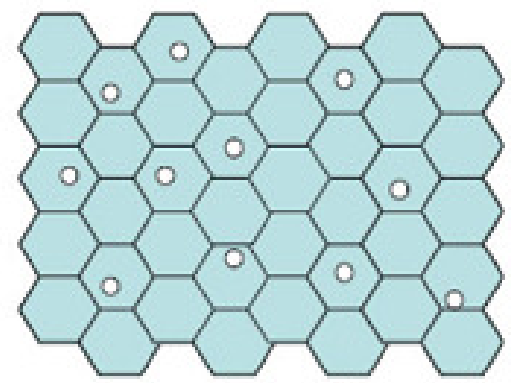

High temperature pre-sintered

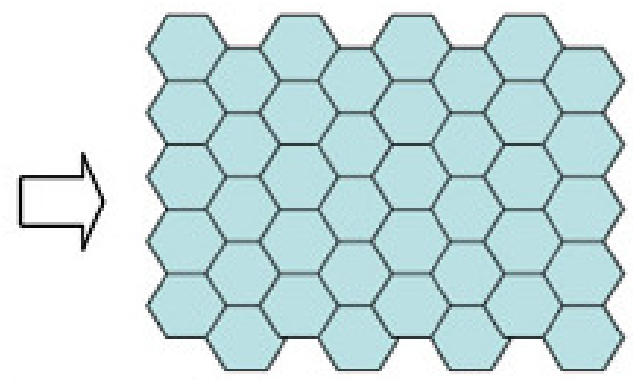

Hot isostatic pressed

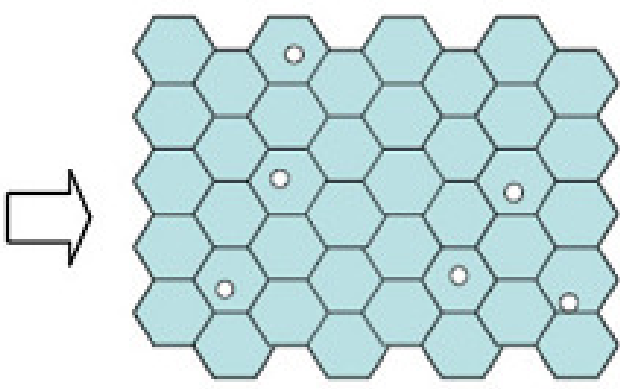

Hot isostatic pressed

Figure 7.3 Model for the pore elimination of hot isostatic pressing sintering.

Figure 7.3 shows the model for the pore elimination of hot isostatic pressing sintering [25]. Small intergranular pores will formed at the low pre-sintering temperatures, and they are easily expelled by the following hot isostatic pressing sintering. If the presintering temperature is relatively high, then intragranular pores will form and trapped within the grains by the grain growth. And they will remain as residual pores even when hot isostatic pressing sintering is followed. So the pre-sintering temperature will still be optimizes in the future experiment in order to obtain high quality transparent laser ceramics.

\section{References}

[1] G. Kumar, E. De la Rosa-Cruz, K. Ueda, A. Martınez, and O. Barbosa-Garcia, Optical Materials. 2003, 22, 201-213.

[2] R. Scheps, Progress in Quantum Electronics 1996, 20, 271-358. 
[3] H. Desirena, E. De la Rosa, L. Diaz-Torres, and G. Kumar, Optical materials 2006, $28,560-568$.

[4] J. Ueda, and S. Tanabe, presented at Conference on Lasers and ElectroOptics/Pacific Rim. 2009.

[5] Y. Kishi, S. Tanabe, S. Tochino, and G. Pezzotti, Journal of the American Ceramic Society. 2005, 88, 3423-3426.

[6] V. Tikhomirov, V. Rodríguez, J. Méndez-Ramos, J. Del-Castillo, D. Kirilenko, G. Van Tendeloo, and V. Moshchalkov, Solar Energy Materials and Solar Cells. 2012, 100, 209-215.

[7] T. Schweizer, T. Jensen, E. Heumann, and G. Huber, Optics communications. 1995, $118,557-561$.

[8] J. Zhou, W. Zhang, T. Huang, L. Wang, J. Li, W. Liu, B. Jiang, Y. Pan, and J. Guo, Ceramics International. 2011, 37, 513-519.

[9] H. Zhu, D. Tang, Y. Duan, D. Luo, and J. Zhang, Optics express. 2013, 21, 2695526961.

[10] M. Tsunekane, N. Taguchi, T. Kasamatsu, and H. Inaba, IEEE Journal of Selected Topics in Quantum Electronics. 1997, 3, 9-18.

[11] J. Dong, A. Shirakawa, K. Ueda, H. Yagi, T. Yanagitani, and A. Kaminskii, Applied Physics Letters. 2007, 90, 191106.

[12] H. Yagi, K. Takachi, K. Ueda, Y. Yamasaki, T. Yanagitani, and A. Kaminskii, Laser Physics. 2005, 15, 1338-1344.

[13] W. Liu, Y. Zeng, J. Li, Y. Shen, Y. Bo, N. Zong, P. Wang, Y. Xu, J. Xu, and D. Cui, Journal of Alloys and Compounds. 2012, 527, 66-70.

[14] J. Lu, K. Takaichi, T. Uematsu, A. Shirakawa, M. Musha, K. Ueda, H. Yagi, T. Yanagitani, and A. Kaminskii, Japanese Journal of Applied Physics. 2002, 41, L1373.

[15] K. Takaichi, H. Yagi, A. Shirakawa, K. Ueda, S. Hosokawa, T. Yanagitani, and A. Kaminskii, Physica Status Solidi (a). 2005, 202, R1-R3.

[16] J. Lu, J. Bisson, K. Takaichi, T. Uematsu, A. Shirakawa, M. Musha, K. Ueda, H. Yagi, T. Yanagitani, and A. Kaminskii, Applied Physics Letters. 2003, 83, 1101. 
[17] K. Petermann, G. Huber, L. Fornasiero, S. Kuch, E. Mix, V. Peters, and S. Basun, Journal of Luminescence. 2000, 87, 973-975.

[18] K. Petermann, L. Fornasiero, E. Mix, and V. Peters, Optical Materials. 2002, 19, $67-71$.

[19] V. Peters, A. Bolz, K. Petermann, and G. Huber, Journal of Crystal Growth 2002, 237, 879-883.

[20] K. Ning, J. Wang, D. Luo, J. Ma, J. Zhang, Z. L. Dong, L. B. Kong, and D. Y. Tang, Optical Materials. 2015, 50, 21-24.

[21] K. Ning, J. Wang, D. Luo, Z. L. Dong, L. B. Kong, and D. Y. Tang, Journal of Alloys and Compounds. 2016.

[22] K. Ning, J. Wang, D. Luo, J. Zhang, Z. L. Dong, L. B. Kong, and D. Y. Tang, Journal of the European Ceramic Society. 2016, 36, 253-256.

[23] H. Van Dijk, Materials Research Bulletin. 1984, 19, 1669-1674.

[24] J. Wang, J. Ma, J. Zhang, P. Liu, D. Luo, D. Yin, D. Tang, and L. B. Kong, Optical Materials. 2016.

[25] K. Tsukuma, I. Yamashita, and T. Kusunose, Journal of the American Ceramic Society. 2008, 91, 813-818. 


\section{Publication List}

[1] D.W. Luo, C.W. Xu, J. Zhang, X.P. Qin, H. Yang, W.D. Tan, Z.H. Cong, and D.Y. Tang, "Diode pumped and mode-locked Yb:GdYAG ceramic lasers", Laser Physics Letters, 8 (2011) 719-722.

[2] D. Luo, J. Zhang, C. Xu, X. Qin, D. Tang, and J. Ma, "Fabrication and laser properties of transparent Yb: YAG ceramics”, Optical Materials, 34 (2012) 936-939.

[3] D. Luo, J. Zhang, C. Xu, H. Yang, H. Lin, H. Zhu, and D. Tang, "Yb: LuAG laser ceramics: a promising high power laser gain medium", Optical Materials Express, 2 (2012) 1425-1431.

[4] D.W. Luo, J. Zhang, C.W. Xu, H. Yang, X.P. Qin, J. Ma, and D.Y. Tang, "Fabrication and spectroscopic properties of transparent Yb:YAG laser ceramics", Solid State Phenomena, 185 (2012) 44-47.

[5] D. Luo, J. Zhang, C. Xu, H. Lin, H. Yang, H. Zhu, G. Shao, D. Tang, and L. Kong, "Mode-locked Yb:LuAG ceramics laser", Physica Satus Solidi (c), 10 (2013) 967-968.

[6] C. Xu, D. Luo, J. Zhang, H. Yang, X. Qin, W. Tan, and D. Tang, "Diode pumped highly efficient $\mathrm{Yb}: \mathrm{Lu}_{3} \mathrm{Al}_{5} \mathrm{O}_{12}$ ceramic laser", Laser Physics Letters, 9 (2012) 30-34.

[7] R. Zheng, D. Luo, Y. Yuan, Z. Wang, Y. Zhang, W. Wei, L.B. Kong, and D. Tang, "Dy ${ }^{3+} / \mathrm{Ce}^{3+}$ codoped YAG transparent ceramics for single-composition tunable white-light phosphor", Journal of the American Ceramic Society, 98 (2015) 3231-3235.

[8] S. Wang, J. Zhang, D. Luo, F. Gu, D. Tang, Z. Dong, G. Tan, W. Que, T. Zhang, and S. Li, "Transparent ceramics: processing, materials and applications", Progress in Solid State Chemistry, 41 (2013) 20-54.

[9] H. Yang, L. Zhang, D. Luo, X. Qiao, J. Zhang, T. Zhao, D. Shen, and D. Tang, "Optical properties of Ho:YAG and Ho:LuAG polycrystalline transparent ceramics". Optical Materials Express, 5 (2015) 142-148. 
[10] K. Ning, J. Wang, D. Luo, J. Ma, J. Zhang, Z.L. Dong, L.B. Kong, and D.Y. Tang, "Fabrication and characterization of highly transparent $\mathrm{Yb}: \mathrm{Y}_{2} \mathrm{O}_{3}$ ceramics", Optical Materials, 50 (2015) 21-24.

[11] J. Wang, J. Zhang, D. Luo, H. Yang, D. Tang, and L.B. Kong, "Densification and microstructural evolution of yttria transparent ceramics: the effect of ball milling conditions", Journal of the European Ceramic Society, 35 (2015) 1011-1019.

[12] K. Ning, J. Wang, D. Luo, J. Zhang, Z.L. Dong, L.B. Kong, and D. Y. Tang, "New double-sintering aid for fabrication of highly transparent ytterbiumdoped yttria ceramics", Journal of the European Ceramic Society, 36 (2016) 253-256.

[13] K. Ning, J. Wang, D. Luo, Z.L. Dong, L.B. Kong, and D.Y. Tang, "Lowlevel sintering aids for highly transparent $\mathrm{Yb}: \mathrm{Y}_{2} \mathrm{O}_{3}$ ceramics", Journal of Alloys and Compounds, 695 (2017) 1414-1419.

[14] X. Qin, H. Yang, G. Zhou, D. Luo, Y. Yang, J. Zhang, S. Wang, J. Ma, and D. Tang, "Fabrication and properties of highly transparent Er:YAG ceramics", Optical Materials, 34 (2012) 973-976.

[15] H. Zhu, D. Tang, Y. Duan, D. Luo, and J. Zhang, "Laser operation of diodepumped Er,Yb co-doped YAG ceramics at $1.6 \mu \mathrm{m}$ ", Optics express, 21 (2013) 26955-26961. 